\title{
WestVirginiaUniversity
}

THE RESEARCH REPOSITORY @ WVU

Graduate Theses, Dissertations, and Problem Reports

2009

\section{Image analysis of circulating fluidized bed hydrodynamics}

David Kent Casleton

West Virginia University

Follow this and additional works at: https://researchrepository.wvu.edu/etd

\section{Recommended Citation}

Casleton, David Kent, "Image analysis of circulating fluidized bed hydrodynamics" (2009). Graduate Theses, Dissertations, and Problem Reports. 2748.

https://researchrepository.wvu.edu/etd/2748

This Thesis is protected by copyright and/or related rights. It has been brought to you by the The Research Repository @ WVU with permission from the rights-holder(s). You are free to use this Thesis in any way that is permitted by the copyright and related rights legislation that applies to your use. For other uses you must obtain permission from the rights-holder(s) directly, unless additional rights are indicated by a Creative Commons license in the record and/ or on the work itself. This Thesis has been accepted for inclusion in WVU Graduate Theses, Dissertations, and Problem Reports collection by an authorized administrator of The Research Repository @ WVU. For more information, please contact researchrepository@mail.wvu.edu. 


\title{
Image Analysis of Circulating Fluidized Bed Hydrodynamics
}

\author{
by \\ David Kent Casleton \\ Thesis submitted to the \\ College of Engineering and Mineral Resources \\ at West Virginia University \\ in partial fulfillment of the requirements \\ for the degree of \\ Master of Science \\ in \\ Electrical Engineering \\ Arun Ross, PhD, Chair \\ Lawrence Shadle, $\mathrm{PhD}$ \\ Xin $\mathrm{Li}, \mathrm{PhD}$
}

Lane Department of Electrical Engineering and Computer Science

\author{
Morgantown, West Virginia \\ 2009
}

Keywords: image processing, particle segmentation, particle tracking

Copyright 2009 David Kent Casleton 


\author{
Abstract \\ Image Analysis of Circulating Fluidized Bed Hydrodynamics \\ by \\ David Kent Casleton \\ Master of Science in Electrical Engineering \\ West Virginia University \\ Arun Ross, PhD, Chair
}

The goal of this thesis is to design methods to estimate the local concentration and velocity of particles observed in digital videos of the inner wall of a circulating fluidized bed (CFB) riser. Understanding the dynamics of these rapidly moving particles will allow researchers to design cleaner and more efficient CFB facilities. However, the seemingly random motion exhibited by the particles in three dimensions, coupled with the varying image quality, make it difficult to extract the required information from the images. Given a video sequence, a method for detecting particles and tracking their spatial location is developed. By exploiting the presence of specular reflections, individual particles are first identified along the focal plane by an image filter specifically designed for this purpose. Once the particle locations are known, a local optical flow model is used to approximate the motion field across two images in order to track particles from one frame of the sequence to another. An evaluation of the proposed method indicates its potential to estimate particle count, location, concentration and velocity in an efficient and reliable manner. The method is fully automated and is expected to be an important analysis tool for researchers with the National Energy Technology Laboratory, part of the national laboratory system of the Department of Energy. 


\section{Acknowledgments}

This research was made possible by the experimental data, technical council, and project funding provided by the Computational Sciences Division within the National Energy Technology Laboratory (NETL), part of the national laboratory system of the Department of Energy (DOE). 
This page is intentionally blank. 


\section{Contents}

List of Figures $\quad$ vii

1 Introduction $\quad 1$

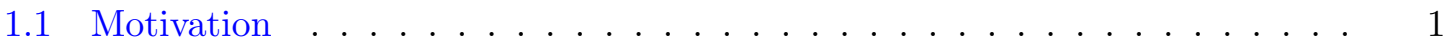

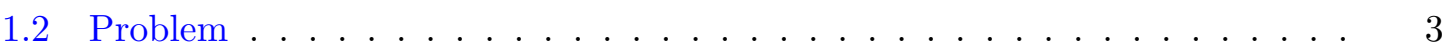

1.2.1 Internal Visualization . . . . . . . . . . . . . . 3

1.2 .2 External Visualization . . . . . . . . . . . . . . . 7

1.3 Project Goals . . . . . . . . . . . . . . . . . . . . . . . 11

1.4 Proposed Solution . . . . . . . . . . . . . . . . . . . . 11

1.4 .1 The Approach . . . . . . . . . . . . . . . . . 11

1.4 .2 Functional Requirements . . . . . . . . . . . . . . . . 12

1.4 .3 Advantages . . . . . . . . . . . . . . . . . . 13

2 Image Preprocessing $\quad 15$

2.1 Contrast Enhancement . . . . . . . . . . . . . . . . . . . 15

2.1.1 Contrast Stretching . . . . . . . . . . . . . . . . 16

2.1 .2 Histogram Equalization . . . . . . . . . . . . . . . . . . 19

2.1.3 Local Histogram Equalization . . . . . . . . . . . . . . . . . 21

2.2 Eliminating Non-uniform Background Illumination . . . . . . . . . . . . . 22

2.2.1 Top-hat Transformation . . . . . . . . . . . . . . . . 23

2.2 .2 Temporal Mean . . . . . . . . . . . . . . . . . . . 26

2.3 Specular Highlight Suppression . . . . . . . . . . . . . . . . . 28

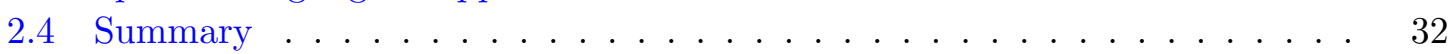

3 Particle Detection $\quad 35$

3.1 Introduction . . . . . . . . . . . . . . . . . . 35

3.2 Evaluation Method . . . . . . . . . . . . . . . . . 37

3.3 Classification Techniques . . . . . . . . . . . . . . . . . . 40

3.3.1 Intensity-based Classification . . . . . . . . . . . . . 40

3.3.2 Gradient-based Classification . . . . . . . . . . . . . 50

3.3.3 Learning-based Classification . . . . . . . . . . . . . . 58

3.4 Direct Identification by Specular Highlights . . . . . . . . . . . . . . 66

3.5 Estimated Solids Concentration . . . . . . . . . . . . . . . . 68

3.6 Summary . . . . . . . . . . . . . . . . . . . 73 
4 Particle Tracking $\quad \mathbf{7 5}$

4.1 Optical Flow . . . . . . . . . . . . . . . . . . 75

4.1.1 Lucas-Kanade Feature Tracker . . . . . . . . . . . . . . . . 76

4.1.2 Image Pyramid Implementation . . . . . . . . . . . . . . . . . . . . 77

4.2 Tracking Specular Highlights . . . . . . . . . . . . . . . . . . . . 79

4.3 Results . . . . . . . . . . . . . . . . . . . 80

4.4 Estimated Particle Velocities . . . . . . . . . . . . . . . . 83

4.5 Summary . . . . . . . . . . . . . . . . . . 83

5 Future Work $\quad \mathbf{8 5}$

5.1 Image Preprocessing . . . . . . . . . . . . . . . . . . . . . . 85

5.2 Particle Detection . . . . . . . . . . . . . . . . . . 85

5.3 Particle Tracking . . . . . . . . . . . . . . . 86

$\begin{array}{lc}\text { References } & 87\end{array}$ 


\section{List of Figures}

1.1 Example images of the dilute and dense phases captured internally by the ITI borescope. . . . . . . . . . . . . . . . . . .

1.2 Example images of the dilute and dense phases captured internally by the Olympus borescope. . . . . . . . . . . . . . . . .

1.3 Example images of the dilute and dense phases captured externally by a telephoto lens. . . . . . . . . . . . . . . .

1.4 Flowchart of the proposed extraction process. $\left\{I_{i}, I_{i+1}, \ldots\right\}$ are images of the flow, $\varepsilon$ is the concentration, and $\mathbf{U}$ is a set of particle velocities. . . . . . . .

1.5 Example of thresholding. The left image is the original and the right is the result of applying a single threshold. . . . . . . . . . . . .

2.1 Applying contrast stretching to an image of the dilute phase captured internally through the ITI borescope. The probability density function shows how the intensities have been spread across the entire range. . . . . . . . .

2.2 Applying contrast stretching to an image of the dilute phase captured internally through the ITI borescope. The pdf shows how the intensities have been spread across the entire range. . . . . . . . . . . . .

2.3 Applying contrast stretching to images of the dilute and dense phase captured internally through the Olympus borescope. . . . . . . . . . . . .

2.4 Applying the histogram equalization transform to an image of the dilute phase captured internally through the ITI borescope. . . . . . . . . . . . .

2.5 Applying the histogram equalization transform to an image of the dilute phase captured internally through the ITI borescope. . . . . . . . . . . . .

2.6 Applying the histogram equalization transform to images of the dilute and dense phase captured internally through the Olympus borescope. . . . . . .

2.7 Applying the local histogram equalization transform to an image of the dilute phase captured internally through the ITI borescope. A window of size $[51 \times$ 51 ] was used. . . . . . . . . . . . . . . . . .

2.8 Applying the local histogram equalization transform to an image of the dilute phase captured internally through the ITI borescope. A window of size $[51 \times$ $51]$ was used. . . . . . . . . . . . . . . . . . . 
2.9 Applying the histogram equalization transform to images of the dilute and dense phase captured internally through the Olympus borescope. A window of size $[101 \times 101]$ was used for each. . . . . . . . . . . . . . . .

2.10 Removing non-uniform illumination from an image taken at the wall of the riser via the top-hat transfrom. . . . . . . . . . . . . .

2.11 Removing non-uniform illumination from an image using the temporal mean. 29

2.12 Example of removing non-uniform illumination using the temporal mean and the top-hat transform. . . . . . . . . . . . . . . . . . .

2.13 Removing high-intensity (salt) and low-intensity (pepper) speckle noise. The median filter was applied with a $[9 \times 9]$ kernel. . . . . . . . . . . . .

2.14 Suppression of high-intensity speckle noise on a temporally adjusted image.

3.1 Set of images $\left\{I_{1078+5 i}\right\}_{i=0}^{8}$ used to determine the baseline data. . . . . . . 38

3.2 Set of images $\left\{I_{1078+5 i}\right\}_{i=9}^{16}$ used to determine the baseline data. . . . . . . 39

3.3 The ground truth (baseline) data manually extracted from the image sequence. 40

3.4 Example of Otsu thresholding on an original image $I(x, y)$ and its adjustment result $I^{\prime}(x, y)=I(x, y)-I_{T}(x, y) \ldots \ldots \ldots \ldots \ldots$

3.5 Localized Otsu thresholding applied to the original images using various block sizes. The image from Figure 3.4(a) has been used with tiles of size $\left\{\left[2^{i} \times 2^{i}\right]\right\}_{i=1}^{9} .44$

3.6 Localized Otsu thresholding applied to the temporally adjusted images using various block sizes. The image from Figure 3.4(c) has been used with tiles of

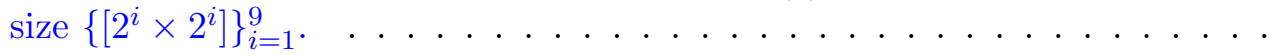

3.7 Particle detection results for variations of the Otsu thresholding method. . .

3.8 Error values for intensity-based methods when compared to the ground truth. The techniques were evaluated on the original images as well as those adjusted to eliminate non-uniform illumination. . . . . . . . . . . . . .

3.9 Error values for intensity-based methods separated into dilute and dense concentration phases. Estimates of the error for the dilute phase, the dense phase, and overall are shown for both image types. . . . . . . . . . .

3.10 Roberts operators . . . . . . . . . . . . . . . . 53

3.11 Prewitt operators . . . . . . . . . . . . . . . . . . 53

3.12 Sobel operators . . . . . . . . . . . . . . . . . . . 54

3.13 Example of gradient approximation and thresholding. The image from Figure 3.4(c) has been used with and without suppressing the specular highlights. 55

3.14 Example of edge thinning. The binary images from Figure 3.13 have been used. . . . . . . . . . . . . . . . . .

3.15 MPE error values for estimating the particle count based on various gradient thresholds. . . . . . . . . . . . . . . . . .

3.16 Results of estimating the particle count by the edge contours. The thresholds used varied from method to method, but each was found to minimize the MPE for that method. . . . . . . . . . . . . . . . .

3.17 Error (MPE) for applying the minimum error thresholds for each method. Both sets of thresholds (minimum MSE and minimum MPE) were tested for each edge detection method on each image type. . . . . . . . . . .

3.18 Error estimates for $k$-NN classifier based on training data. . . . . . . . . . . 
3.19 Example of $k$-NN classification. The left image is the original and the right is the result of the $k$-NN classification. The light, medium, and dark regions

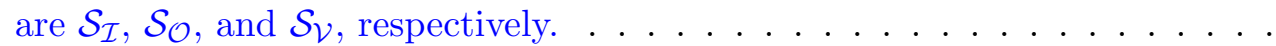

3.20 Results of $k$-NN classification on the set of test images $\mathcal{I}$. Only the training data was collected on the original images, so only they were tested. . . . . .

3.21 Results of estimating the particle count by the number of specular highlights. No account has been made of false positives. . . . . . . . . . . . . .

3.22 Results of estimating the particle count by the number of specular highlights. Edges detected using Canny filter on the $I-I^{s}$ image were dilated by a $[2 \times 2]$ kernel of ones. . . . . . . . . . . . . . . . . . . .

3.23 Results of estimating the particle count by the number of specular highlights. Edges detected using Canny filter on the $I-I^{s}$ image were dilated by a $[3 \times 3]$ kernel of ones. . . . . . . . . . . . . . . . . . . . .

3.24 Error values for estimating the particle count by the number of detected specular highlights. The clipped error values were both for "Adjusted $M_{0}$ " with $E_{\mathrm{MSE}} \approx 143000$ and $E_{\mathrm{MPE}} \approx 3.9 \ldots \ldots \ldots \ldots$

3.25 The ground truth (baseline) data converted to a void fraction in 3 dimensions. 72

4.1 Basic construction of an image pyramid. Level 0 represents the original, high-resolution image; each subsequent step up is a filtered and subsampled version. . . . . . . . . . . . . . . . . . . .

4.2 Example of tracking the boundary of a single particle. The boundary was tracked from from $I_{8}$ to $I_{9}$ using the Lucas-Kanade feature tracker. . . . . .

4.3 An original image and its associated high-intensity noise as determined by the SHS algorithm described in $\S 2.3$. The noise image $I^{s}$ has been transformed using the contrast stretch operator for clarity. . . . . . . . . . . . . . .

4.4 Example of tracking the specular highlight of a particle. The square marks the highlights position in the current image and the circle marks its preceding location. . . . . . . . . . . . . . . . . . .

4.5 Example of tracking the specular highlights. The squares mark the highlight positions in the current image and the circles mark their preceding locations. The X's show unmatched estimated positions; triangles show the previous locations of those unmatched highlights. The images have been cropped to show only about $1 / 8$ of their total area; this has been done for clarity. . . .

4.6 Results of tracking the specular highlights in the sequence $\mathcal{I}$. "Accept" events account for the trajectories in the output of the tracker whereas "Reject" events are those that were detected and eliminated prior to output. . . . . . 
This page is intentionally blank. 


\section{Chapter 1}

\section{Introduction}

\subsection{Motivation}

Fluidized beds are multi-phase ${ }^{1}$ systems widely used in industry for catalytic reactions, solid fuel combustion and gasification, as well as many other applications. In its simplest form, a fluidized bed is a cylinder containing particles which are suspended by a stream of gas injected through the bottom [15]. The presence of these particles and their mobility, specifically between the body of the bed and the combustor wall, increases the heat transfer characteristics of the system [49]. Since the particles are transferring the heat in the system, an increase in velocity will cause in increase in the heat transfer coefficient. For this reason, certain industrial processes require a special variant known as fast beds, or more commonly, circulating fluidized beds [42]. Such processes include catalytic oil cracking, hot-gas cleanup, and coal combustion, carbonization, and gasification [53].

The fluidized bed designs currently in use are borne from years of physically testing and modifying designs to increase their performance [15]. Given the high costs of rigorous experimentation and the relatively low cost of computers, computational fluid dynamics (CFD) is widely being used to help design the next generation of fluid beds. CFD utilizes computers in solving the equations governing fluid-dynamics, such as those of a fluid-bed system, which is an inherently difficult problem [61].

Circulating fluidized beds are characterized by turbulent flows that are physically described by the Navier-Stokes equations. These are the conservation equations of fluid

\footnotetext{
${ }^{1}$ Multi-phase in this context refers to any combination of the three phases or states of matter: gas, liquid, and solid.
} 
mechanics for mass, momentum, and energy of a flow [13]. Even with the fastest computers, these equations are incredibly difficult to solve numerically [18]. For this reason, turbulence models are used that approximate the underlying physics to make the problem computationally tractable.

To determine the accuracy of these CFD models, comparisons must be made to real, empirical data [41]. Unfortunately, this data is difficult to obtain from circulating fluidized beds given their typical high-temperature/high-pressure environments. In order to collect such data, the National Energy Technology Laboratory (NETL), a division of the Department of Energy, has built a small industrial-size cold-flow circulating fluidized bed (CFB) [47]. This CFB is a low-temperature/low-pressure unit, but the particle material can be chosen such that its properties while fluidized compare well to other materials commonly used in industrial applications [41].

The low-temperature/low-pressure environment of the CFB makes it possible for researchers to access the flow stream via off-the-shelf probes. One probe of particular interest that is currently being used by the Computational Sciences Division - the NETL group responsible for this CFB - is a borescope coupled to a high-speed digital camera. The borescope is a small tube with a lens system built inside that can be inserted into the flow stream. The attached high-speed camera is then able to capture high-resolution images of the internal structure of the flow.

Another benefit of using the cold-flow CFB was the ability to replace sections of the system with acrylic glass. This gives researchers visual access to the particle flow and the motion of its structure from the outside. Taking advantage of these transparent sections, the high-speed camera has also been used with a telephoto lens to capture particle behavior at the cylinder wall.

From the experimental images, either captured through the borescope or the telephoto lens, it is expected that high-quality data can be extracted through advanced image processing techniques. The individual velocities of particles in the flow and the local concentrations of particles would be of great use to NETL modelers. The extraction of such data by automated processes motivates this project. 


\subsection{Problem}

Techniques for visualizing the flow of fluids have long been sought to aid in designing experiments and to help interpret measurements from local point probes. Early applications often gave only qualitative observations of the flow behavior, but digital image processing techniques have allowed for the extraction of highly precise, quantitative data from images of the flow [22]. Many of the original implementations of digital processing techniques to these images were in an attempt to describe the behavior of large-scale structures in the flow [22]. The motion of large structures found in a CFB, namely particle clusters and strands, are often analyzed due to their significant role in the heat transfer within the riser [9]. However, if particles can be imaged separately then information on their geometry can be extracted, such as size, shape, and position relative to the large-scale structures [22].

Flow visualization techniques can be separated into two broad categories: internal and external visualization. To visualize the flow internally, some type of optical probe must be inserted into the flow stream. This impedes the flow, possibly changing its behavior, but allows for capturing images across the entire radius of the containment vessel, e.g., the riser. External techniques have the benefit of being non-intrusive and therefore do not affect the flow in any way, but these have limited applications and are often unable to describe radially dependent behavior.

\subsubsection{Internal Visualization}

Optical fiber probes have long been used to determine particle velocities and concentrations in fluid systems, as well as the size and velocity of particle clusters [36, 63, 50]. As point probes, however, they are unable to provide any information on the shape of the clusters [26]. To obtain this information and a deeper understanding of particle-particle interactions within the flow of a CFB, internal imaging techniques have been developed using observation tubes [40, 57], fiber scopes [21], and optical scopes, i.e., borescopes/endoscopes, [35, $56,32]$ with various methods for illuminating the particle flow.

Takeuchi et al. [57] used an observation tube - a simple tube of an undisclosed material - with a thin glass plate covering the end inserted in the riser. A high-speed video camera was positioned in front of the tube to capture the particle behavior which was illuminated by a xenon light introduced through a light guide (a bundle of optical 
fibers). The light guide was opposite the observation tube, illuminating the particles from behind. The experiment was an attempt to study the shape, frequency, and velocity of particle streamers. Automated analysis by particle image velocimetry (PIV $)^{2}$ software was attempted but failed due to the low quality of the images. Their analysis was based on a manual, visual assessment of the experimental images.

Hatano et al. [21] used two types of fiber scopes: a reflective-type and a penetrativetype. The reflective-type used a large, central optical fiber to transmit the visible particle behavior to a camera. The particle surfaces were illuminated by light from a halogen lamp, transmitted into the riser by a number of small fibers arranged around the central fiber. The penetrative-type used two large optical fibers whose ends faced each other. One fiber was used to transmit the visible particle behavior to a video camera and the other was used to transmit light from a halogen lamp into the flow, illuminating the particles from behind. From a manual analysis, they reported the particle count and velocity range across a short sequence of frames. In analyzing the experimental images they observed a "blackout" during the passing of a dense swarm of particles and divided the area of this dark region by the mean particle area to determine a particle count.

Li et al. [35] used an optical probe with a lens-barrel wrapped in optical fibers. The barrel housed a set of lenses for transmitting the images to an attached video camera and the fibers were used to transmit light pulses from a xenon lamp into the flow. Through their technique, a number of micrographs were captured at each of a few radial positions in the riser and then used to identify two phases of the flow: a dispersed phase and a cluster phase. The dispersed phase was said to contain predominantly separated particles and the cluster phase contained particle groups or agglomerations. They then determined, presumably visually, the number of micrographs with clusters and divided this by the total number of micrographs to give an estimate of the cluster frequency.

Takeuchi and Hirama [56] used a bore scope (lens-barrel) and a high-speed video camera to capture the flow behavior. A light guide was inserted opposite the bore scope and was used to transmit halogen light into the flow, illuminating particles from behind. Like Hatano et al. [21], they described periods of blackout in the frame sequence during the passing of dense clusters. Their analysis was mostly qualitative, aimed at describing the

\footnotetext{
${ }^{2}$ Particle image velocimetry (PIV) typically refers to a dense-phase application of pulse-light velocimetry (PLV) where an auto- or cross-correlation technique is used to determine the average displacement of an interrogation window $[2,15]$. Images captured for PLV are usually illuminated by a powerful, pulsed laser light sheet.
} 
behavior of clusters during different states of the $\mathrm{CFB}$, such as fast fluidization and dilute transport.

Lackermeier et al. [32] applied a laser light sheet to the problem of internal flow visualization. An endoscope was used to transmit laser light into the flow with a cylindrical lens at the end for creating a laser sheet. Another endoscope, inserted perpendicular to the light sheet, was used to observe the reflectance of the light off the moving particles. The benefit and their reason for using the light sheet was the well-defined sampling volume of the illuminated region. However, the laser power required for decent illumination was a major drawback. Unable to adequately employ PIV or particle tracking velocimetry (PTV $)^{3}$ techniques to determine single particle velocities, but capable of identifying a lean and a dense phase, they resigned to investigate the behavior of clusters and strands. Basic image processing techniques were used to filter out individual particles (seen as highly intense speckle noise), identify clusters through thresholding, and combine the detected clusters from multiple frames to "visualize" them as a whole.

The images of the internal flow originally captured by the team at NETL were taken by a high-speed digital camera and a borescope with a wide-angle field of view. The borescope, manufactured by Instrument Technology, Inc. (ITI), was essentially a lens-barrel with a light guide wrapped around it. When inserted in the flow, a halogen lamp connected to the light guide illuminated the particle surfaces, which was then transmitted back to the camera. The setup was very similar to that of Li et al. [35] and Hatano et al. [21] (the reflective-type).

The images captured by this system were typically very dark, making it nearly impossible to distinguish the particles from the background. This was especially true under highly dilute conditions where, given the relatively small accumulated surface area of the particles, only a small portion of the light projected into the flow was transmitted back to the camera. Dense conditions, however, produced brighter images since the collective surface area of the particles was large, reflecting a large amount of light into the camera. Although the separation between gas and solids phases became more pronounced, little could be done to differentiate between individual particles.

Example images of those captured through the ITI scope for the lean (disperse)

\footnotetext{
${ }^{3}$ Particle tracking velocimetry (PTV) usually refers to a dilute-phase application of pulse-light velocimetry (PLV) where individual particles are tracked either across multiple frames or using the streaks created by overexposure of a single frame for multiple light pulses $[2,15]$. Like PIV, this technique often employs a high-powered, pulsed laser sheet to illuminate the particles.
} 
phase and the dense (cluster) phase are given in Figure 1.1. As they are, quantifying the location of individual particles or their velocities would be very difficult. Individual particles can be distinguished in the lean phase, but it is still impossible to determine their locations given the absence of any depth information. It may be possible to simply threshold the images and, like Hatano et al. [21], estimate the particle count by dividing the area of the particle or cluster regions by the mean particle area. Otherwise, it is obvious that a series of pre-processing steps are required before any other information can be extracted from these images.

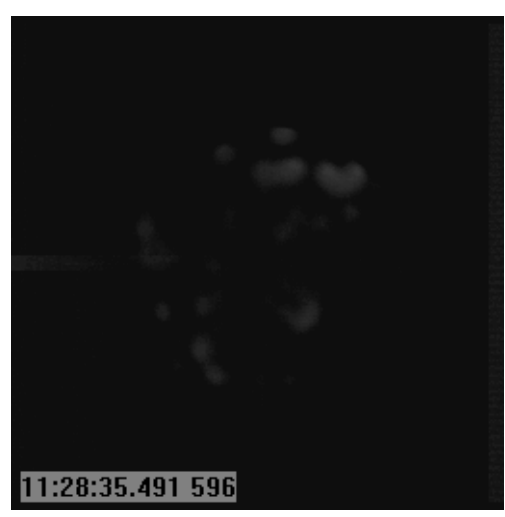

(a) Dilute phase

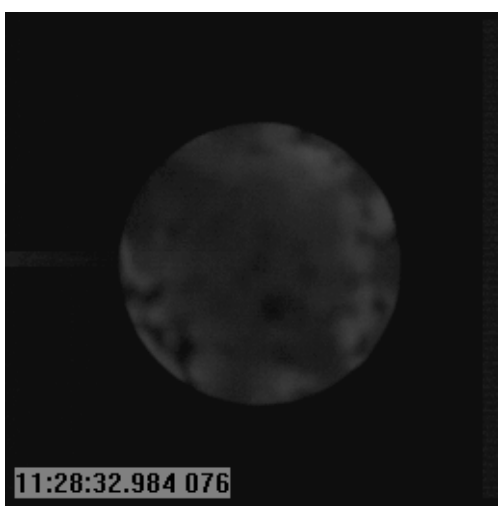

(b) Dense phase

Figure 1.1: Example images of the dilute and dense phases captured internally by the ITI borescope.

More recently, a different borescope has been used, this one manufactured by Olympus, with a narrower field of view. Unlike the ITI borescope where essentially everything visible was in-focus, the Olympus model has a focal-point about $8.5 \mathrm{~mm}$ from the tip of the probe. Objects on this focal plane, perpendicular to the line-of-sight, will be in-focus and have known coordinates in three-dimensional space. The well-defined focal plane makes the Olympus borescope a more attractive option than the ITI scope, where the position of an object can only truly be estimated in relation to other visible objects; its coordinates in three-dimensional space are impossible to determine independently.

Other improvements include a much brighter halogen light source and the addition of a series of magnification lenses between the camera and the borescope. Through the extra lenses, the image can be magnified to fill the entire CCD array in the camera, maximizing the amount of information extracted from the flow. Examples of images captured through 
the Olympus scope for the lean phase and the dense phase are given in Figure 1.2.

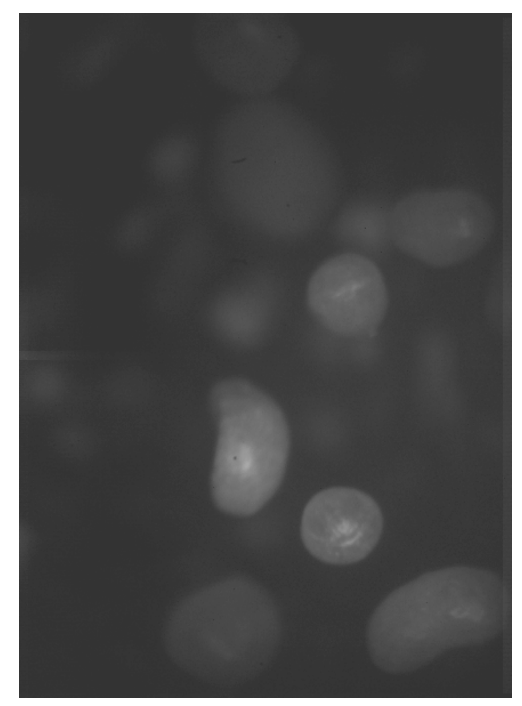

(a) Dilute phase

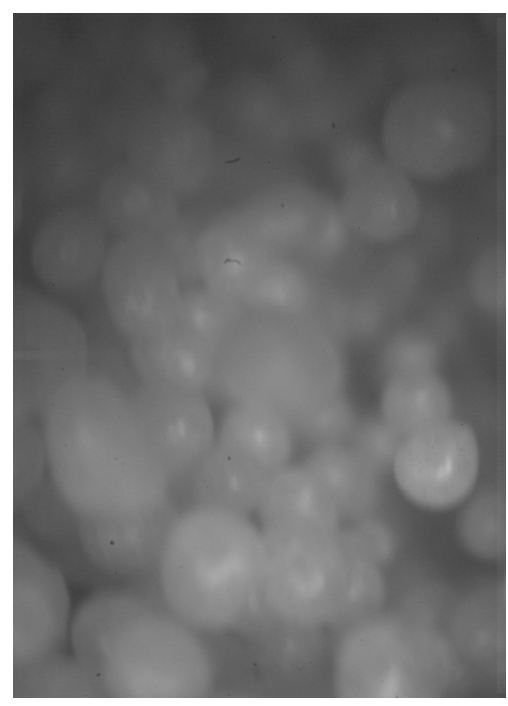

(b) Dense phase

Figure 1.2: Example images of the dilute and dense phases captured internally by the Olympus borescope.

\subsubsection{External Visualization}

Probe techniques, like those previously mentioned, physically impede the flow, changing its characteristics and adding error to the measurements being made [14]. An alternative is to visualize the internal flow from outside the system. Several non-invasive techniques exist, including capacitance imaging and $\gamma$-ray tomography, but these are typically only capable of obtaining a single measurement, such as the solids volume concentration [62]. Imaging techniques, on the other hand, allow for multiple measurements simultaneously; however, serious limitations apply. One major limitation is the requirement for visual access to the flow; another often being a limit on the solids concentration at the wall [27]. These often restrict such techniques from industry applications but the measurements being made are vital to developing complete models for fluidized beds [62, 36]. Many of the experiments conducted using non-invasive imaging techniques have been made on gas-solid fluid systems [48, 27, 9, 51, 54], but gas-liquid [52] and even three-phase, gas-liquid-solid systems [14] have been studied.

Rhodes et al. [48] externally captured the gas-solid flow of a CFB riser through 
a glass section by a high-speed video camera and a lamp used for illumination. Their experiments were directed toward the study of particle motion at the riser wall. In their study, three forms of particle motion were identified: dilute flow, dense flow, and swarm flow. These flows were defined as follows: dilute - very dilute with uniform motion; dense somewhat dense with uniform motion; and swarm - large group of particles packed together tightly. The video was analyzed manually but they were able to report the distribution of mean downward velocities and residence times for particle swarms during various operating conditions.

Horio and Kuroki [27] used a set of laser sheets and a video camera to externally visualize the internal flow across a vertical and horizontal plane in a bubbling fluidized bed. They concluded that if the mass flow rate of the solids, $G_{s}$, is larger than $0.6 \mathrm{~kg} / \mathrm{m}^{2} \mathrm{~s}$ then the density of the flow along the riser wall will prohibit external visualization of the internal flow structure. Given this, their experiments were limited to lower solids flow rates. The mean size and mean rise velocities of gas pockets and clusters were reported based on a manual analysis.

Bredebusch et al. [9] investigated the flow structure at the riser wall of a CFB through a transparent silica section. A high-speed camera with a telephoto lens was used to capture images, but they limited the image width to between 10 and $15 \mathrm{~cm}$ in consideration of the curvature of the transparent section. A photo lamp was set at an angle of $45^{\circ}$ relative to the camera's line of sight. The placement was meant to increase the visible structure in the image by inducing shadows. Preprocessing methods were applied to the images to reduce measurement errors. They estimated and reduced the systematic error by finding the mean value for each pixel in a sequence of images and then divided each of those images by the previously determined temporal mean image. Contrast stretching was later applied to increase visibility. Unable to discern individual particles, an attempt was made to determine the velocity field of the visible particle structure using optical flow ${ }^{4}$. The distribution of velocities for two operating conditions was reported.

Saadevandi and Turton [51] observed the gas-solid flow of a spouted fluid bed through a section of flat, transparent glass. Illuminated by a back lighting technique, the particle behavior was captured with a video camera; the video was later digitized. Two measurements were desired for their study: radial voidage profiles and radial velocity

\footnotetext{
${ }^{4}$ The optical flow technique implemented by Bredebusch et al. [9] was a multi-scale version of the classic Horn and Schunck optical flow [28] as described by Bergen et al. [5].
} 
profiles. They were able to interrogate various depths within the flow by calibrating the focus using a manually constructed bed of particles. To calculate the voidage (void fraction), a fast shutter speed $(0.1 \mathrm{~ms})$ was used to ensure the images contained crisp particles, i.e., no streaking, and then applied a series of automated tests to determine if particles were present as well as in focus. The reported void fractions were based on the count of in-focus particles. For calculating the particle velocities, a slow shutter speed (1 to $2 \mathrm{~ms}$ ) was used to induce particle streaking. The length of the streaks was directly proportional to the velocity of the particles. A gradient-based method was used to identify streaks of in-focus particles and then a searching scheme was applied to find their endpoints and, thus, the length. This technique was unable to discriminate between positive (up) and negative (down) velocities.

Shi [54] investigated the behavior of clusters in a CFB using a laser sheet and high-speed digital camera. Four classes of particle clusters were defined, differentiated by size and particle separation. The classifications ranged from a few particles moving together to a highly dense strand of particles often found at the wall. An intensity threshold was applied to distinguish the solids from the background and then, based on their classification scheme, focused their analysis on clusters only. They reported a radial profile of the mean axial velocities in the positive (up) and negative (down) directions, having determined the instantaneous cluster velocities using PIV.

Seol et al. [52] applied basic image processing techniques and a combination of PIV and PTV to images captured of a two-phase, liquid-gas bubble plume. A laser sheet was used to illuminate the flow which was captured by a set of three high-speed digital cameras arranged as a column. To help characterize the behavior of the liquid (water), the flow was seeded with non-fluorescent tracer particles. Having observed a significant difference in the reflected intensities of the bubbles and tracer particles, an intensity threshold was applied to separate the two phases, creating an image of the bubble phase alone. The flow field of the bubble phase was then determined by PTV. The flow field of the original two-phase image is determined by PIV and, using the flow vectors determined for the bubble phase alone, filtered to determine the velocity field of the liquid phase. The distribution of velocities for each phase showed good agreement with expected values.

Chen and Fan [14] applied a laser light sheet and a high-speed digital camera to a 3-phase (gas-liquid-solid) fluidized bed. High- and low-pass filters were applied to the experimental images to remove noise before being binarized by a threshold on the intensity ${ }^{5}$.

\footnotetext{
${ }^{5}$ Pixels in a binary image take on only two values, typically 1 or 0 . Here, the values are referred to as
} 
A binary image is one with just two values, typically 0 and $1 \mathrm{~A}$ search routine was employed that, upon encountering a marked pixel in the binary image, traced the boundary of an object to determine its centroid and mean radius. Based on their associated radius, objects were classified as one of three types: solid particles (solid-phase), seeded particles (assume properties of the liquid-phase), and bubbles (gas-phase). After the classification process, displacement vectors were determined by linking intra-class objects across three successive frames. The linking was accomplished by increasing the size of a circular search window until 3 centroids $\left(\mathbf{c}_{\mathbf{1}}, \mathbf{c}_{\mathbf{2}}, \mathbf{c}_{\mathbf{3}}\right)$, each from a separate frame $\left(f_{1}, f_{2}, f_{3}\right)$, were found with similar displacements $\left(\Delta\left(\mathbf{c}_{1}, \mathbf{c}_{2}\right) \approx \Delta\left(\mathbf{c}_{\mathbf{2}}, \mathbf{c}_{\boldsymbol{3}}\right)\right)^{6}$. Using the spatial displacement between centroids and the temporal displacement between frames, they obtained instantaneous velocities for the different phases of the system.

The NETL team has applied external visualization techniques to the particle flow along the wall of the riser. The same high-speed camera mentioned previously was used with a telephoto lens to focus just beyond the inner surface of a tranparent, acrylic riser section. The image size is kept small, typically about $16 \times 16 \mathrm{~mm}^{2}$, to mitigate the effect of geometric distortion due to the curvature of the riser. Example dilute and dense phase images from this system are shown in Figure 1.3.

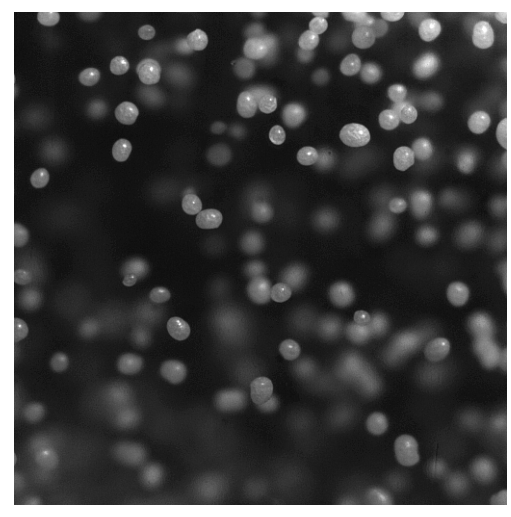

(a) Dilute phase

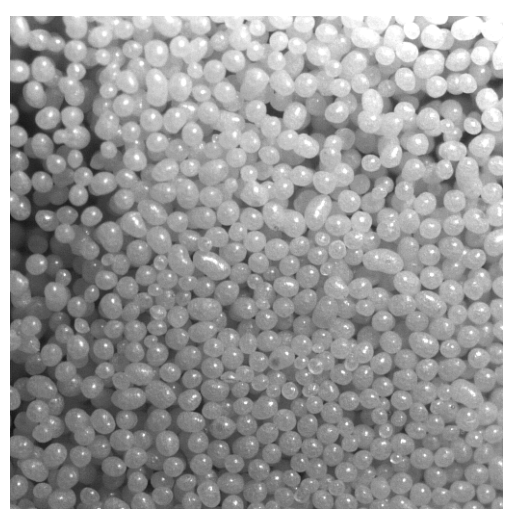

(b) Dense phase

Figure 1.3: Example images of the dilute and dense phases captured externally by a telephoto lens.

marked or unmarked where the marked pixels are usually of significant interest.

${ }^{6} \Delta(\mathbf{a}, \mathbf{b})$ is used here as shorthand for the Euclidean distance between two vectors $\mathbf{a}$ and $\mathbf{b}$, namely $\Delta(\mathbf{a}, \mathbf{b})=\|\mathbf{a}-\mathbf{b}\|_{2}=\left(\sum_{i}\left(a_{i}-b_{i}\right)^{2}\right)^{1 / 2}$ 


\subsection{Project Goals}

The aim of this project is to effectively process digital images supplied by NETL to extract particle information, specifically local particle concentrations and individual particle velocities. The particle concentration can presumably be determined from any single frame in the sequence by summing the areas of all particles found in the image, specifically those that are in-focus, along the focal-plane. Extracting the velocity of a single particle requires detecting that same particle in at least two frames, or images, in the sequence. This spatial displacement normalized by the temporal difference between frames gives the velocity.

Each goal can be achieved independently of the other. Knowing the velocity of one particle across a few frames does not affect the concentration estimate of any single image. Likewise, the total concentration of one image does not affect determining the velocity estimate of a single particle. Electing to achieve each of these goals independently, the major goals of this project become:

1. Given an arbitrary frame $I_{k}$, identify the regions of in-focus particles.

2. Given an arbitrary frame $I_{k}$, identify individual in-focus particles.

3. Given an arbitrary frame $I_{k}$ from a video sequence, its successor $I_{k+1}$, and the location of in-focus particles in both, track the particles from one to the other.

\subsection{Proposed Solution}

\subsubsection{The Approach}

The proposed approach to achieving the major goals discussed earlier is a set of single-output functions that work individually on specific parts of the problem. As long as each is effective at their task, it should be possible to thread these functions together to produce both types of information - particle concentrations and velocities - seemingly at once.

This approach, shown in Figure 1.4, requires three major functions (large, solidline boxes) and two minor functions (small, dotted-line boxes). The major functions do most of the work for achieving the project goals, but the minor functions are required for the conversion of intermediate data produced by the major functions into the specific units required by NETL, i.e, velocities in meters or feet per second and the concentration 
based on either particles, $\varepsilon_{p}$, or voidage, $\varepsilon_{v}$. The particle concentration and voidage are complementary measurements where $\varepsilon_{p}+\varepsilon_{v}=1$.

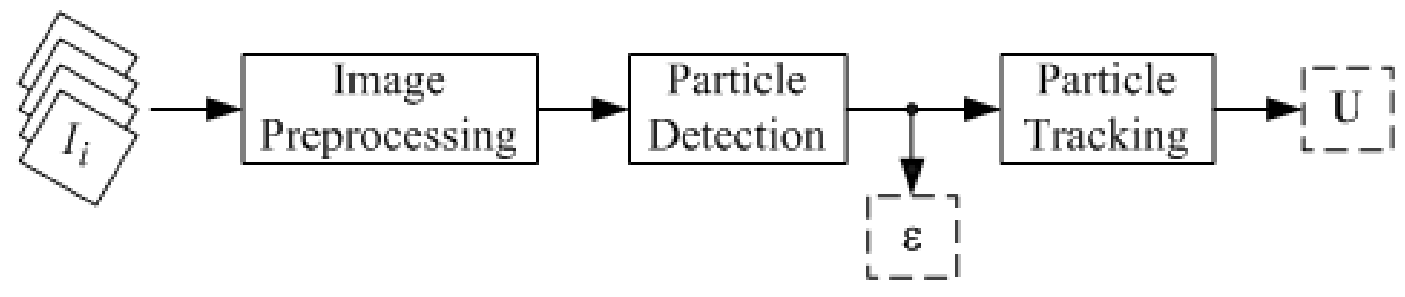

Figure 1.4: Flowchart of the proposed extraction process. $\left\{I_{i}, I_{i+1}, \ldots\right\}$ are images of the flow, $\varepsilon$ is the concentration, and $\mathbf{U}$ is a set of particle velocities.

\subsubsection{Functional Requirements}

The three major functions are Image Preprocessing, Particle Detection, and Particle Tracking. Each requires some basic input and, once complete, returns a single output. The two minor functions are labeled by their output data types: $\varepsilon$ is the concentration and $\mathbf{U}$ represents a set of velocities.

\section{Image Preprocessing}

Depending on the quality of the input images and the types of features that must be extracted, preprocessing techniques may need to be applied. These can range from reducing or eliminating noise and illumination non-uniformities to enhancing contrast or edges. These techniques will be discussed in Chapter 2 .

\section{Particle Detection}

Given a set of images, determine the locations of all visible particles, especially those on the focal-plane. Since the location of the focal-plane is known, if these particles can be identified then their position in three-dimensional space will be known. Techniques employed to manage this task are discussed in Chapter 3.

\section{Particle Tracking}

Given the locations of particles on the focal-plane in a sequence of images, track the particles from one frame to the next. This will give the spatial displacement of each particle 
and, since the temporal displacement between frames is known, will allow for computing the instantaneous velocity. Potential tracking schemes are discussed in Chapter 4.

\section{Minor Functions}

The minor functions are conversion functions. One is needed to take a count of particles in an image and determine the local particle concentration $\varepsilon$. The other is needed to take a set of trajectories or velocities in pixels per frame and convert them to meters or feet per second. These minor functions are described within Chapters 3 and 4 as needed.

\subsubsection{Advantages}

The main advantage to this approach is the ability to quickly and easily replace a routine that either inadequately solves its intended problem or fails altogether. This helps to quickly produce a working prototype and makes algorithmic improvements much easier to implement.

One example would be to initially use a single threshold to separate the particles from the background, thereby "detecting" them. This will produce a binary image and, depending on the input image, could correctly mark the pixels corresponding to particles. In reality, the threshold will misclassify pixels and produce discontinuous regions across individual particles as well as eliminate boundaries between neighbors. These effects can be seen in Figure 1.5.

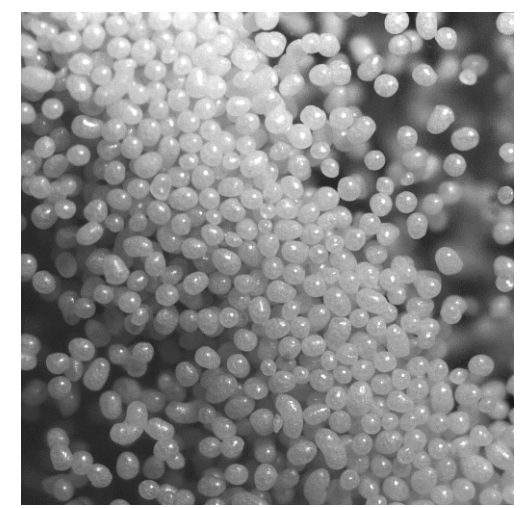

(a) Original Image

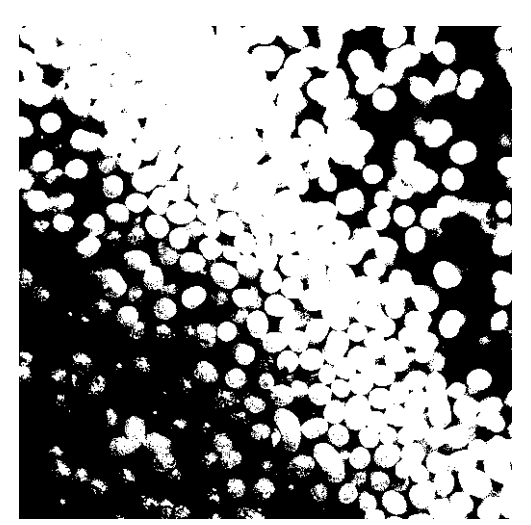

(b) Threshold Result

Figure 1.5: Example of thresholding. The left image is the original and the right is the result of applying a single threshold. 
At the outset, applying this technique along with an assumption of negligible or zero noise would allow for the research effort to initially concentrate on tracking alone while still having a complete working model. Once the tracking method has been well defined, the segmentation and boundary extraction techniques could easily be replaced by more sophisticated algorithms. 


\section{Chapter 2}

\section{Image Preprocessing}

Preprocessing techniques are often necessary prior to information extraction in order to enhance image features [8] and can depend on the equipment used for capturing the images [22]. Many enhancement techniques are available to increase contrast, sharpen edges, reduce noise, etc., but the criteria for choosing the appropriate preprocessing steps is difficult to quantify and becomes more of a trial and error exercise [22, 8]. A few examples of enhancement techniques are presented here to address problems inherent in the flow images captured by NETL researchers.

\subsection{Contrast Enhancement}

When capturing images of the flow internally, a very powerful light source must be used to adequately illuminate the flow [22]. The amount of light reflected to the camera is also dependent on the particle density in the image plane [15]. Therefore, inadequate lighting and dilute particle concentrations can cause the images to be very dark with a limited contrast between particles and the empty background. An example of this effect can be seen in Figure 1.1(a) where the particles are very difficult to locate visually.

To counter this effect, a number of contrast enhancement techniques can be applied to expand the histogram of the image across the dynamic range. Histogram modification is a basic image processing technique that does not increase the information content and, in general, will not decrease it either [7, 12]. However, the enhancement can assist in visually analyzing the images [12] while increasing the effectiveness of classification techniques [8]. 


\subsubsection{Contrast Stretching}

Contrast stretching is the simplest enhancement technique available to stretch the pixel intensities across the dynamic range of grayscale values. The pixel intensities of the images captured by the high-speed camera are represented as unsigned 8-bit integers providing $L=2^{8}$ different intensity levels over the range of possible discrete values $\mathcal{R}$. $\mathcal{R}$ is typically expressed in one of two forms, either $\mathcal{R}=\mathcal{R}_{\mathbb{Z}^{+}}=[0, L-1]$ where each value is a positive integer or $\mathcal{R}=\mathcal{R}_{\mathbb{R}}=\frac{\mathcal{R}_{\mathbb{Z}^{+}}}{\max \left\{\mathcal{R}_{\mathbb{Z}^{+}}\right\}}=[0,1]$ where each value is a discrete floating point. Oftentimes computations are more straightforward when using the latter form, thus for simplicity, $\mathcal{R}=\mathcal{R}_{\mathbb{R}}$ should be assumed unless explicitly stated otherwise.

Given an image $I$ with $n$ rows and $m$ columns, for all pixels $(x, y) \in I$, such that $x \in \mathcal{X}=\{1,2, \ldots, n\}$ and $y \in \mathcal{Y}=\{1,2, \ldots, m\}$, a transformation operator $T[\cdot]$ can be applied to $I$ to form a modified image $I^{\prime}$ by $[20,7]$

$$
I^{\prime}(x, y)=T[I(x, y)] \forall x \in \mathcal{X}, y \in \mathcal{Y} .
$$

The contrast stretch operation is a linear transformation of the form

$$
T[I(x, y)]=a \cdot I(x, y)+b
$$

where $a$ is a positive scaling term that can expand $(a>1)$ or contract $(a<1)$ the histogram and $b$ is an offset that can brighten $(b>0)$ or darken $(b<0)$ the overall image [7].

To modify the histogram, $a$ and $b$ must be determined such that the maximum and minimum values in $I$ are mapped to the maximum and minimum values in $I^{\prime}$. These new extrema in $I^{\prime}$ can be explicitly set to that of the entire intensity range $R$, but $a$ and $b$ can be generalized to allow for any new extremum provided they remain within $R$. Solving the transformation equation in (2.2) for the two extrema gives the following:

$$
\begin{aligned}
a \cdot \min (I)+b & =\min \left(I^{\prime}\right), \\
a \cdot \max (I)+b & =\max \left(I^{\prime}\right)
\end{aligned}
$$


where the functions $\min (\cdot)$ and $\max (\cdot)$ are defined as

$$
\begin{aligned}
\max (I) & =\max _{x \in \mathcal{X}, y \in \mathcal{Y}}\{I(x, y)\} \\
\min (I) & =\min _{x \in \mathcal{X}, y \in \mathcal{Y}}\{I(x, y)\} .
\end{aligned}
$$

Subtracting (2.3) from (2.4) and rearranging gives

$$
a=\left(\frac{\max \left(I^{\prime}\right)-\min \left(I^{\prime}\right)}{\max (I)-\min (I)}\right)
$$

Substituting (2.7) into (2.3) and rearranging gives

$$
b=\min \left(I^{\prime}\right)-\min (I)\left(\frac{\max \left(I^{\prime}\right)-\min \left(I^{\prime}\right)}{\max (I)-\min (I)}\right) .
$$

Finally, plugging both (2.7) and (2.8) into (2.2) and rearranging gives the general contrast stretching transformation of $I$ to $I^{\prime}$ as

$$
\begin{aligned}
I^{\prime}(x, y) & =T[I(x, y)] \\
& =(I(x, y)-\min (I))\left(\frac{\max \left(I^{\prime}\right)-\min \left(I^{\prime}\right)}{\max (I)-\min (I)}\right)+\min \left(I^{\prime}\right) .
\end{aligned}
$$

As mentioned earlier, the extrema of $I^{\prime}$ can be set as those of $R$ by letting $\min \left(I^{\prime}\right)=\min \{R\}$ and $\max \left(I^{\prime}\right)=\max \{R\}$ in (2.10) which will expand the image histogram to cover the entire available grayscale range.

Examples under dilute and dense-case conditions are shown in Figures 2.1 and 2.2 , respectively. The result of each transformation appears substantially improved over the original image. The images were cropped prior to applying the transform in order to remove the timestamp from the bottom (seen in Figure 1.1). Figure 2.3 shows examples of the dilute and dense phase captured using the Olympus borescope. The Olympus images already used most of the grayscale range, which limited the effectiveness of this method.

As opposed to showing the histogram, the more general probability density function (pdf) of the image intensities is given in Figures 2.1 and 2.2. The probability of each intensity level given an input image $I$ with intensity levels in the space $\mathcal{L}=\{1,2, \ldots, L\}$ can be defined as

$$
p\left(l_{k}\right)=\frac{N_{k}}{N}, \text { for } k \in \mathcal{L},
$$


where $l_{k}$ is the $k^{t h}$ intensity level, $N_{k}$ is the number of pixels in $I$ with intensity $l_{k}$, and $N=n \cdot m$, the total number of pixels in image $I$. This constructs the pdf of the intensity levels in $I$ which has the useful properties that $0 \leq p\left(l_{k}\right) \leq 1$ and $\sum_{k} p\left(l_{k}\right)=1[34]$.

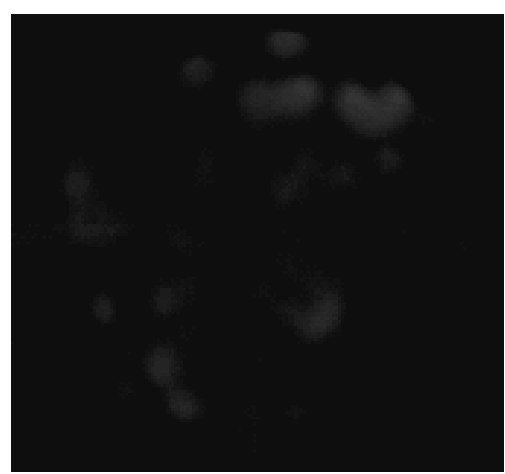

(a) Original image $I(x, y)$

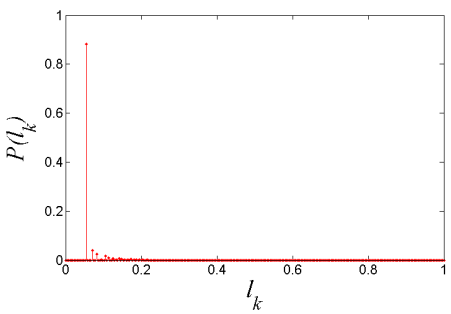

(c) Original pdf

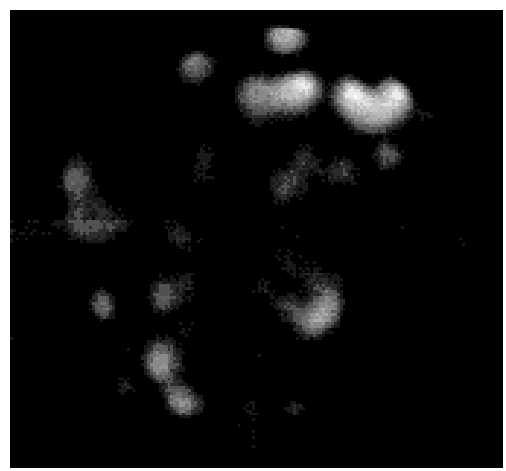

(b) Contrast stretched image $I^{\prime}(x, y)$

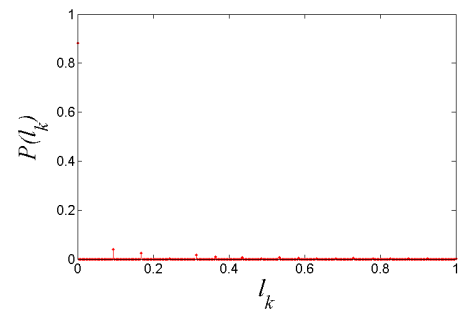

(d) pdf after contrast stretching

Figure 2.1: Applying contrast stretching to an image of the dilute phase captured internally through the ITI borescope. The probability density function shows how the intensities have been spread across the entire range.

One problem commonly associated with the contrast-stretching transformation is its inability to deal appropriately with outlying intensities. If the intensity extrema in image $I$ coincide with that of $R$, the transformation will not alter the original image. One method that can be employed to address this problem is the use of a bandpass filter to attenuate a small portion of intensities at the ends of the distribution [20]. Technically this will decrease the information content of the output but the loss could be small and necessary for enhancement. This was not an issue for images captured with the ITI borescope after being cropped. However, if the timestamps had been left in the images, their pure black digits and white background would have kept the transformation from effectively altering the images. 


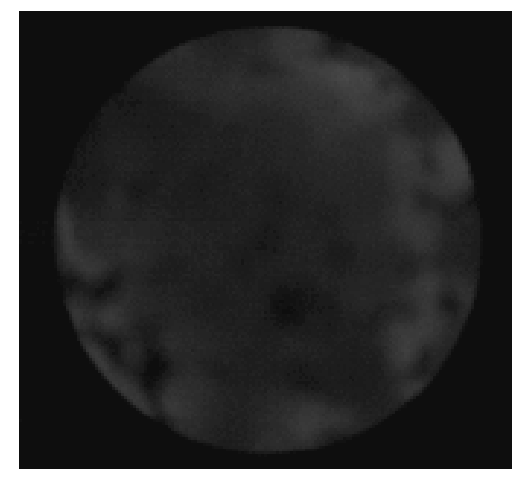

(a) Original image $I(x, y)$

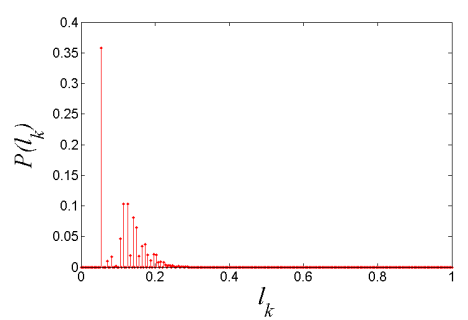

(c) Original pdf

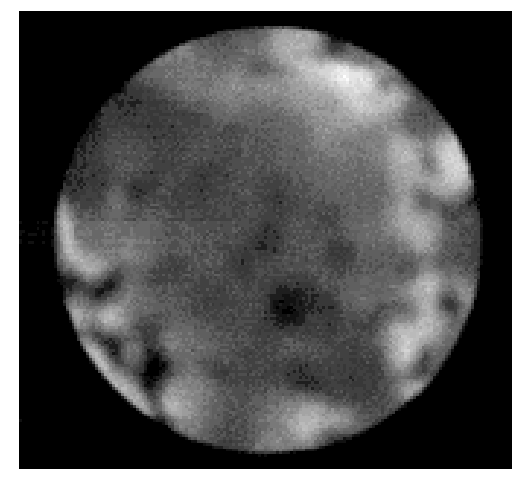

(b) Contrast stretched image $I^{\prime}(x, y)$

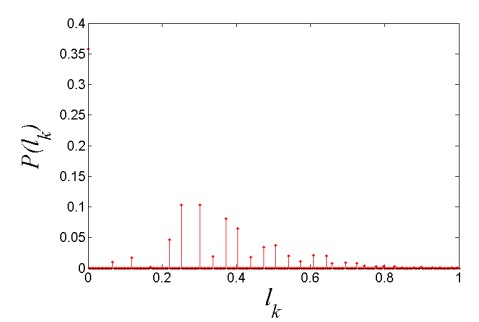

(d) pdf after contrast stretching

Figure 2.2: Applying contrast stretching to an image of the dilute phase captured internally through the ITI borescope. The pdf shows how the intensities have been spread across the entire range.

\subsubsection{Histogram Equalization}

Another method used to enhance the contrast of an image is histogram equalization. In histogram equalization (HE), information gleaned from the intensity histogram of the image guides the transformation process [20]. HE attempts to transform the image such that the histogram of the processed image is uniform, making each gray level equiprobable $[12,58]$.

The cumulative distribution function (cdf) of a discrete system $F(\cdot)$ is the cumulative sum of the probabilities [23] thus for an image $I$ with gray-levels $l_{k}$ for $k \in \mathcal{L}$, the cdf is defined as

$$
\begin{aligned}
F\left(l_{k}\right) & =\operatorname{Pr}\left[I \leq l_{k}\right] \\
& =\sum_{i=1}^{k} p\left(l_{i}\right) .
\end{aligned}
$$




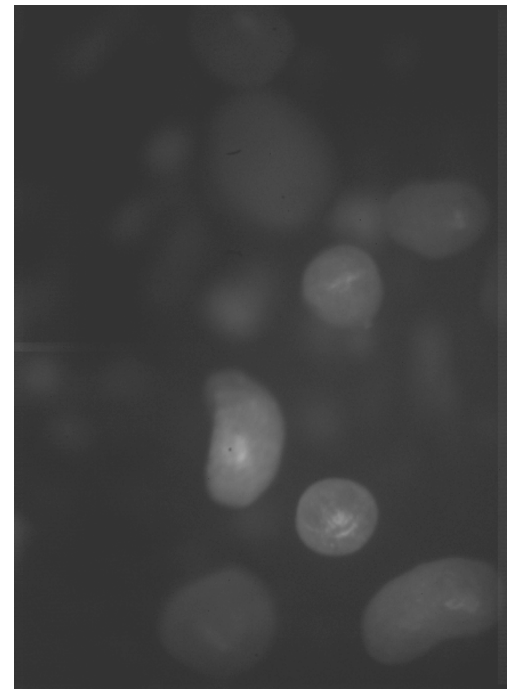

(a) Original dilute image $I(x, y)$

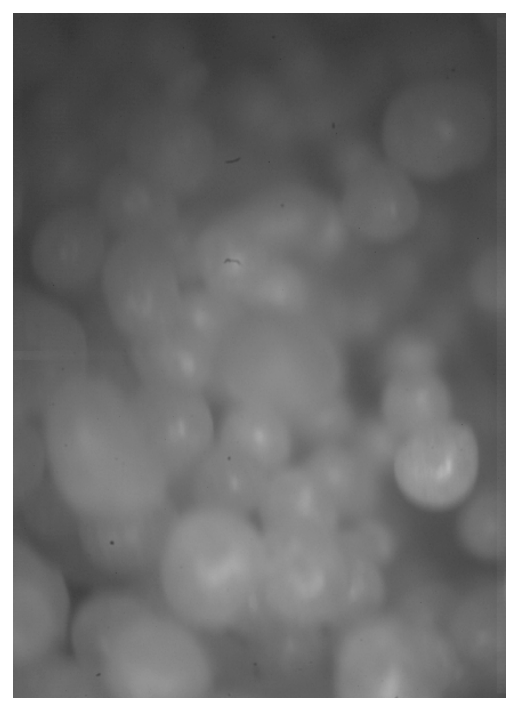

(c) Original dense image $I(x, y)$

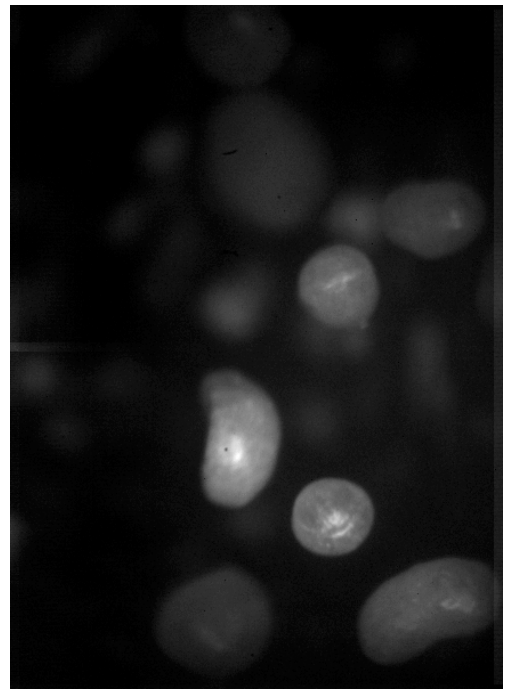

(b) Contrast stretched dilute image $I^{\prime}(x, y)$

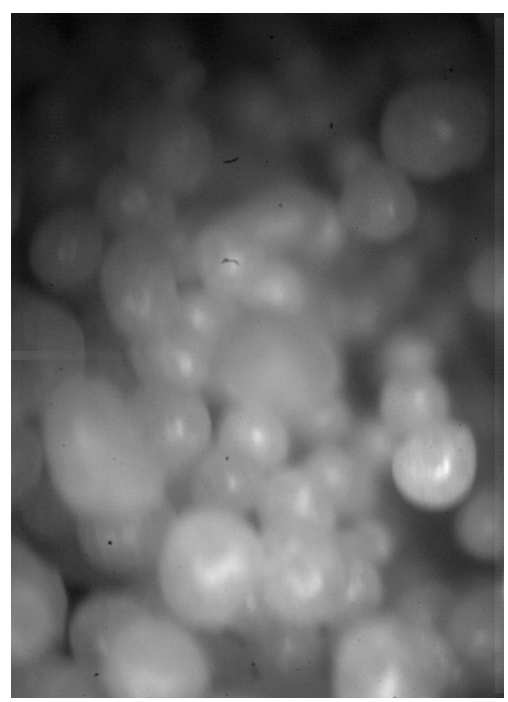

(d) Contrast stretched dense image $I^{\prime}(x, y)$

Figure 2.3: Applying contrast stretching to images of the dilute and dense phase captured internally through the Olympus borescope. 
The HE transformation function actually is the cdf of the intensity levels and can be described more concisely as a gray-level transformation as opposed to a pixelwise transformation, e.g., contrast stretching $[7,20]$. Thus, the gray-level $l_{k}^{\prime}$ in $I^{\prime}$ is

$$
\begin{aligned}
l_{k}^{\prime} & =F\left(l_{k}\right) \\
& =\sum_{i=1}^{k} p\left(l_{i}\right) .
\end{aligned}
$$

Once the transformation of all gray-levels has been completed, it can be useful to apply the contrast stretch transform on the result [7]. Another slight modification is to use

$$
l_{k}^{\prime}=\frac{1}{2}\left(F\left(l_{k}^{\prime}\right)-F\left(l_{k-1}^{\prime}\right)\right)+F\left(l_{k-1}^{\prime}\right)
$$

which essentially places the gray-level at the center of the histogram bin as opposed to the right edge, as in $l_{k}^{\prime}=F\left(l_{k}\right)[16]$. Figures 2.4 and 2.5 show the result of applying the histogram equalization transform to the dense and dilute ITI borescope images and Figure 2.6 show it for those of the Olympus borescope. The form of the transformation defined in (2.16) was used without applying the contrast stretch transform.

In Figure 2.4 it is not difficult to see the consequences of using (2.16) instead of (2.14). In the histogram of the transformed image the majority of pixels are near the middle of the grayscale, having moved from an intensity of almost 0 in the original. If the general definition of histogram equalization had been used, these pixels would have been pushed close to an intensity of 1 , saturating the transformed image even more. The effect on the dense-case example in Figure 2.5 would be much less noticeable.

While the transformation has indeed increased the dynamic range of the image, the results are still inadequate. There is a distinction between the particles and the background but much of the detail needed to discern individual particles is lost in the transformation.

\subsubsection{Local Histogram Equalization}

Local Histogram Equalization (LHE) is useful for enhancing small image details that can be neglected by the generic, global HE method described in the previous section [16]. The basic idea is to define a window, or neighborhood, such that when centered around a pixel in $I$, the neighborhood of pixels will be used to define the local histogram 


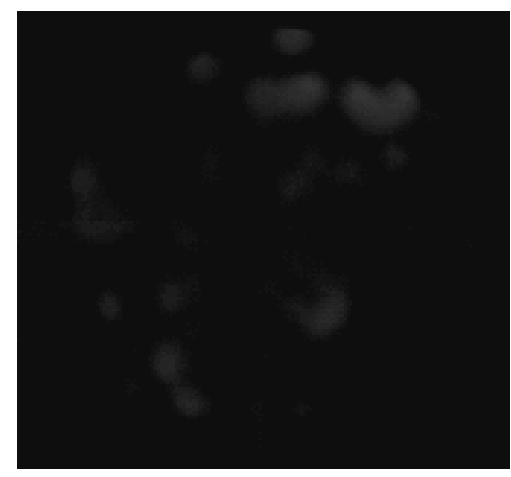

(a) Original image $I(x, y)$

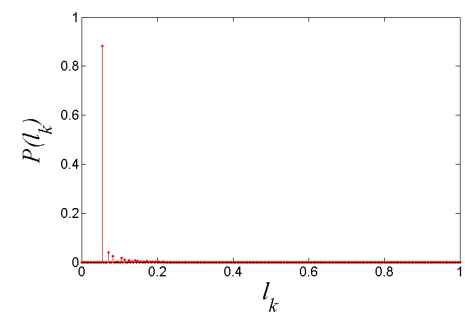

(c) Original pdf

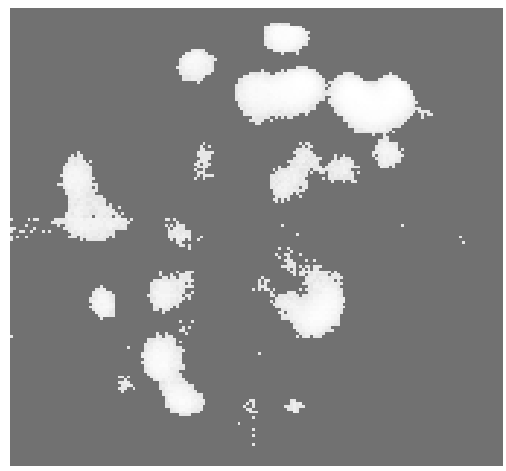

(b) HE image $I^{\prime}(x, y)$

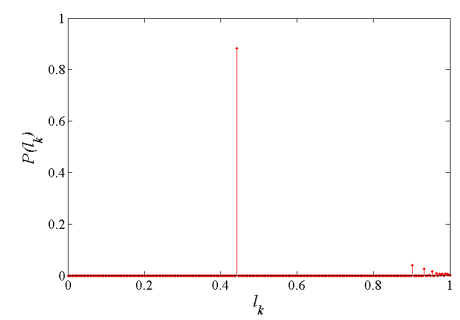

(d) pdf after HE

Figure 2.4: Applying the histogram equalization transform to an image of the dilute phase captured internally through the ITI borescope.

which will in turn be used to determine the transformation intensity for the central pixel [16].

Results of applying LHE to the ITI images using a [51 $\times 51]$ window are shown in Figures 2.7 and 2.8 for the dilute and dense phases, respectively. The Olympus images were transformed using a window of size $[101 \times 101]$ and the results are shown in Figure 2.9. The transformation results in images that are less saturated than those of traditional HE, but a significant amount of noise has been added or at least magnified. The magnified noise is especially noticeable in Figures 2.8(b) and 2.9(b) and can depend a great deal on the window size [16].

\subsection{Eliminating Non-uniform Background Illumination}

The team at NETL chose a constant halogen light source to directly illuminate the image plane. Ideally, this illumination would be perpendicular to the plane but this is difficult given the presence of the camera. Light can be directed toward the flow by a light guide constructed around a borescope, but external visualization of the CFB requires 


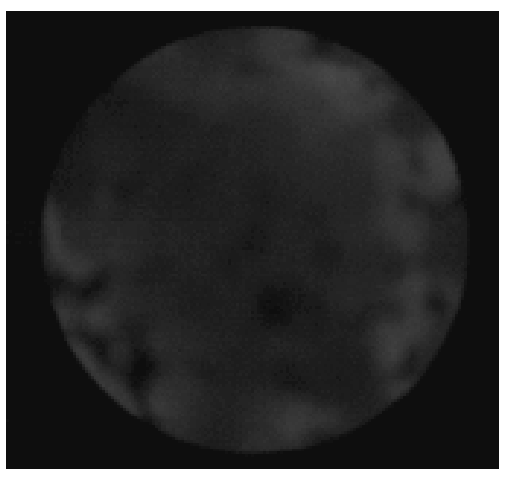

(a) Original image $I(x, y)$

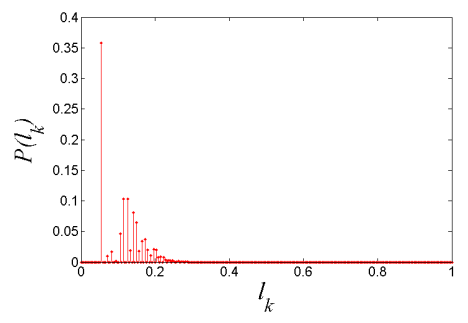

(c) Original pdf

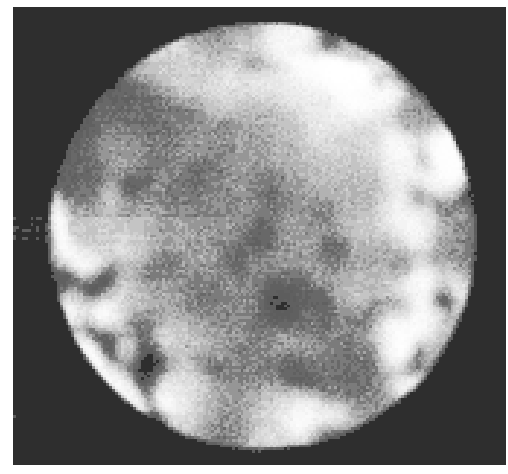

(b) HE image $I^{\prime}(x, y)$

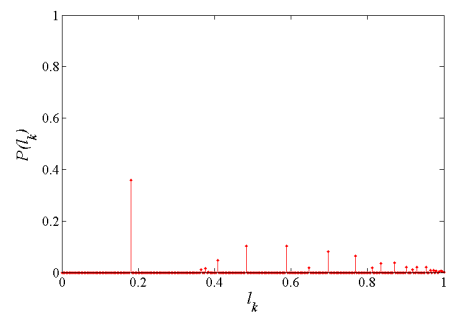

(d) pdf after HE

Figure 2.5: Applying the histogram equalization transform to an image of the dilute phase captured internally through the ITI borescope.

a larger light source which must be applied at an angle to the image plane. Applied in this way, the light source can non-uniformly illuminate the image and, subsequently, those particles visible in it. This effect can be a subtle discrepancy in the intensity of the surface of a particle as it travels across the surface of the plane or can be much more extreme where areas of an image are fully saturated with light. An example of this extreme case is shown in Figure 2.10(a) and is the specific problem at hand. To eliminate this undesired lighting effect, two general avenues exist: (1) estimate the lighting for a single image, or (2) estimate the lighting across time from a sequence of images.

\subsubsection{Top-hat Transformation}

To estimate the lighting for a single image, a top-hat transformation can be applied [20]. The transformation first estimates the background lighting and subtracts it for each image individually. The estimate of the background lighting is based on the morphological opening of the original image using a disk-shaped structuring element (SE). The 


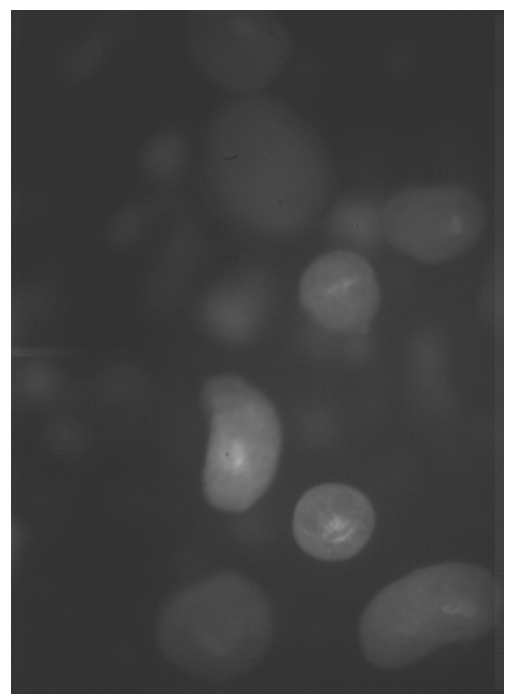

(a) Original dilute image $I(x, y)$

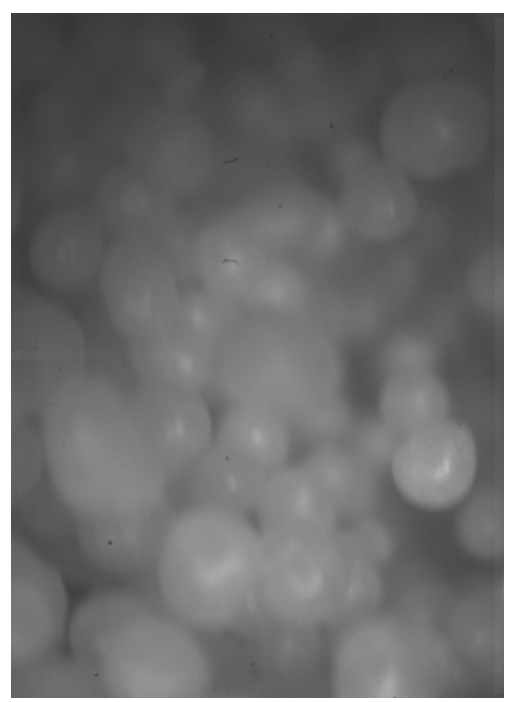

(c) Original dense image $I(x, y)$

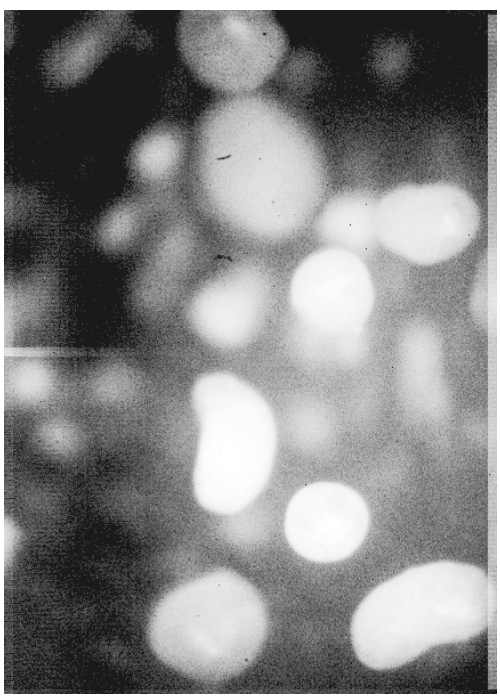

(b) HE dilute image $I^{\prime}(x, y)$

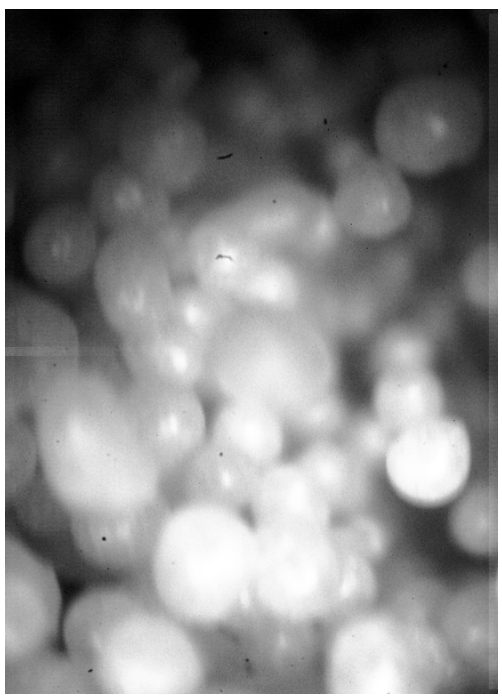

(d) HE dense image $I^{\prime}(x, y)$

Figure 2.6: Applying the histogram equalization transform to images of the dilute and dense phase captured internally through the Olympus borescope. 


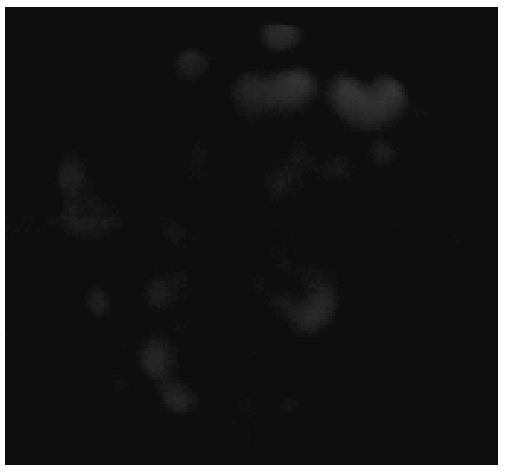

(a) Original image $I(x, y)$

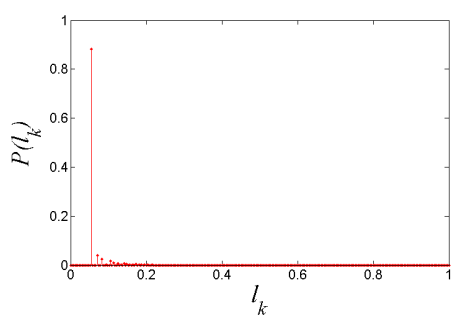

(c) Original pdf

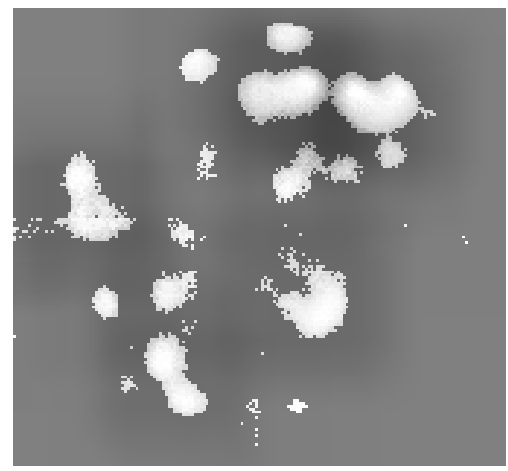

(b) LHE image $I^{\prime}(x, y)$

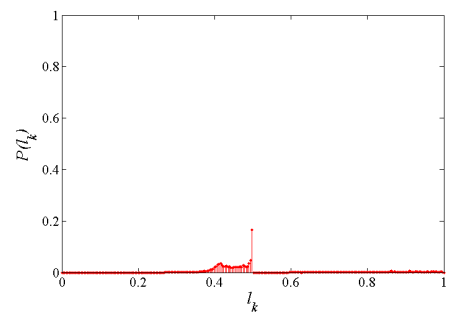

(d) pdf after LHE

Figure 2.7: Applying the local histogram equalization transform to an image of the dilute phase captured internally through the ITI borescope. A window of size $[51 \times 51]$ was used.

opening operator is defined as the combination of first eroding the image using the SE and then dilating the result using the same SE. Opening the image effectively smoothes the intensity gradients in the image by blurring small objects into the background resulting in a good estimate of the variation in the lighting. Once the top-hat transformation has been performed, the histogram of the transformed image can be readjusted to increase its contrast.

A dense-flow example image is shown in Figure 2.10(a) where the illumination nonuniformity is apparent, specifically by the saturation at the top of the image. Images of the estimated lighting, the top-hat transformation, and the readjusted result for the example are all shown in Figure 2.10. Particles in the final image, Figure 2.10(d), are darker but the illumination is more uniform which is the ultimate goal of this transformation. 


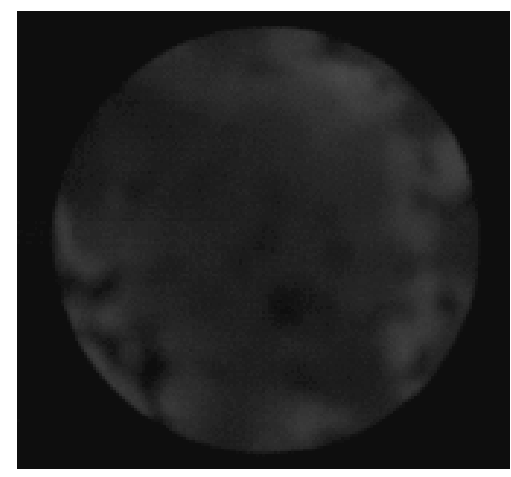

(a) Original image $I(x, y)$

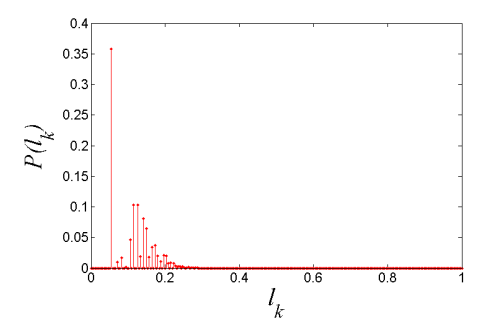

(c) Original pdf

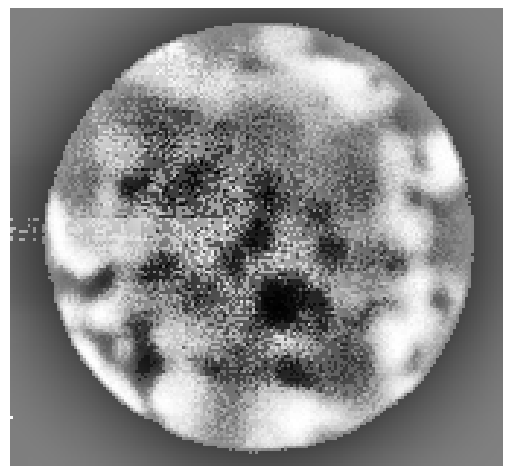

(b) LHE image $I^{\prime}(x, y)$

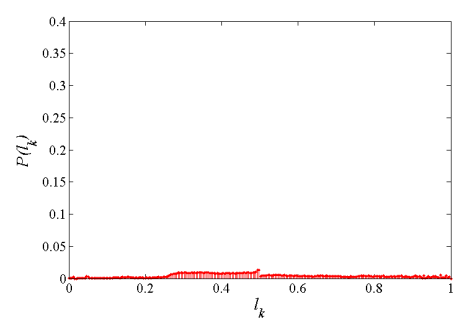

(d) pdf after LHE

Figure 2.8: Applying the local histogram equalization transform to an image of the dilute phase captured internally through the ITI borescope. A window of size $[51 \times 51]$ was used.

\subsubsection{Temporal Mean}

To estimate the lighting for a sequence of images whose lighting, while non-uniform, remains constant, the temporal mean of the lighting can be determined [9]. The temporal mean is simply the mean intensity of each pixel across all frames of the sequence. Given a sequence of images $\mathcal{S}$, the temporal mean image $I_{T}$ can be represented as:

$$
I_{T}(x, y)=\frac{1}{|\mathcal{S}|} \sum_{s \in \mathcal{S}} I_{s}(x, y) \forall x \in \mathcal{X}, y \in \mathcal{Y}
$$

Similar to the top-hat transform, the estimate $I_{T}$ is subtracted from every image in the sequence $\mathcal{S}$ and the resultant images can be readjusted to increase their contrast.

An example of using the temporal mean is shown in Figure 2.11 where the image from Figure 2.10(c) has again been used. As would be expected, the temporal mean gives a smoother approximation of the lighting and, thus, a smoother transformation result. Figure 2.12 shows a comparison of the two transformations where their differences are more 


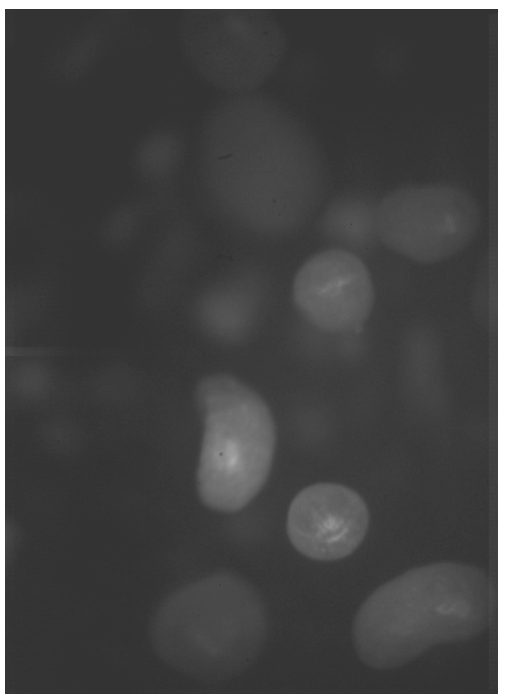

(a) Original dilute image $I(x, y)$

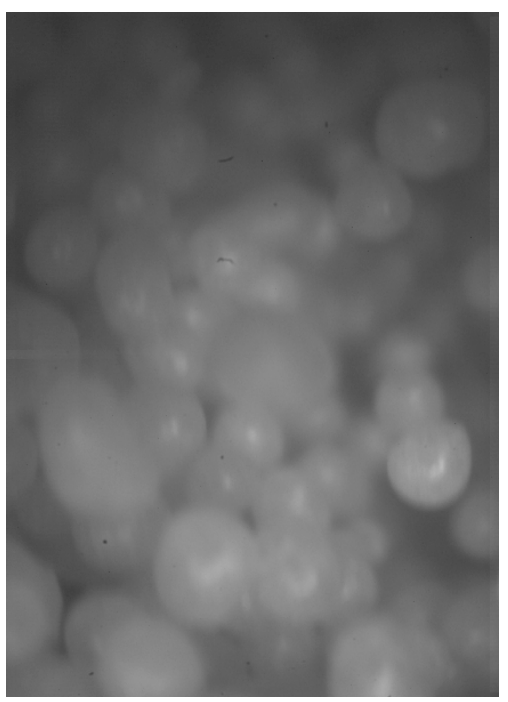

(c) Original dense image $I(x, y)$

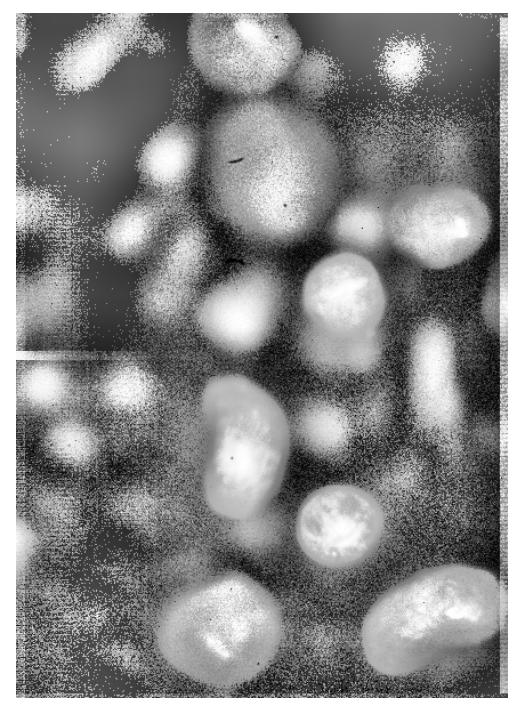

(b) LHE dilute image $I^{\prime}(x, y)$

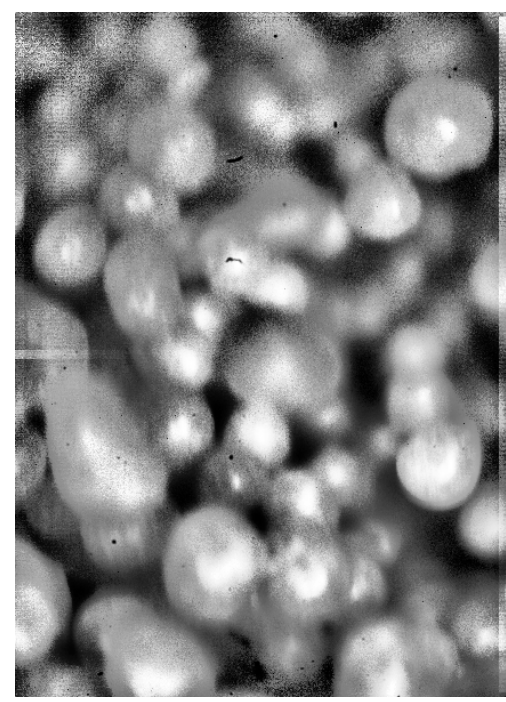

(d) LHE dense image $I^{\prime}(x, y)$

Figure 2.9: Applying the histogram equalization transform to images of the dilute and dense phase captured internally through the Olympus borescope. A window of size $[101 \times 101]$ was used for each. 


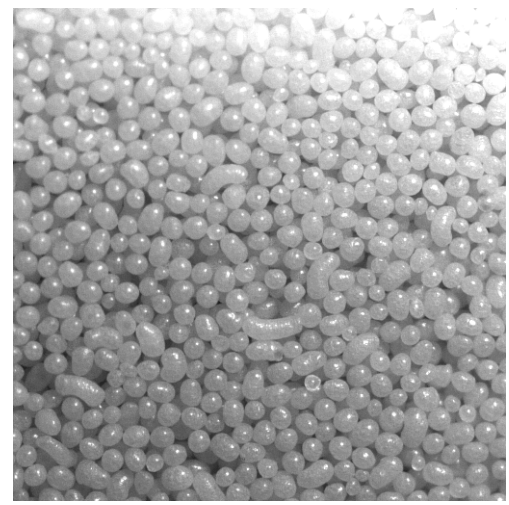

(a) Original image $I(x, y)$

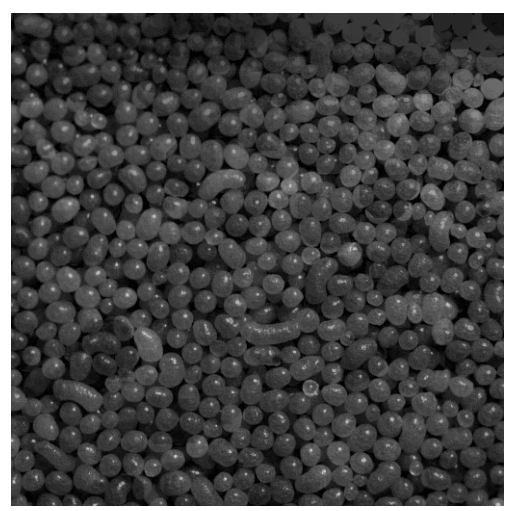

(c) Top-hat result $J=I-I^{O}$

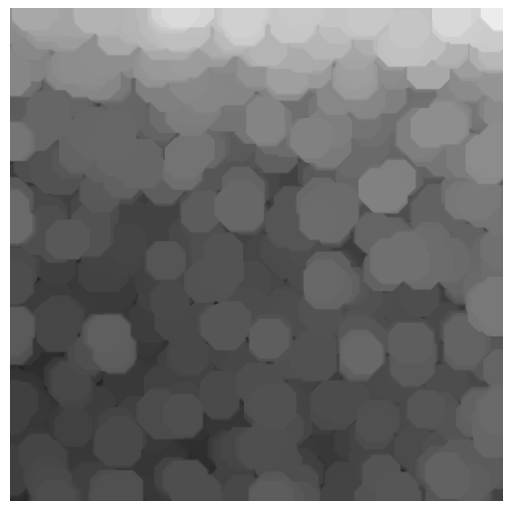

(b) Lighting estimate $I^{O}(x, y)$

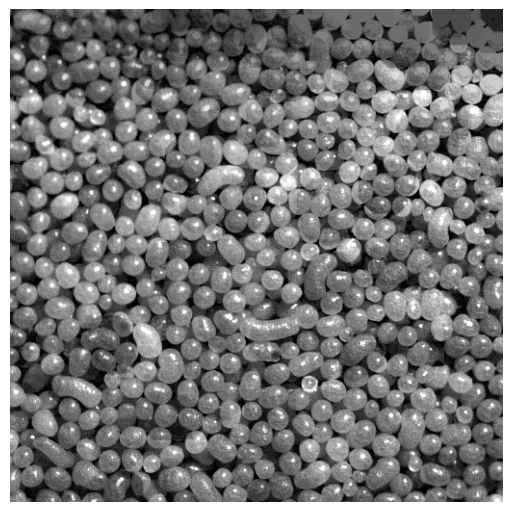

(d) Adjusted result $J^{\prime}(x, y)$

Figure 2.10: Removing non-uniform illumination from an image taken at the wall of the riser via the top-hat transfrom.

pronounced.

One major difference is due to the the top-hat transform being dependent only on the current image. Since there are few particles in the top-right corner where the saturation is most prevalent - clearly visible in Figure 2.11(a) — it is not accounted for in the lighting estimation shown in Figure 2.12(b). The other significant difference is the presence of artifacts from the structuring element which are visible as intensity discontinuities across individual particles in the top-hat transformation result.

\subsection{Specular Highlight Suppression}

Specular highlights are caused by a mirror-like reflection of light off smooth, glossy surfaces $[10,43]$. These reflections are often treated as noise but can be useful for object 


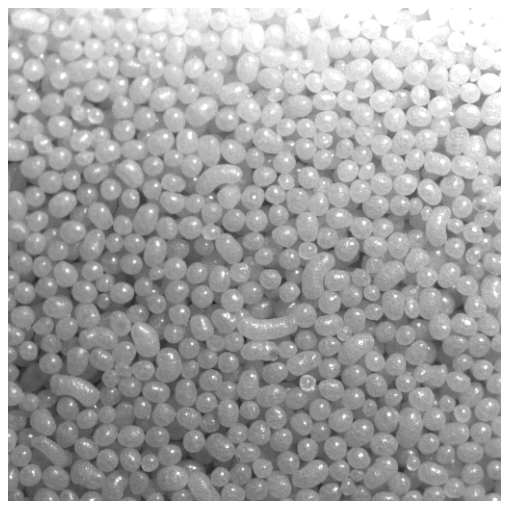

(a) Original image $I(x, y)$

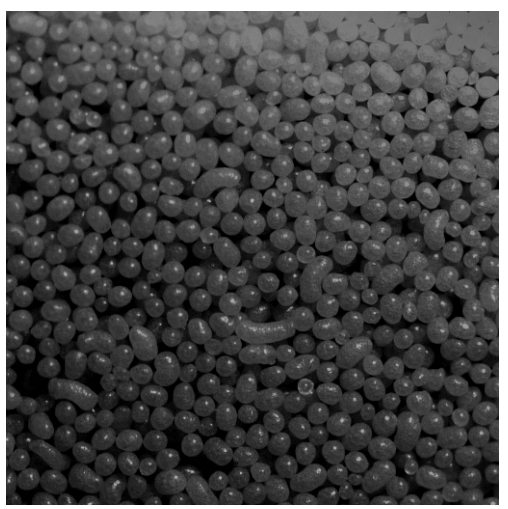

(c) Difference $K=I-I_{T}$

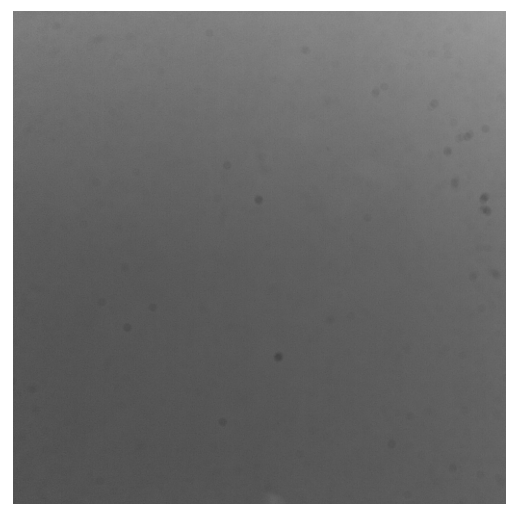

(b) Temporal mean $I_{T}(x, y)$ across $\mathcal{S}$

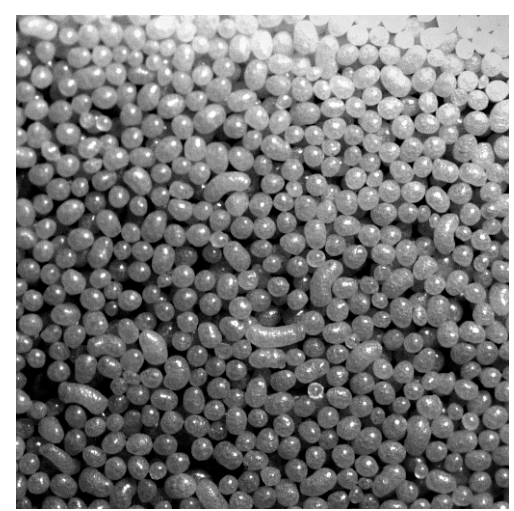

(d) Adjusted result $K^{\prime}(x, y)$

Figure 2.11: Removing non-uniform illumination from an image using the temporal mean.

recognition [44], determining camera motion [43], and even determining surface shape [55] but cannot be used for estimating depth of the surface [10]. In the experimental images, the presence of specular highlights is generally restricted to in-focus particles, but are occasionally found on out-of-focus particles close to the image plane. The highlights present a problem for edge detection methods since they create strong gradients across the surfaces of particles. In this case it would be preferable to suppress these bright spots while minimizing the loss of any other salient details from the image.

Intensely bright highlights caused by collimated light (as opposed to natural, ambient light) can be identified by simply applying an intensity threshold to the image [10, 44]. Because of its simplicity, however, this can drastically overestimate the number of specularities which must then be individually accepted or rejected based on other criteria [44]. 


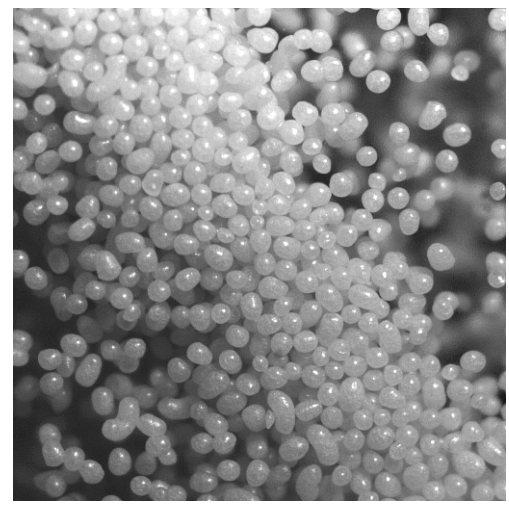

(a) Original Image

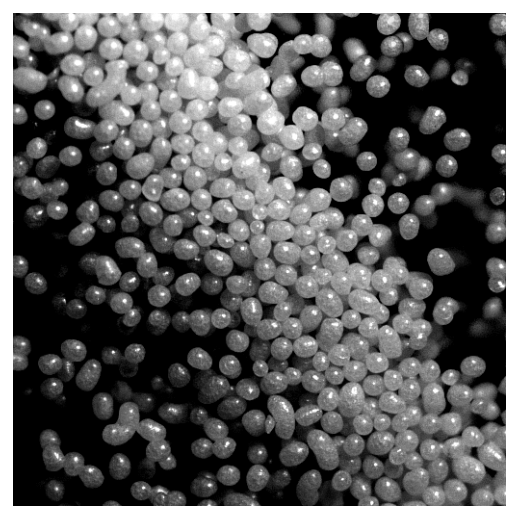

(c) Adjusted Temporal Mean Image

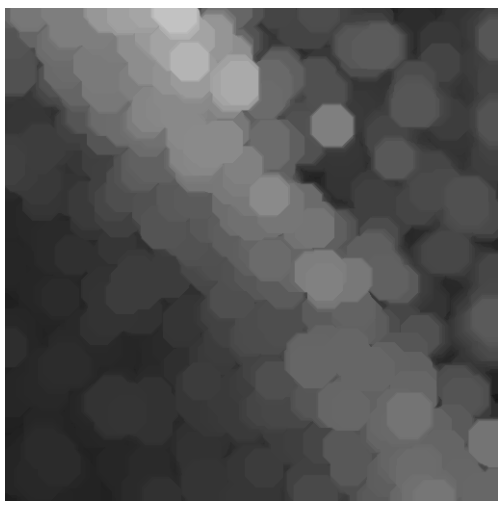

(b) Top-hat Lighting Estimate

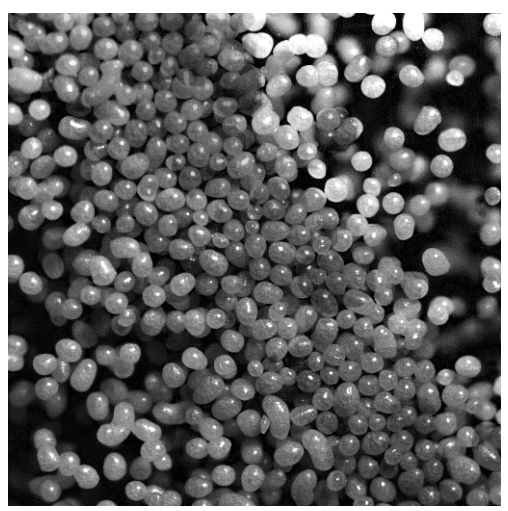

(d) Adjusted Top-hat Result

Figure 2.12: Example of removing non-uniform illumination using the temporal mean and the top-hat transform.

The accepted (detected) highlights would then need to be suppressed or smoothed into the original image. Instead, a more robust method is defined that will directly suppress the specularities.

Median filters, though simple, are commonly used to remove salt-and-pepper noise [20]. A window of user-specified dimensions is passed over the image and, as the window passes across a neighborhood of pixels, the center pixel is given the median intensity of that neighborhood. As a statistic, the median serves as a robust estimate of the center of a distribution [23]. Applied in this fashion, any outlying intensities in these small neighborhoods, both high and low, are suppressed.

For the application at hand, it is only necessary or even desirable to suppress the high-intensity outliers in the image corresponding to specular highlights. To do so, the filter 
or its output must be necessarily separated to distinguish between the two types of noise: high-intensity outliers, or "salt", and low-intensity outliers, or "pepper". It turns out the key is the filter output and the separation is fairly trivial.

To effectively separate the two, only one observation must be made: high-intensity noise (salt), when suppressed, will result in lower pixel intensities in the output image than in the original and vice-verse for low-intensity noise (pepper). Given an image $I(x, y)$ and the output of applying the median filter to it, $I^{M}(x, y)$, define $I^{D}(x, y)$ as the difference of the filtered result from the original:

$$
I^{D}(x, y)=I(x, y)-I^{M}(x, y) .
$$

Based on the earlier observation, all of the pixels affected by "salt" noise should be represented by positive values in $I^{D}$, while those effected by "pepper" should be negative. Now define two new matrices $I^{s}$ and $I^{p}$ as

$$
\begin{aligned}
& I^{s}(x, y)=\left\{\begin{array}{ccc}
I^{D}(x, y) & \text { if } & I^{D}(x, y)>0 \\
0 & \text { if } & I^{D}(x, y)<0
\end{array}\right. \\
& I^{p}(x, y)=\left\{\begin{array}{ccc}
I^{D}(x, y) & \text { if } & I^{D}(x, y)<0 \\
0 & \text { if } & I^{D}(x, y)>0
\end{array}\right.
\end{aligned}
$$

such that $I^{D}=I^{s}+I^{p}$. Desiring only to remove the "salt", $I^{s}$ is subtracted from the original image $I$ giving the final result $I^{\prime}$. Working backward, it is easily shown that this process suppresses the high-intensity noise while preserving the remaining image.

$$
\begin{aligned}
I^{\prime}(x, y) & =I(x, y)-I^{s}(x, y) \\
& =I(x, y)-\left\{\begin{array}{ccc}
I^{D}(x, y) & \text { if } & I^{D}(x, y)>0 \\
0 & \text { if } & I^{D}(x, y)<0
\end{array}\right. \\
& =I(x, y)-\left\{\begin{array}{ccc}
I(x, y)-I^{M}(x, y) & \text { if } & I(x, y)-I^{M}(x, y)>0 \\
0 & \text { if } & I(x, y)-I^{M}(x, y)<0
\end{array}\right. \\
& =I(x, y)-\left\{\begin{array}{ccc}
I(x, y)-I^{M}(x, y) & \text { if } & I(x, y)>I^{M}(x, y) \\
0 & \text { if } & I(x, y)<I^{M}(x, y)
\end{array}\right.
\end{aligned}
$$

Evaluating the two conditions of (2.24) gives: 
Case $1\left(I(x, y)>I^{M}(x, y)\right)$

$$
\begin{aligned}
I^{\prime}(x, y) & =I(x, y)-\left(I(x, y)-I^{M}(x, y)\right) \\
& =I(x, y)-I(x, y)+I^{M}(x, y) \\
& =I^{M}(x, y)
\end{aligned}
$$

Case $2\left(I(x, y)<I^{M}(x, y)\right)$

$$
\begin{aligned}
I^{\prime}(x, y) & =I(x, y)-0 \\
& =I(x, y)
\end{aligned}
$$

Thus only pixels affected by high-intensity noise are altered. Alternatively, if only the lowintensity noise is to be removed, one simply needs to subtract $I^{p}$ from the original image instead. Figure 2.13 shows the result of using each half of the filter separately, as well as the traditional, combined version on an original image. The result of suppressing high-intensity noise in a temporally adjusted image is shown in Figure 2.14.

\subsection{Summary}

The flow images captured externally by a telephoto lens will be the subject of the following study. The absence of occluding particles and geometric distortion, as well as the excellent contrast, should make the extraction of particle information simpler and the subsequent data analysis more straightforward. More importantly, however, although data from the internal flow structure would be very useful, information from the riser wall is vital to validating mathematical models. 


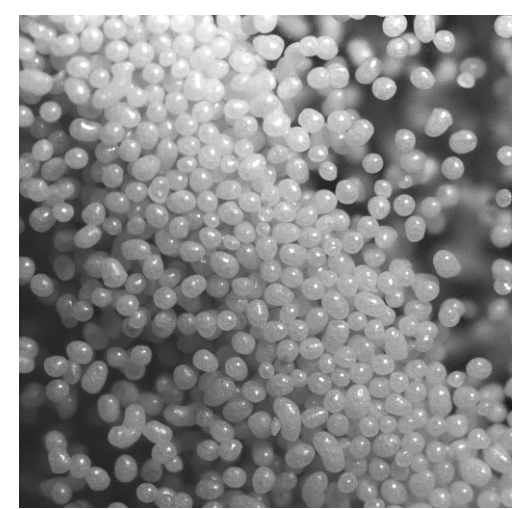

(a) Original image $I(x, y)$

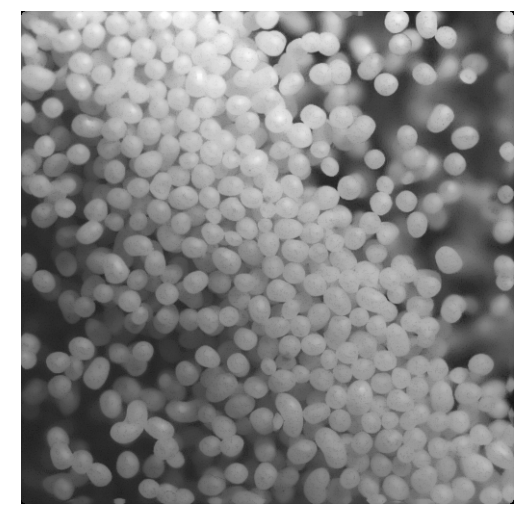

(c) Salt suppression $I(x, y)-I^{s}(x, y)$

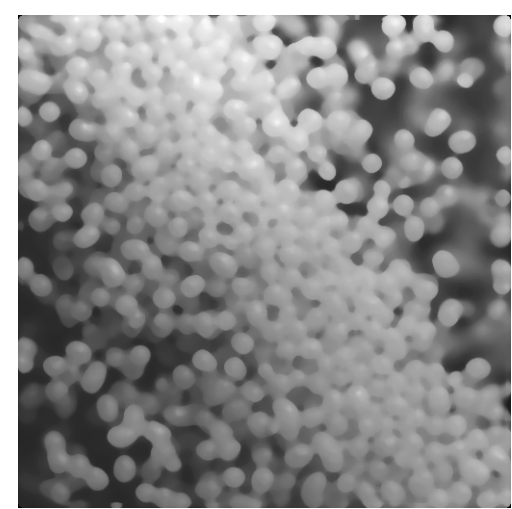

(b) Median filter output $I^{M}(x, y)$

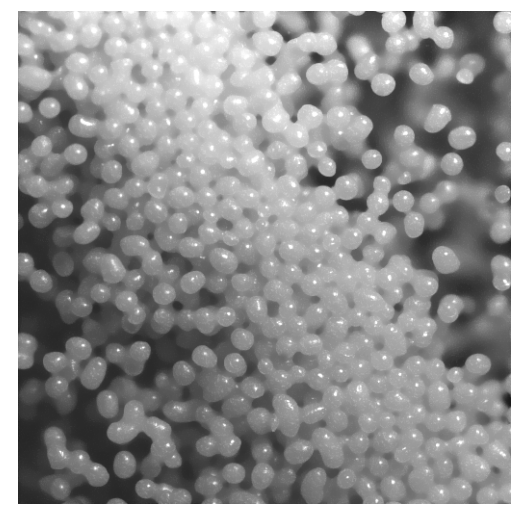

(d) Pepper suppression $I(x, y)-$ $I^{p}(x, y)$

Figure 2.13: Removing high-intensity (salt) and low-intensity (pepper) speckle noise. The median filter was applied with a $[9 \times 9]$ kernel.

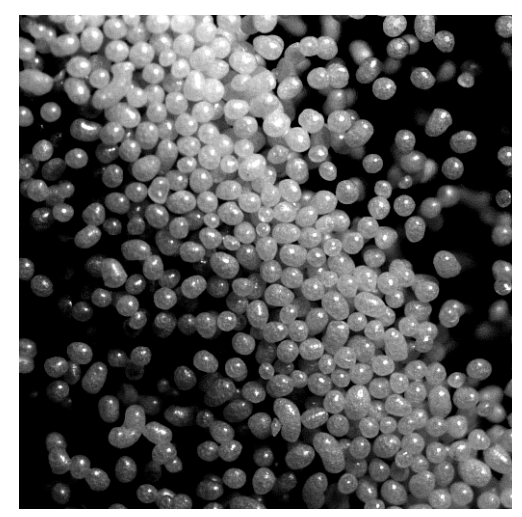

(a) Temporally adjusted image $I(x, y)$

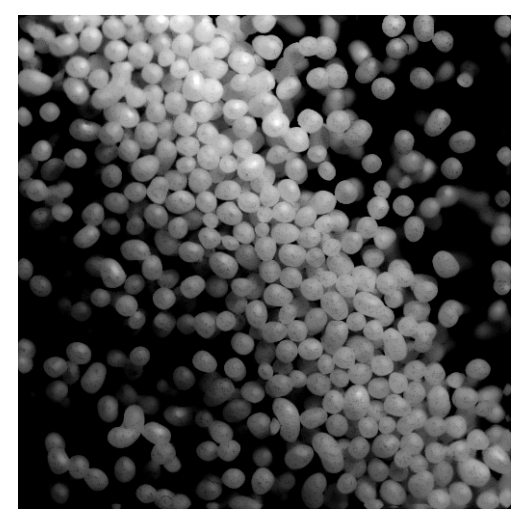

(b) Salt suppression $I(x, y)-I^{s}(x, y)$

Figure 2.14: Suppression of high-intensity speckle noise on a temporally adjusted image. 
This page is intentionally blank. 


\section{Chapter 3}

\section{Particle Detection}

\subsection{Introduction}

Images of the flow contain the two-dimensional projection of three-dimensional space due to the reflectance of light off particle matter [22]. The problem of determining the particle volume, a 3-dimensional measure, from flow images captured by a single camera is an ill-posed problem. However, if the particles lying on the focal-plane can be detected, then the problem of determining the particle area along the plane is trivial. If the total particle area can be extracted, equations exist in the literature to convert this estimated area to a volume $[25,46]$.

In order to determine the total area of the plane covered by particles for a single image, every particle on the focal-plane must be detected and their projected areas extracted. Summing all of these individual particle areas gives a total projected particle area $\mathcal{A}$. Thus, given $N_{p}$ elliptical bodies lying on the focal-plane, the total area is

$$
\begin{aligned}
\mathcal{A} & \triangleq \sum_{i=1}^{N_{p}} A_{i} \\
& =\sum_{i=1}^{N_{p}} \pi a_{i} b_{i},
\end{aligned}
$$

where $a_{i}$ and $b_{i}$ are the semi-major and semi-minor axes, respectively, of the $i^{\text {th }}$ particle. Considering an ellipse lying on the vertical image plane in 3-dimensional space, this projected area corresponds to the $x z$ plane. 
In calculating the properties of a flow, particles are typically defined as spheres given their mathematical simplicity [30]. For instance, in describing the size of a sphere the diameter alone is sufficient. When evaluating experimental data, however, non-spherical particles are often encountered. The geometry of these can be described in terms of an equivalent spherical diameter which, in terms of size, is simply the diameter of a sphere with an area equivalent to that of the particle [15].

Describing the area of each elliptical particle by an equivalent spherical diameter constrained to 2-dimensions gives

$$
\begin{aligned}
\mathcal{A} & =\sum_{i=1}^{N_{p}} \pi\left(\frac{d_{i}}{2}\right)^{2} \\
& =\frac{\pi}{4} \sum_{i=1}^{N_{p}} d_{i}^{2} .
\end{aligned}
$$

If the particle size distribution is monodisperse ${ }^{1}$ it seems reasonable to assume the equivalent spherical diameters $\left\{d_{i}\right\}_{i=1}^{N_{p}}$ are equal. Denoting the average equivalent particle diameter as $d_{p}$ gives

$$
\begin{aligned}
\mathcal{A} & =\frac{\pi}{4} \sum_{i=1}^{N_{p}} d_{p}^{2} \\
& =\frac{\pi}{4} N_{p} d_{p}^{2} .
\end{aligned}
$$

Thus, if the mean particle diameter $d_{p}$ is known and the total covered area of the image plane $\mathcal{A}$ can be estimated, then the particle count $N_{p}$ can be estimated by (3.6). This forms the basis of a set of experiments meant to evaluate the accuracy of particle detection schemes. Details of the experiments are given in the following section.

Instead of identifying the projected particle surfaces, as in determining $\mathcal{A}$, it may also be possible to identify the projected particle boundaries. Using the equivalent spherical diameters $\left\{d_{i}\right\}_{i=1}^{N_{p}}$ this would be equivalent to identifying the circumferences of circles. Given

\footnotetext{
${ }^{1} \mathrm{~A}$ monodisperse distribution has (nearly) a single particle size; approximately $\mathrm{CV}<10 \%$ [15]. CV is the coefficient of variation which is the standard deviation as a percentage of the mean [23], in this case in terms of the particle diameter.
} 
$N_{p}$ particles, define the total projected circumference as

$$
\begin{aligned}
\mathcal{C} & \triangleq \sum_{i=1}^{N_{p}} C_{i} \\
& =\sum_{i=1}^{N_{p}} \pi d_{i} .
\end{aligned}
$$

Under the assumption of a monodisperse distribution this reduces to

$$
\begin{aligned}
\mathcal{C} & =\sum_{i=1}^{N_{p}} \pi d_{p} \\
& =\pi N_{p} d_{p} .
\end{aligned}
$$

This suggests that $N_{p}$ can be estimated from the mean diameter and the total circumference of spherical particles.

The equivalent diameter $d_{p}$ is based on the area which has a nonlinear relationship with the circumference. Since a circle has the minimum boundary length for a given surface area, equating each particle to a circle of equivalent area will minimize the estimated total circumference $\mathcal{C}$. Therefore, to more accurately estimate $N_{p}$ by $\mathcal{C}$, the particles must be spherical. Note that if the particle count $N_{p}$ and the total circumference $\mathcal{C}$ are determined independently of each other, the ratio of the left and right-hand sides of (3.10) could be used to evaluate the sphericity of the particles where only true spherical particles would give a ratio of unity.

\subsection{Evaluation Method}

A set of sample images were selected to help evaluate the accuracy of possible detection schemes. The images were meant to cover the gamut of possible particle densities while remaining an ordered, sequential set. These images, having been part of a much larger sequence, are denoted $\left\{I_{1078+i}\right\}_{i=0}^{80}$. Since no baseline data or dependable detection method existed prior to this project, the analysis had to be completed manually. To reduce the number of images in the analysis and yet retain a good resolution in the extracted data, the set was subsampled such that every fifth frame was kept. The set of images $\mathcal{I}=\left\{I_{1078+5 i}\right\}_{i=0}^{16}$ that remained after subsampling are shown in Figures 3.1 and 3.2. 


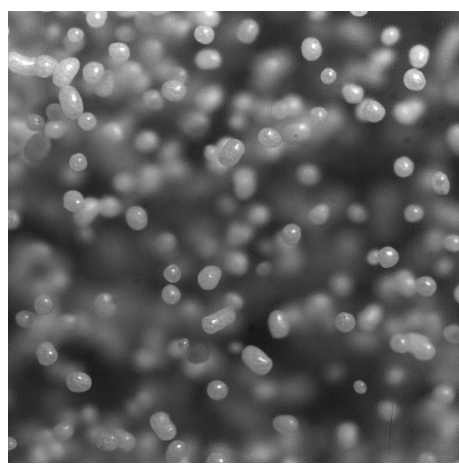

(a) 1078

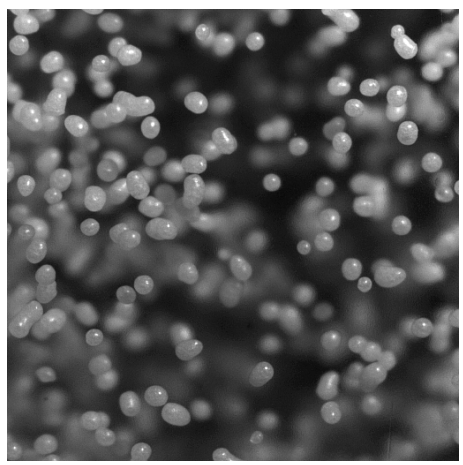

(d) 1093

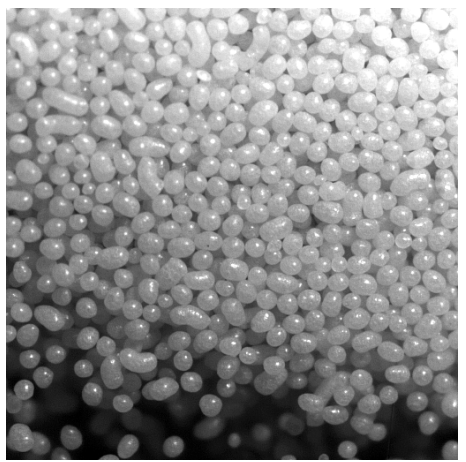

(g) 1108

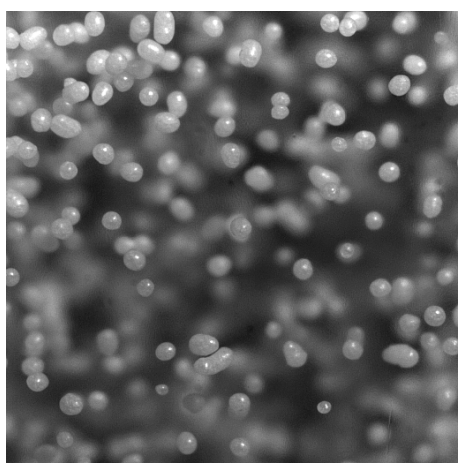

(b) 1083

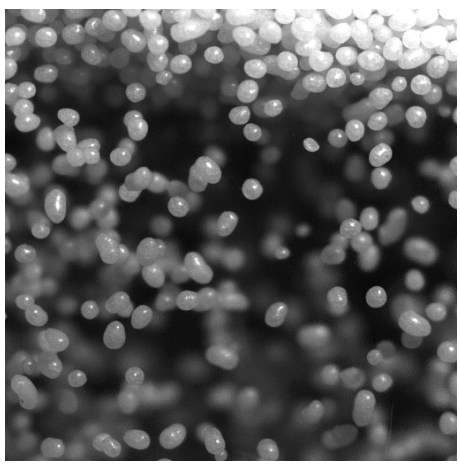

(e) 1098

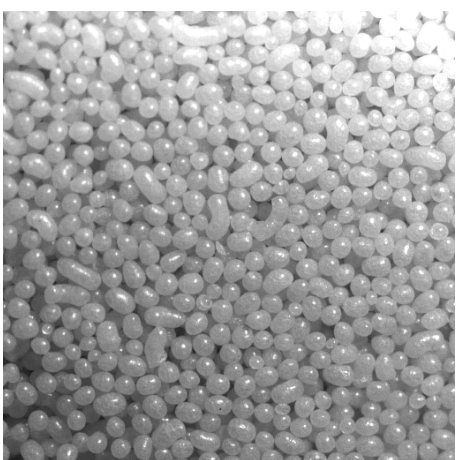

(h) 1113

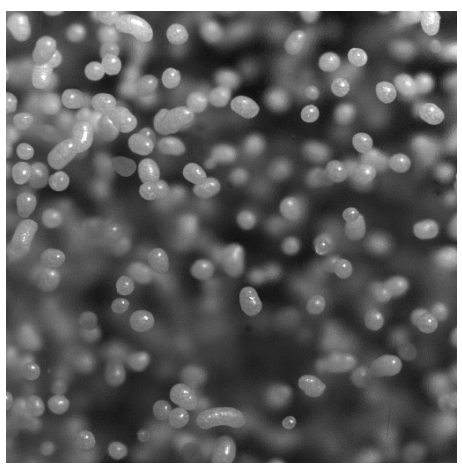

(c) 1088

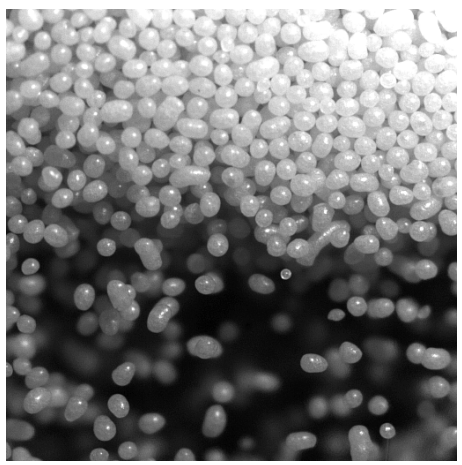

(f) 1103

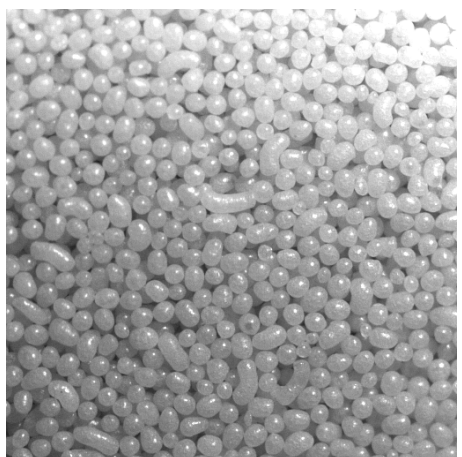

(i) 1088

Figure 3.1: Set of images $\left\{I_{1078+5 i}\right\}_{i=0}^{8}$ used to determine the baseline data. 


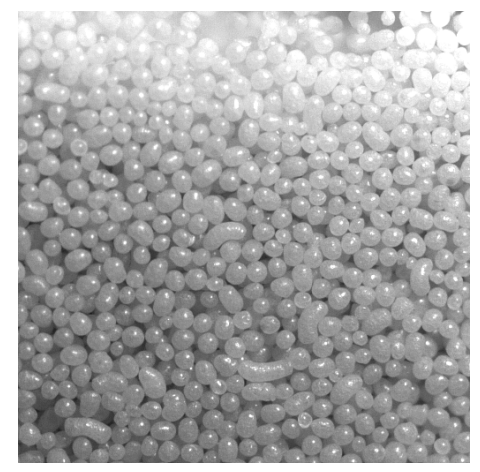

(a) 1123

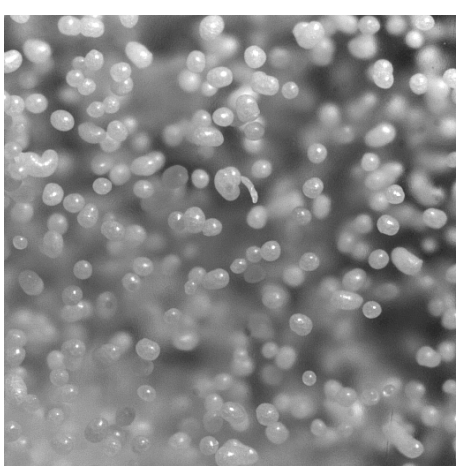

(d) 1138

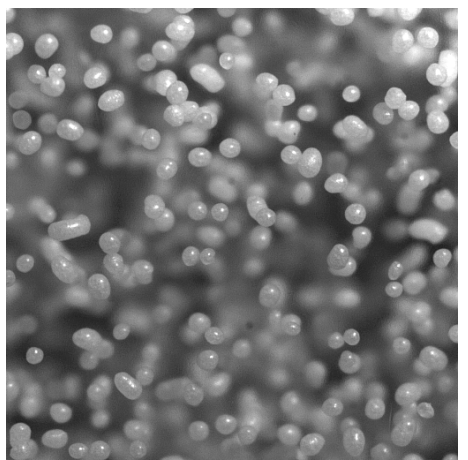

(g) 1153

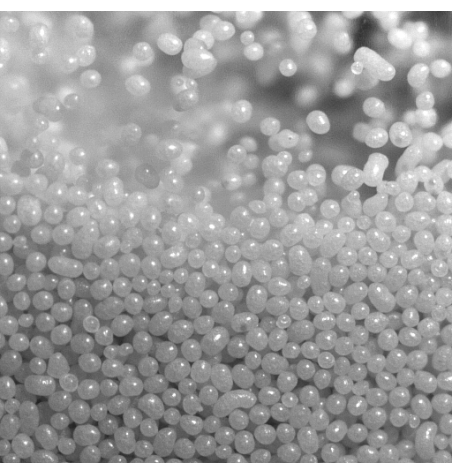

(b) 1128

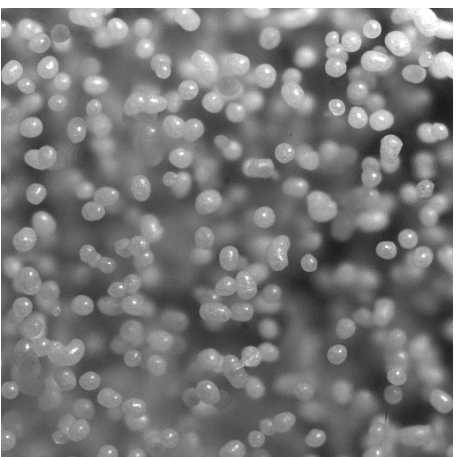

(e) 1143

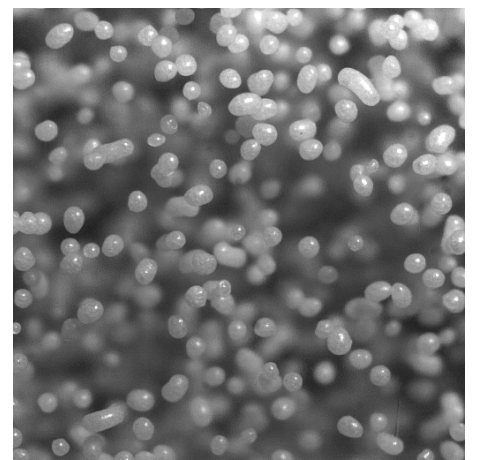

(h) 1158

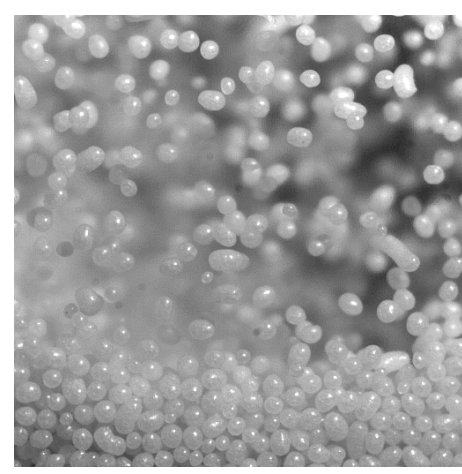

(c) 1133

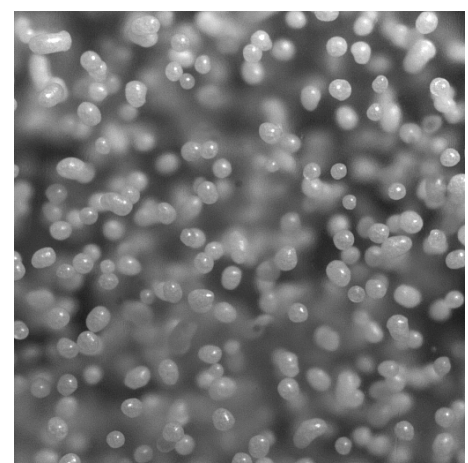

(f) 1148

Figure 3.2: Set of images $\left\{I_{1078+5 i}\right\}_{i=9}^{16}$ used to determine the baseline data. 
Any candidate detection scheme should determine the number of in-plane particles for a given image, either explicitly by counting or implicitly by first estimating $\mathcal{A}$ and then solving (3.6) for $N_{p}$. For the evaluation, the in-plane particles were manually counted in each image from $\mathcal{I}$. The resulting baseline for $\mathcal{I}$ is shown in Figure 3.3. The minimum was $N_{p}^{1078}=53$ and the maximum was $N_{p}^{1123}=432$ where $N_{p}^{k}$ denotes the particle count in image $I_{k}$.

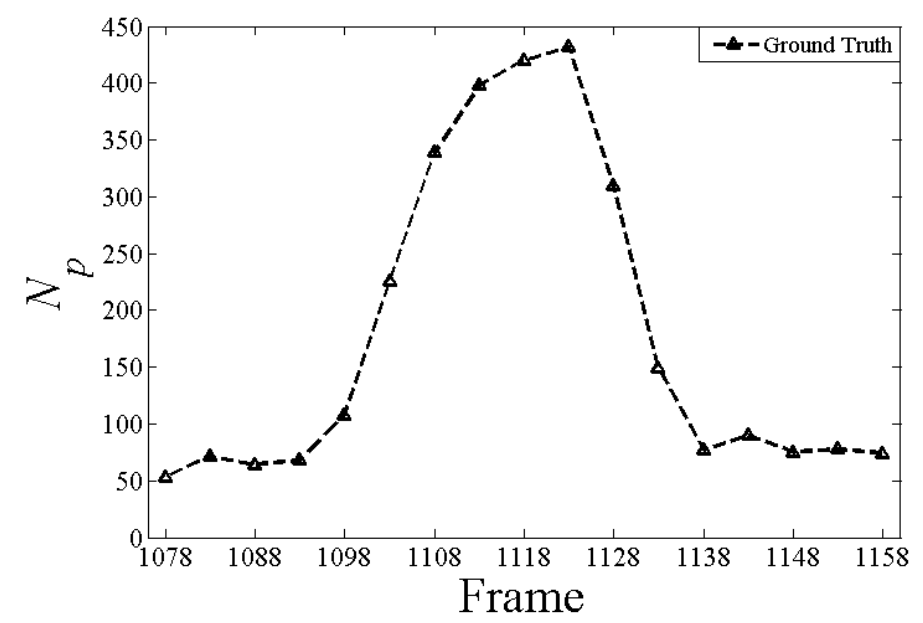

Figure 3.3: The ground truth (baseline) data manually extracted from the image sequence.

\subsection{Classification Techniques}

\subsubsection{Intensity-based Classification}

Each of the intensity images in this study can be defined as a set of pixels consisting of the union of three subsets,

$$
I=\mathcal{S}_{\mathcal{I}} \vee \mathcal{S}_{\mathcal{O}} \vee \mathcal{S}_{\mathcal{V}}
$$

where the subsets $\mathcal{S}_{\mathcal{I}}, \mathcal{S}_{\mathcal{O}}$, and $\mathcal{S}_{\mathcal{V}}$ are the pixels composing the set of in-focus particles, outof-focus particles, and the void (empty background), respectively. Identifying each subset could be beneficial, but the information required to determine the particle area $\mathcal{A}$, defined in the previous section, lies in $\mathcal{S}_{\mathcal{I}}$ alone. It would therefore be sufficient to identify the 
subsets $\mathcal{S}_{\mathcal{I}}$ and $\mathcal{S}_{\mathcal{B}}$, such that

$$
\begin{aligned}
\mathcal{S}_{\mathcal{B}} & =\mathcal{S}_{\mathcal{O}} \vee \mathcal{S}_{\mathcal{V}} \\
& =I \backslash \mathcal{S}_{\mathcal{I}} .
\end{aligned}
$$

By definition, $\mathcal{S}_{\mathcal{B}}$ is the subset of background pixels forming both the void and the out-offocus particles. By (3.13), identifying either subset $\mathcal{S}_{\mathcal{I}}$ or $\mathcal{S}_{\mathcal{B}}$ will serve to identify the other since $I$ is given.

For a digital image, if the set of pixels $\mathcal{S}_{\mathcal{I}}$ can be found, the cardinality of that set will be equivalent to $\mathcal{A}$. Thus,

$$
\begin{aligned}
\mathcal{A} & =\left|\mathcal{S}_{\mathcal{I}}\right| \\
& =\sum_{x \in \mathcal{X}} \sum_{y \in \mathcal{Y}} \delta_{I}(x, y),
\end{aligned}
$$

where $\mathcal{X}$ and $\mathcal{Y}$ are the image rows and columns, respectively, as defined in $\S 2.1 .1$, and $\delta_{I}(\cdot, \cdot)$ is an intensity space indicator function such that

$$
\delta_{I}(x, y)=\left\{\begin{array}{lll}
1 & \text { if } & I(x, y) \in \mathcal{S}_{\mathcal{I}} \\
0 & \text { if } & I(x, y) \notin \mathcal{S}_{\mathcal{I}}
\end{array}\right.
$$

In order to identify the pixels $\mathcal{S}_{\mathcal{I}}$, some type of decision making process is required. A number of techniques exist that attempt to segment an image into multiple pixel classes. The method to partition the pixels and the computational resources required depend on the technique as well as the images themselves. The experimental images are relatively large ${ }^{2}$, high-resolution images. They could be downsampled to reduce the computational overhead, but that could decrease the information content.

\section{Thresholding}

A computationally and conceptually simple method for classifying a set of values $\mathcal{S}$ into two disjoint subsets, $\mathcal{S}_{\alpha}$ and $\mathcal{S}_{\beta}$, is to compare each member of $\mathcal{S}$ to some threshold $T$. On one side of the threshold lie the values of $\mathcal{S}_{\alpha}$ and on other side the values of $\mathcal{S}_{\beta}$. For a grayscale image $I$ with intensities $I(x, y) \in \mathcal{R}$ and some threshold $T_{I}$ (the $I$ denotes a

\footnotetext{
${ }^{2}$ Externally captured images of the flow in the NETL CFB are typically [ $\left.512 \times 512\right]$.
} 
threshold in the intensity space) the decision rule for classifying pixels as $\mathcal{S}_{\mathcal{I}}$ or $\mathcal{S}_{\mathcal{B}}$ is stated as:

$$
\text { If } I(x, y)<T_{I} \text {, decide } \mathcal{S}_{\mathcal{B}} ; \text { otherwise decide } \mathcal{S}_{\mathcal{I}} \text {. }
$$

This decision is based on the assumption that in-focus particles will have a higher intensity since they are closer to the light source. Alternatively, the decision rule can be written more compactly as a binary test:

$$
I(x, y) \underset{\mathcal{S}_{\mathcal{I}}}{\stackrel{\mathcal{S}_{\mathcal{B}}}{\lessgtr}} T_{I}
$$

It can also be applied directly to the definition of the indicator function,

$$
\delta_{I}(x, y)=\left\{\begin{array}{lll}
1 & \text { if } & I(x, y)>T_{I} \\
0 & \text { if } & I(x, y)<T_{I}
\end{array}\right.
$$

making the determination of $\mathcal{A}$ a trivial evaluation of (3.15).

Determining the value of the threshold is the key component to this technique. A commonly used method is the Otsu thresholding technique. The underlying assumption used by the Otsu method is that the sample distribution is bimodal and that a threshold value can be determined which maximizes the variance between the two modes [45]. Figure 3.4 shows the effect of using the Ostu method to threshold an experimental and adjusted image $^{3}$.

Applying the Otsu thresholding method to the original image (Figure 3.4(b)) has eliminated most of the particle boundary information, especially in the dense regions. The result for the adjusted image (Figure 3.4(d)) retained more of the boundaries, but there was still a noticeable loss. Both results include particles that were partially classified as $\mathcal{S}_{\mathcal{I}}$ and partially $\mathcal{S}_{\mathcal{B}}$. In theory this behavior should be expected from even an accurate classifier. The particles are not flat and their movement is not restricted to the two dimensions captured by the image, thus their visible surface is not likely to be entirely in-plane. If the extraction of individual particle locations is required from the classification result, this effect could complicate recognition algorithms.

\footnotetext{
3 "Adjusted" here refers to the preprocessing step described in $§ 2.2 .2$ meant to reduce the effect of nonuniform illumination.
} 


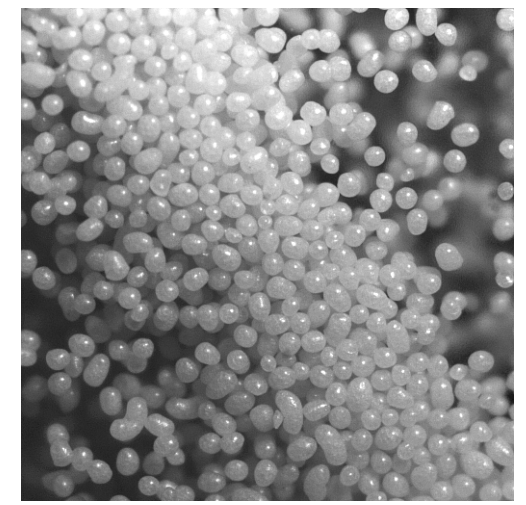

(a) Original: $I(x, y)$

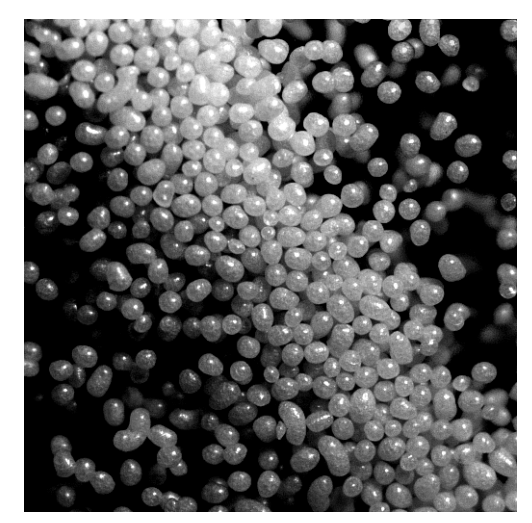

(c) Adjusted: $I^{\prime}(x, y)$

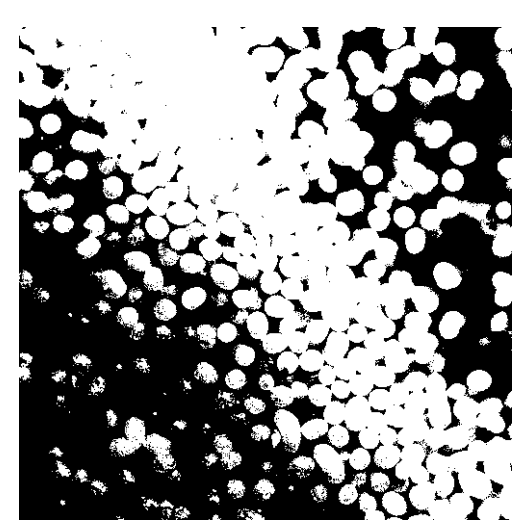

(b) $\delta_{I}(x, y) \mid T_{I}=0.5020$

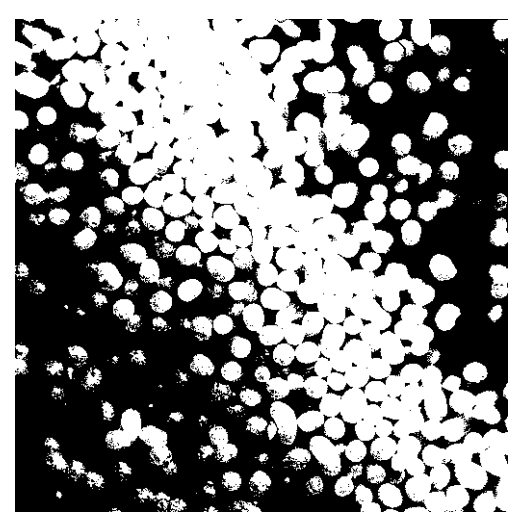

(d) $\delta_{I}(x, y) \mid T_{I}=0.3078$

Figure 3.4: Example of Otsu thresholding on an original image $I(x, y)$ and its adjustment result $I^{\prime}(x, y)=I(x, y)-I_{T}(x, y)$.

One possible solution is to pick a number of local thresholds across a single image. To do so, the image is split into a number of equal-sized blocks, or tiles, which are independently thresholded. Thus, for each tile, the Otsu threshold is determined and used to binarize only that tile. Results of this technique, performed on the images seen in Figures 3.4(a) and 3.4(c), are shown in Figures 3.5 and 3.6, respectively, using square tiles of size $\left\{\left[2^{i} \times 2^{i}\right]\right\}_{i=1}^{9}$. The images are $[512 \times 512]$ and $\log _{2} 512=9$, so applying a localized Otsu threshold on these images with a tile of size $\left[2^{9} \times 2^{9}\right]$ is the same as applying a single Otsu threshold to the image (Figure 3.4). For both image types, original and adjusted, the local method applied using tile sizes $[2 \times 2]$ through $[16 \times 16]$ appear very noisy. Artifacts of the tiling process are visible in the larger sizes as well, but the particle boundaries have mostly been preserved. The local implementation appears to be a significant improvement. 


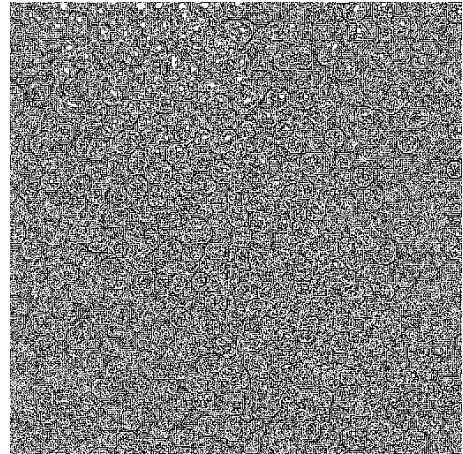

(a) $[2 \times 2](65536$ blocks $)$

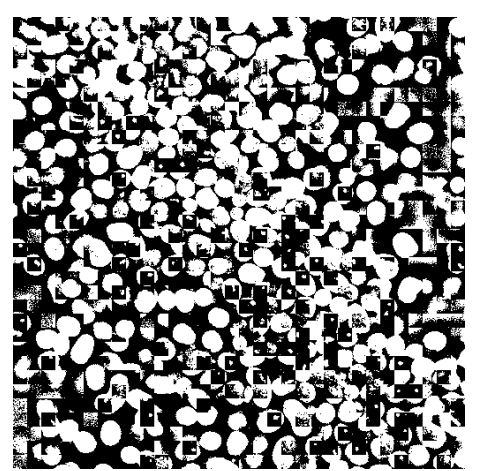

(d) $[16 \times 16](1024$ blocks $)$

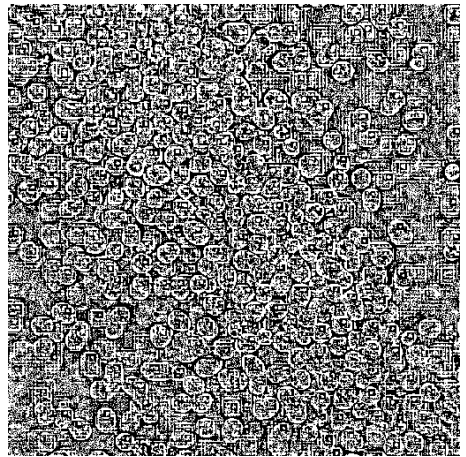

(b) $[4 \times 4](16384$ blocks $)$

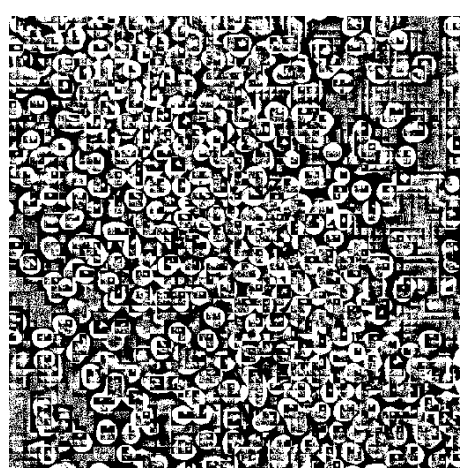

(c) $[8 \times 8](4096$ blocks $)$

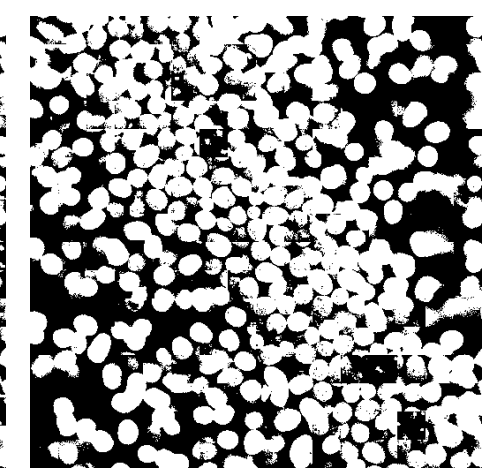

(e) $[32 \times 32](256$ blocks $)$

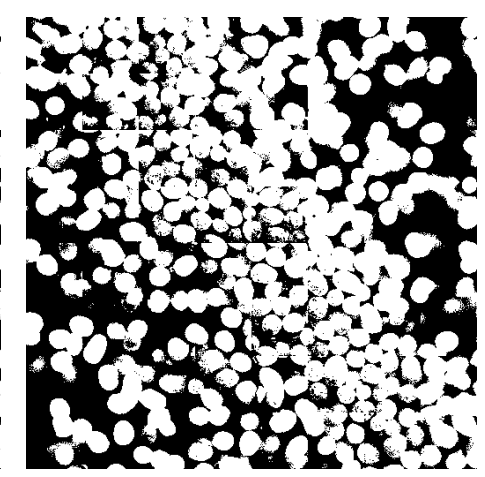

(f) $[64 \times 64](64$ blocks $)$

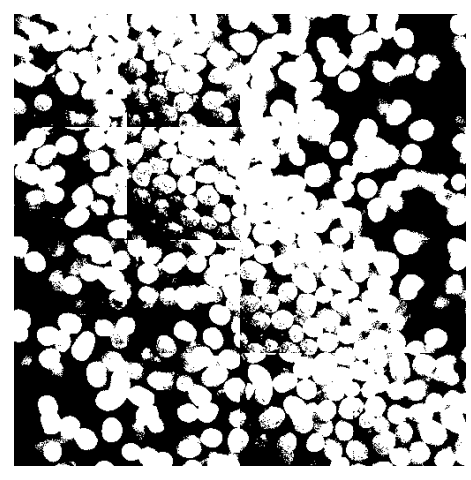

(g) $[128 \times 128](16$ blocks $)$

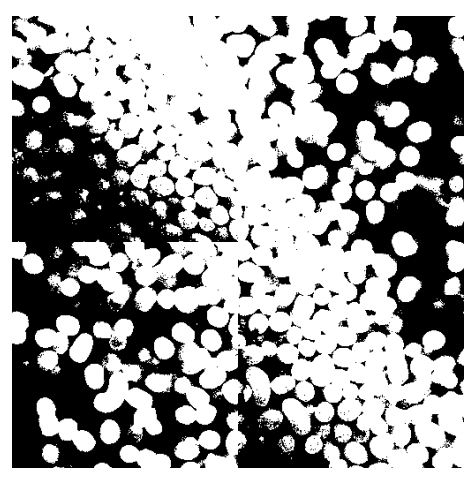

(h) $[256 \times 256](4$ blocks $)$

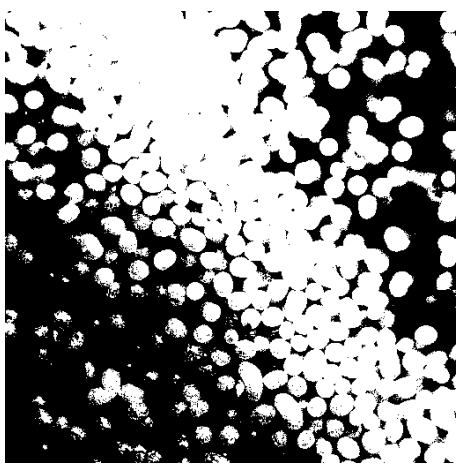

(i) $[512 \times 512](1$ block $)$

Figure 3.5: Localized Otsu thresholding applied to the original images using various block sizes. The image from Figure 3.4(a) has been used with tiles of size $\left\{\left[2^{i} \times 2^{i}\right]\right\}_{i=1}^{9}$. 


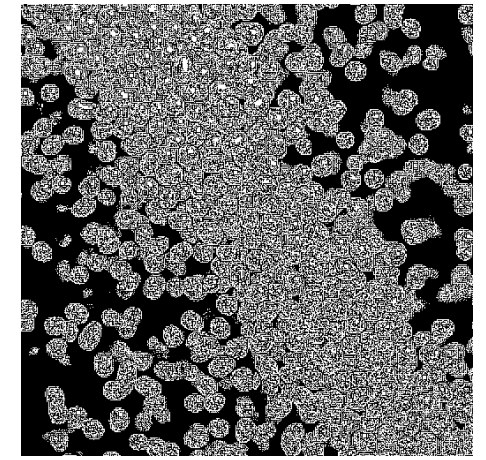

(a) $[2 \times 2](65536$ blocks $)$

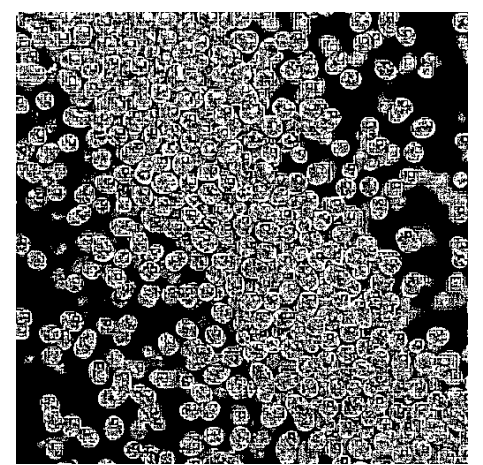

(b) $[4 \times 4](16384$ blocks $)$

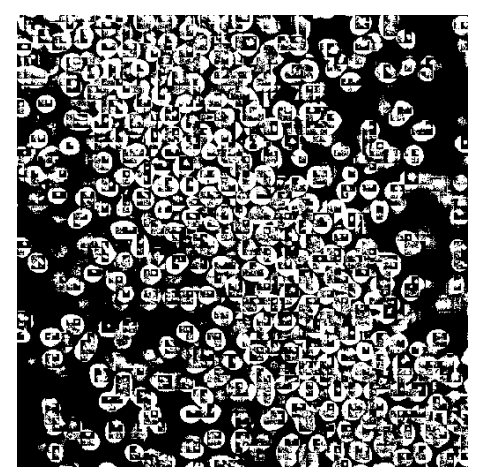

(c) $[8 \times 8](4096$ blocks $)$

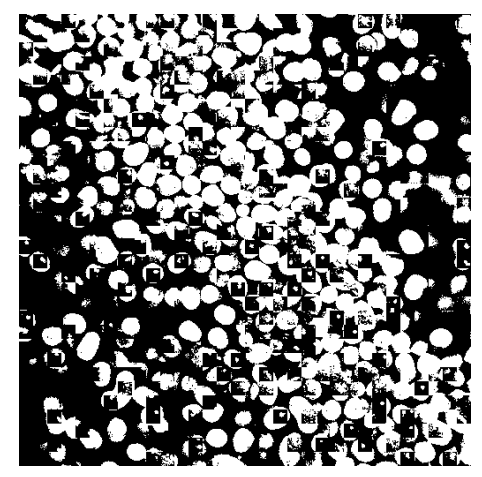

(d) $[16 \times 16](1024$ blocks $)$

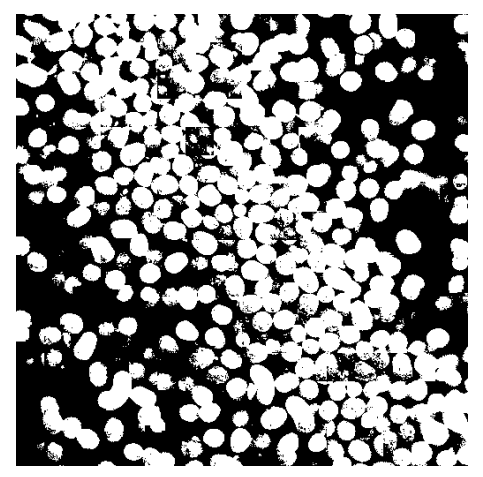

(e) $[32 \times 32](256$ blocks $)$

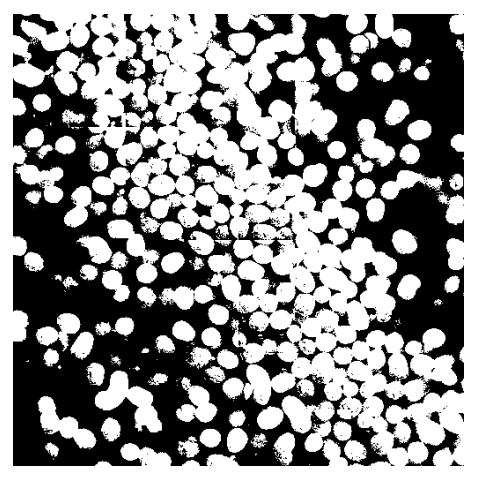

(f) $[64 \times 64](64$ blocks $)$

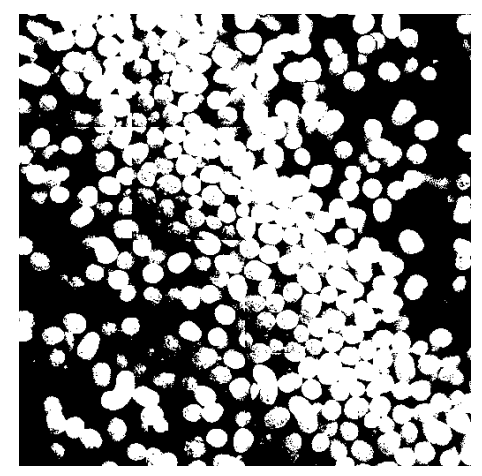

(g) $[128 \times 128](16$ blocks $)$

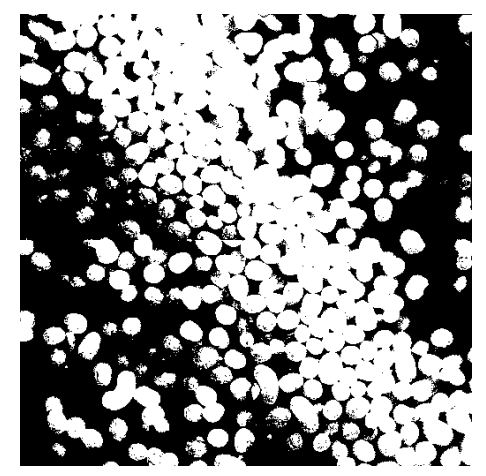

(h) $[256 \times 256](4$ blocks $)$

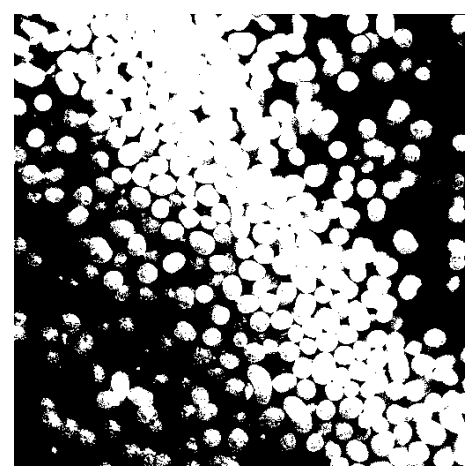

(i) $[512 \times 512](1$ block $)$

Figure 3.6: Localized Otsu thresholding applied to the temporally adjusted images using various block sizes. The image from Figure 3.4(c) has been used with tiles of size $\left\{\left[2^{i} \times\right.\right.$ $\left.\left.2^{i}\right]\right\}_{i=1}^{9}$. 


\section{Results}

Regardless of the specific thresholding technique, once the pixel classification has been completed, the particle count $N_{p}$ can be determined. Using (3.14) to define the area $\mathcal{A}$, combined with (3.6), the approximated particle count $\widehat{N}_{p}$ can be determined from

$$
\widehat{N}_{p}=\frac{4\left|\mathcal{S}_{\mathcal{I}}\right|}{\pi d_{p}^{2}}
$$

Results of the thresholding techniques in terms of the particle count for the entire set of images $\left\{I_{1078+i}\right\}_{i=0}^{80}$ are shown in Figure 3.7 alongside the baseline determined for $\mathcal{I}$. "Global Otsu" refers to a single threshold used for the entire image sequence and "Local Otsu[ $[M]$ " refers to the localized Otsu technique with tiles of size $[M \times M]$. The results for block sizes $[2 \times 2],[4 \times 4]$, and $[8 \times 8]$ were essentially noise signals, seen as relatively constant values across the middle of the plot, and were therefore not included.

Two error measures were used to evaluate the results. The first is the mean squared error (MSE) which is a fairly typical measure of the difference between two signals that can be defined using the $l_{2}$-norm (Euclidean distance) [60]. The $l_{2}$-norm is one of a family of metrics known as the Minkowski metric or the $l_{p}$-norm [17] and is denoted as:

$$
\begin{aligned}
l_{p}(\mathbf{a}, \mathbf{b}) & =\|\mathbf{a}-\mathbf{b}\|_{p} \\
& =\left(\sum_{i=1}^{N}\left|a_{i}-b_{i}\right|^{p}\right)^{\frac{1}{p}},
\end{aligned}
$$

where $\mathbf{a}$ and $\mathbf{b}$ are vectors. Let $\mathbf{g}$ be the vector of baseline (ground truth) values from $\mathcal{I}$ and $\hat{g}$ be the vector of estimated values for the same images. The mean squared error is then

$$
\begin{aligned}
E_{\mathrm{MSE}}(\mathbf{g}, \hat{\mathbf{g}}) & =\frac{1}{N_{f}}\left(l_{2}(\mathbf{g}, \hat{\mathbf{g}})\right)^{2} \\
& =\frac{1}{N_{f}}\left(\|\mathbf{g}-\hat{\mathbf{g}}\|_{2}\right)^{2} \\
& =\frac{1}{N_{f}} \sum_{i=1}^{N_{f}}\left(g_{i}-\hat{g}_{i}\right)^{2}
\end{aligned}
$$

where $N_{f}$ is the number of frames in the sequence. The MSE values for each method applied 


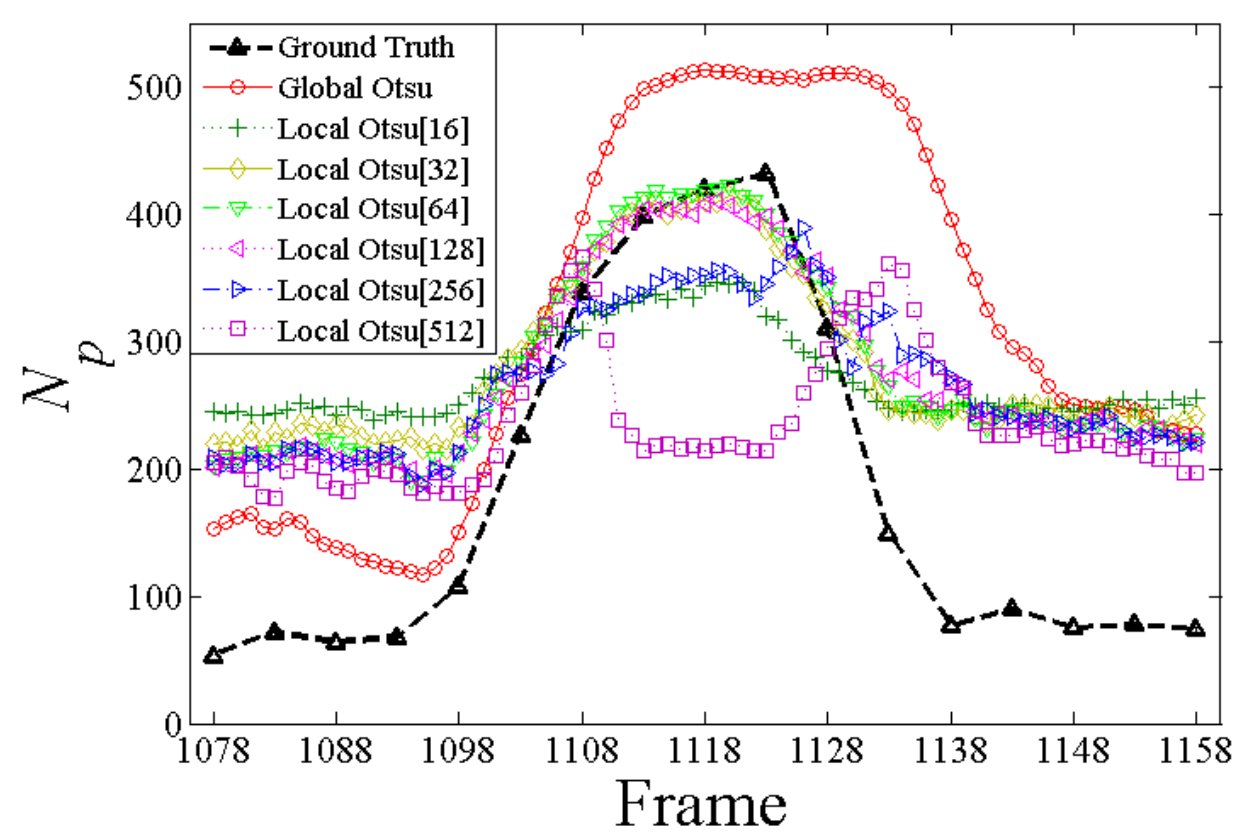

(a) Results on Original Images

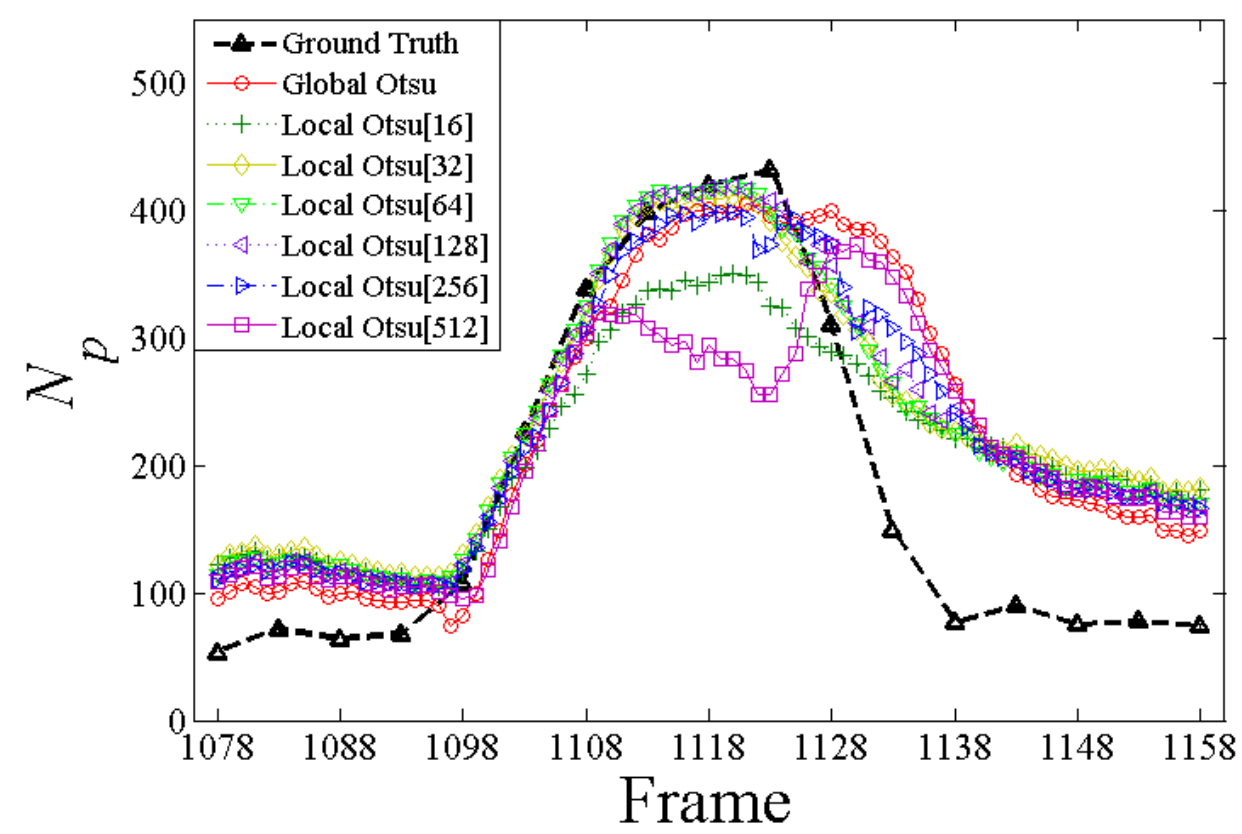

(b) Results on Temporally Adjusted Images

Figure 3.7: Particle detection results for variations of the Otsu thresholding method. 
to each image type are shown in Figure 3.8(a). Although estimates of $N_{p}$ were determined for the entire sequence $\left\{I_{1078+i}\right\}_{i=0}^{80}$, since only the results from $\mathcal{I}$ were used in calculating the error, the number of frames was $N_{f}=17$.

The second measure, the mean percentage error (MPE), is a linear estimate of the experimental error based on the normalized $l_{1}$-norm (Manhattan distance) and is defined as

$$
\begin{aligned}
E_{\mathrm{MPE}}(\mathbf{g}, \hat{\mathbf{g}}) & =\frac{1}{N_{f}}\left(l_{1}(\mathbf{g}, \hat{\mathbf{g}}) \cdot \frac{1}{\mathbf{g}}\right) \\
& =\frac{1}{N_{f}}\left(\|\mathbf{g}-\hat{\mathbf{g}}\|_{1} \cdot \frac{1}{\mathbf{g}}\right) \\
& =\frac{1}{N_{f}} \sum_{i}^{N_{f}} \frac{\left|g_{i}-\hat{g}_{i}\right|}{g_{i}} .
\end{aligned}
$$

Notice that each difference is normalized by the baseline data such that, when multiplied by 100 , it will represent the percentage error of the estimated value. The mean percentage error results are shown in Figure 3.8(b).

Both error measures show a large improvement when the adjusted images are used instead of the originals, suggesting the non-uniform illumination is detrimental to estimates of $N_{p}$ in the intensity space. While the adjusted images give improved estimates, the right tail of the concentration plot in Figure 3.7(b) is still poorly determined. This stems from the evaluation images themselves. The images from frame number 1128 through the end include a dense cloud of particles moving in the background. While these particles are far from the focal-plane, the high concentration of this cloud reflects a large amount of light to the camera, making an intensity-based classification difficult. Note that if the density at the wall (in-plane) is very high, the particles in the background would not be visible and would, therefore, not complicate the detection process.

\section{Dispersed versus Swarm Phases}

The baseline data can be divided into the dilute and dense concentration phases similar to Matsuda et al. [40]. The baseline data being used in the evaluation is about evenly split between a fairly steady dilute state, seen in the tails, and the passing of a dense particle swarm, seen as the parabolic structure in the middle. In [40] the distinction between phases was qualitative, but here the median baseline particle count $\tilde{g}$ was used. If 


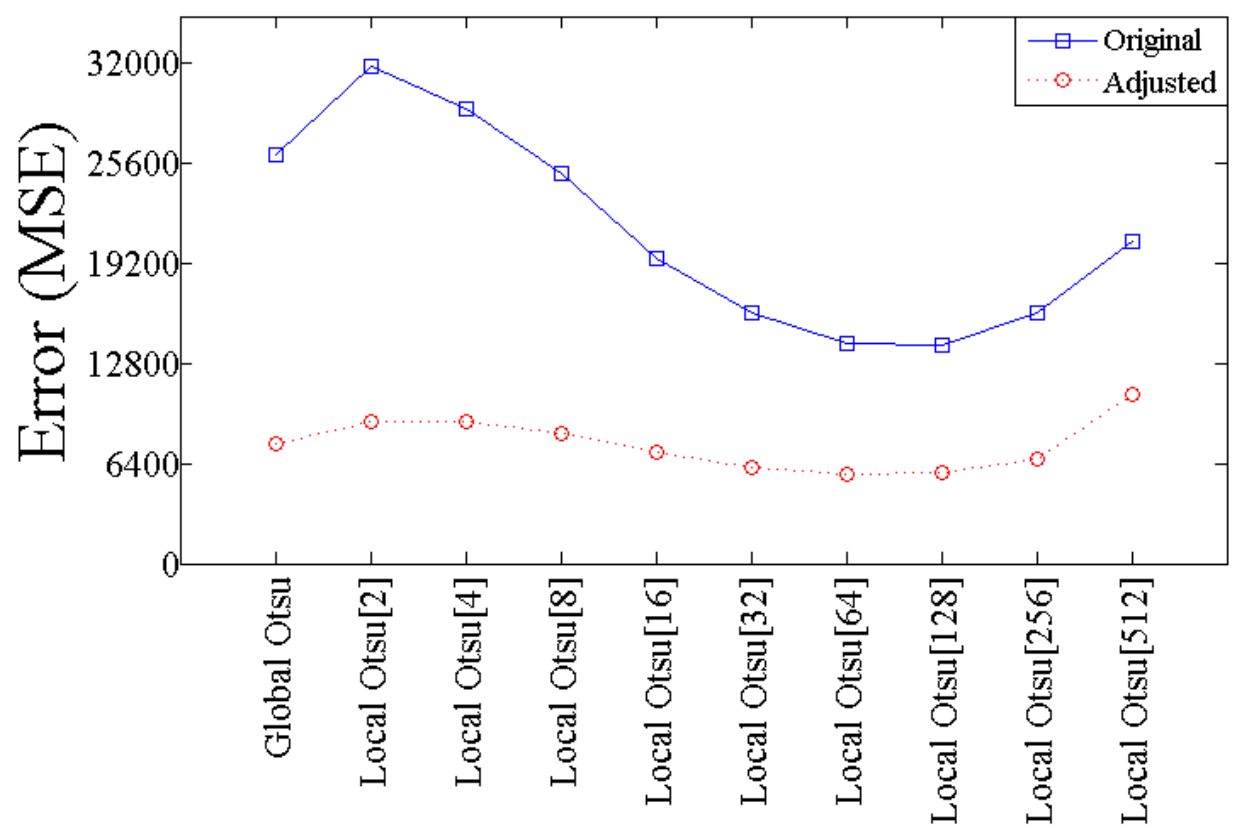

(a) Mean squared error

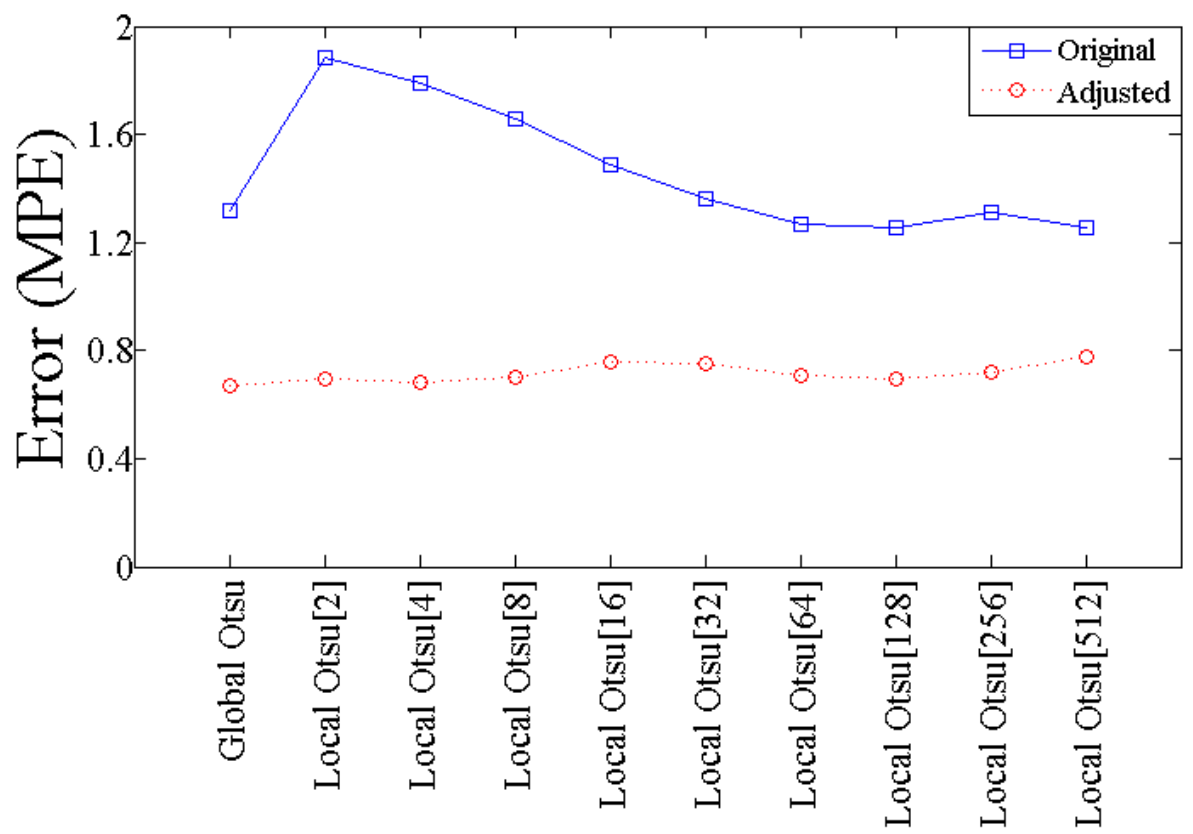

(b) Mean percentage error

Figure 3.8: Error values for intensity-based methods when compared to the ground truth. The techniques were evaluated on the original images as well as those adjusted to eliminate non-uniform illumination. 
$N_{p}^{i} \leq \tilde{g}$ then frame $I_{i}$ is considered to have captured the dilute phase whereas $N_{p}^{i}>\tilde{g}$ is indicative of the dense phase. For the set $\mathcal{I}$, the median value was $\tilde{g}=90$.

Estimates of the error were found for the dilute phase (dispersed) and dense phase (swarm) for all variations of the Otsu thresholding method considered in previous sections. The results, along with the overall MSE and MPE values, are shown in Figure 3.9. In each case thresholding on the adjusted images gave better results with the lowest errors given for the dense phase estimates. The minimum MPE and MSE were both given by the localized Otsu with blocks of size $[32 \times 32]$ on adjusted images in the dense phase $\left(E_{\mathrm{MPE}}=15.05 \%\right.$ and $E_{\mathrm{MSE}}=1816.2$ ).

Interestingly, the $[32 \times 32]$ blocks are just bigger than a particle of mean diameter $d_{p}=24$. Applying $[16 \times 16]$ blocks (see Figures $3.5(\mathrm{~d})$ and $3.6(\mathrm{~d})$ ) produced a number of particles that appear to have black boxes with a small white dot in them covering the surface. This was caused by the location of the block being completely (or at least mostly) within the boundary of the particle. The particle surfaces are fairly smooth other than the specular reflections, and thus the local threshold determined was very high, separating the highlight from the rest of the particle surface. This clearly suggests the block size should be larger than the particle.

\subsubsection{Gradient-based Classification}

A gradient image $G$ can be defined as a set consisting of the union of two subsets,

$$
G=\mathcal{S}_{\mathcal{E}} \vee \mathcal{S}_{\mathcal{B}}
$$

where the subsets $\mathcal{S}_{\mathcal{E}}$ and $\mathcal{S}_{\mathcal{B}}$ are the pixels forming edges and the background, respectively. If $G$ is such that the edges correspond to the particle boundaries then the cardinality of $\mathcal{S}_{\mathcal{E}}$ will be equivalent to $\mathcal{C}$, defined previously in $\S 3.1$. Thus,

$$
\begin{aligned}
\mathcal{C} & =\left|\mathcal{S}_{\mathcal{E}}\right| \\
& =\sum_{x \in \mathcal{X}} \sum_{y \in \mathcal{Y}} \delta_{G}(x, y),
\end{aligned}
$$




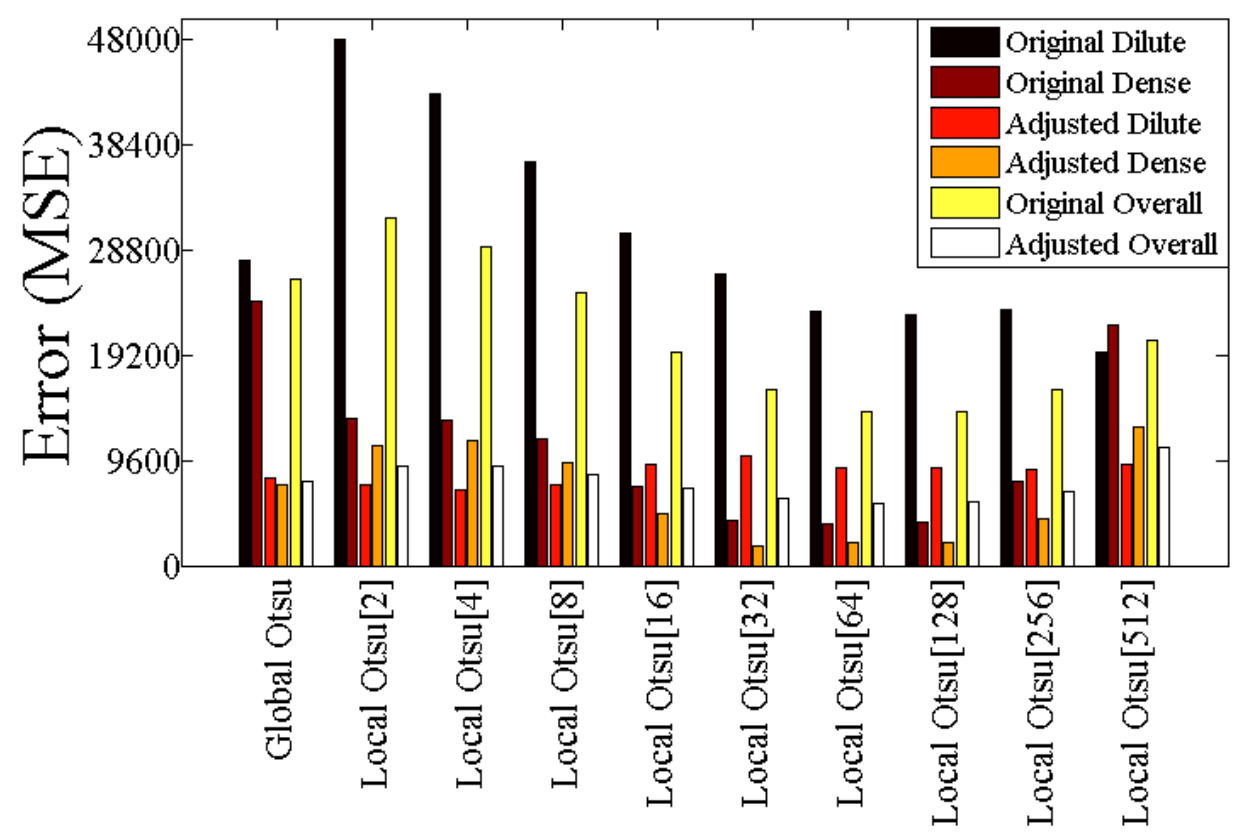

(a) Mean squared error

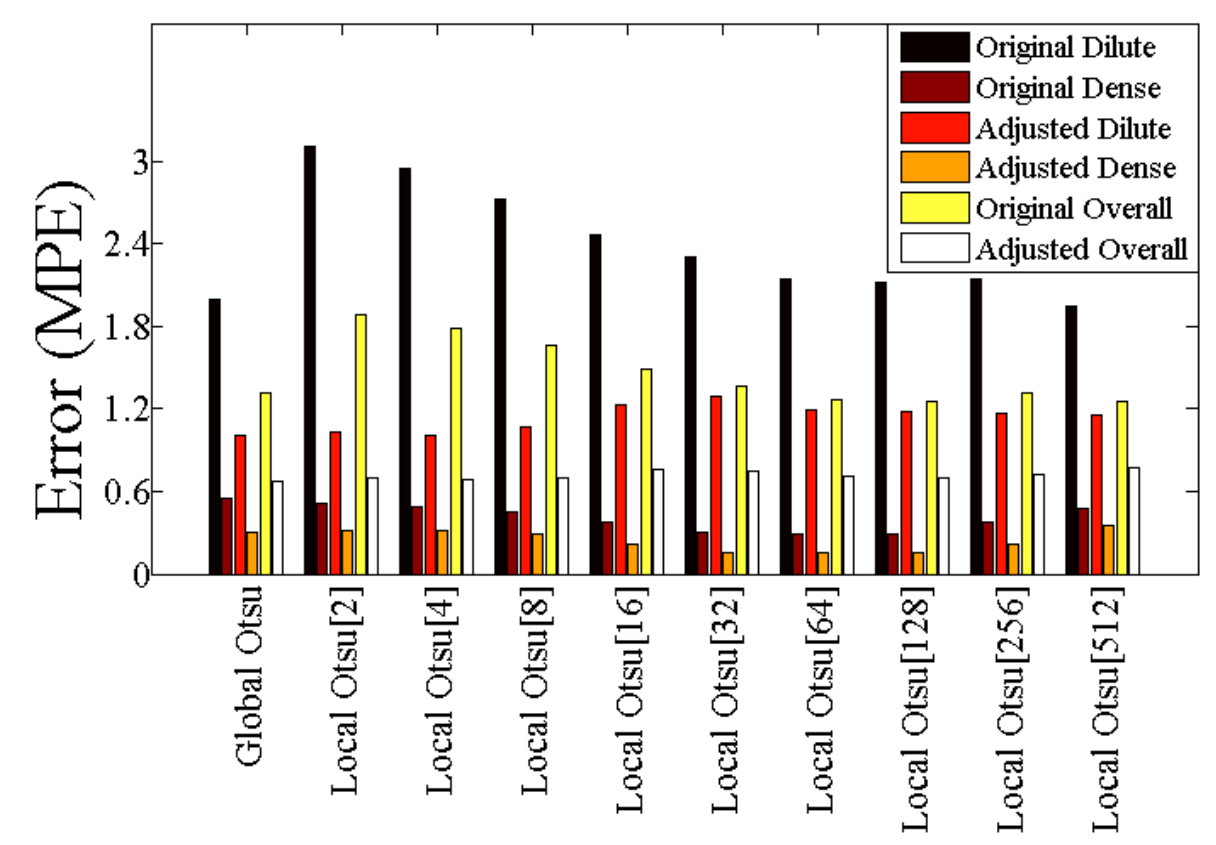

(b) Mean percentage error

Figure 3.9: Error values for intensity-based methods separated into dilute and dense concentration phases. Estimates of the error for the dilute phase, the dense phase, and overall are shown for both image types. 
where $\mathcal{X}$ and $\mathcal{Y}$ are the image rows and columns, respectively, and $\delta_{G}(\cdot, \cdot)$ is a gradient space indicator function such that

$$
\delta_{G}(x, y)=\left\{\begin{array}{lll}
1 & \text { if } & G(x, y) \in \mathcal{S}_{\mathcal{E}} \\
0 & \text { if } & G(x, y) \notin \mathcal{S}_{\mathcal{E}}
\end{array}\right.
$$

To identify the edge pixels $\mathcal{S}_{\mathcal{E}}$, an edge detection method is needed. A number of edge detection techniques exist ranging from very simple to very complex. Regardless of the technique, it is important to keep in mind the specular reflections on the particle surfaces. These areas have a very high intensity, often much brighter than the intensities of the surrounding surface. In the following discussion of gredient-based classification techniques, the images have been preprocessed to eliminate the specular highlights as described in $\S 2.3$. The evaluation of these techniques is similar to that done for the intensity-based techniques, the main difference being the use of the estimated circumference $\mathcal{C}$ to estimate $N_{p}$.

\section{Edge Filtering}

Edges are visually apparent when there is a large difference, or gradient, in the intensities of neighboring pixels. These gradients are found by determining the first derivatives of the pixel intensities in both the horizontal and vertical directions, since they can exist along any trajectory in 2-dimensional space. It is possible to approximate the derivatives simply by convolving the image with an appropriately defined mask $H$ [7]. Since the derivatives must be found in both the horizontal and vertical directions, two masks, $H_{x}$ and $H_{y}$, must be used. The masks $H_{x}$ and $H_{y}$ emphasize edges in the vertical and horizontal directions, respectively, since the edge will be normal to the gradient.

Approximations of the first derivative can be made using finite differences. The finite forward $\Delta^{+}$, backward $\Delta^{-}$, and central $\Delta^{c}$ differences are given as follows

$$
\begin{aligned}
\Delta^{+} f(x) & =\frac{f(x+\delta)-f(x)}{\delta} \\
\Delta^{-} f(x) & =\frac{f(x)-f(x-\delta)}{\delta} \\
\Delta^{c} f(x) & =\frac{f(x+\delta)-f(x-\delta)}{2 \delta}
\end{aligned}
$$

where $\Delta^{c} f=\Delta^{+} f+\Delta^{-} f$. Operating on the discrete grid of a digital image, the step size 
is usually $\delta=1$. The Roberts operators, shown in Figure 3.10, are produced by defining filters to find the backward differences along the $x$ and $y$ axes, but rotated by $\frac{\pi}{4}$. This rotation will actually emphasize diagonal edges, but reduces a location bias caused by the absence of a central pixel [7].

$$
\begin{array}{|c|c|}
\hline 0 & -1 \\
\hline 1 & 0 \\
\hline \text { (a) } H_{x}
\end{array} \quad \begin{array}{|c|c|}
\hline-1 & 0 \\
\hline 0 & 1 \\
\hline
\end{array}
$$

Figure 3.10: Roberts operators

One problem with finding finite differences is their high sensitivity to noise. This can be reduced, however, by smoothing in the direction orthogonal to the difference [7]. The central difference in the $x$ direction can be found by convolving an image with $h_{c}=$ $\left[\begin{array}{lll}-1 & 0 & 1\end{array}\right]$. Smoothing in the normal direction can be achieved by convolving the image with $h_{s}=\left[\begin{array}{lll}1 & 1 & 1\end{array}\right]^{T}$. Multiplying the two together as an outer product gives

$$
\begin{aligned}
H_{x} & =h_{s} h_{c} \\
& =\left[\begin{array}{l}
1 \\
1 \\
1
\end{array}\right]\left[\begin{array}{lll}
-1 & 0 & 1
\end{array}\right] \\
& =\left[\begin{array}{lll}
-1 & 0 & 1 \\
-1 & 0 & 1 \\
-1 & 0 & 1
\end{array}\right]
\end{aligned}
$$

which is the basis of the Prewitt operators shown in Figure 3.11. The Sobel operators, shown in Figure 3.12, were found similarly but the smoothing kernel was $h_{s}=\left[\begin{array}{lll}1 & 2 & 1\end{array}\right]^{T}$ which provides a little more smoothing than with the Prewitt operators [7].

\begin{tabular}{|l|l|l|}
\hline-1 & 0 & 1 \\
\hline-1 & 0 & 1 \\
\hline-1 & 0 & 1 \\
\hline \multicolumn{3}{|c|}{ (a) $H_{x}$} \\
\hline
\end{tabular}

\begin{tabular}{|c|c|c|}
\hline-1 & -1 & -1 \\
\hline 0 & 0 & 0 \\
\hline 1 & 1 & 1 \\
\hline \multicolumn{3}{|c|}{ (b) $H_{y}$}
\end{tabular}

Figure 3.11: Prewitt operators

Convolving the image $I$ with the directional kernels $H_{x}$ and $H_{y}$ gives the gradient 


\begin{tabular}{|l|l|l|}
\hline-1 & 0 & 1 \\
\hline-2 & 0 & 2 \\
\hline-1 & 0 & 1 \\
\hline
\end{tabular}

(a) $H_{x}$

\begin{tabular}{|c|c|c|}
\hline-1 & -2 & -1 \\
\hline 0 & 0 & 0 \\
\hline 1 & 2 & 1 \\
\hline
\end{tabular}

(b) $H_{y}$

Figure 3.12: Sobel operators

response in those directions

$$
\begin{aligned}
& G_{x}=I \otimes H_{x}, \\
& G_{y}=I \otimes H_{y},
\end{aligned}
$$

where " $\otimes$ " denotes discrete convolution. The directional information can be combined to form the gradient magnitude image $G$ whose local maxima should coincide with strong edges, thus

$$
\begin{aligned}
G & =\|\nabla I\|_{2} \\
& =\sqrt{G_{x}^{2}+G_{y}^{2}}
\end{aligned}
$$

where $\nabla$ is the gradient operator.

\section{Thresholding}

Once the gradient image $G$ has been determined it must be thresholded, much like the intensity image in $§ 3.3 .1$, to identify the pixels corresponding to particle boundaries. Results of the Sobel edge detector are shown in Figure 3.13 where the threshold $T_{G}$ (the $G$ denoting its use in the gradient space) was determined using the Otsu method. Examples are given with and without applying specular highlight suppression (SHS). Notice that the highlights, when ignored, have high intensities in the gradient magnitude image and are thus well preserved in the thresholded result.

\section{Edge Thinning}

Thinning is a widely used image processing technique often applied to binary images for compression as well as improving the analysis of line patterns or contour shapes [33]. The method employed was a parallel algorithm described in [33] that requires two indepen- 


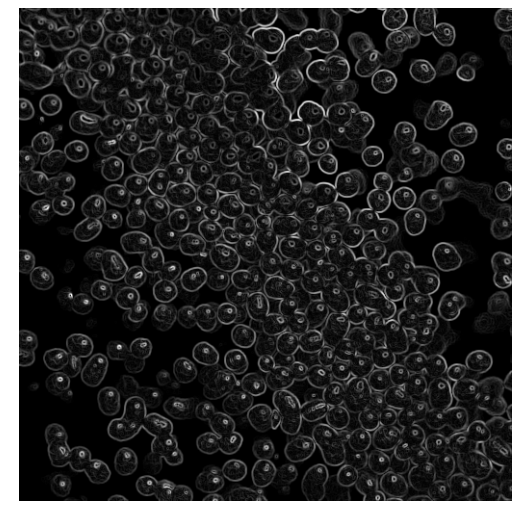

(a) Sobel gradient image $G(x, y)$

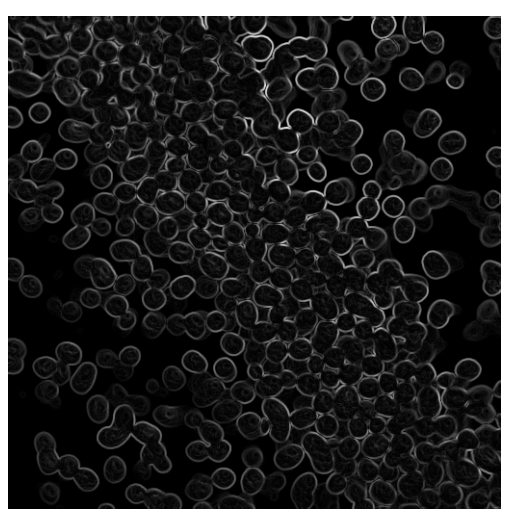

(c) Sobel gradient image $G(x, y)$ with SHS

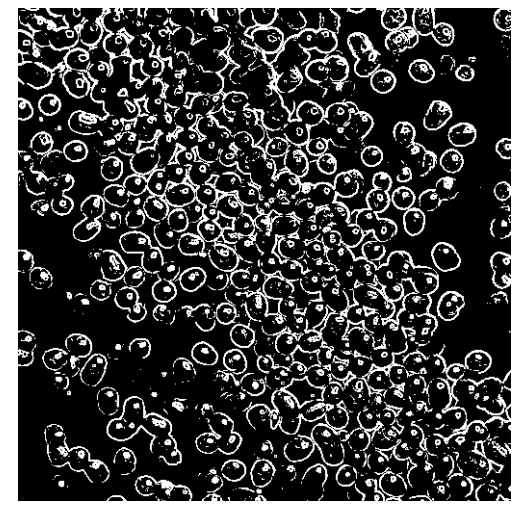

(b) $\delta_{G}(x, y) \mid T_{G}=0.0784$

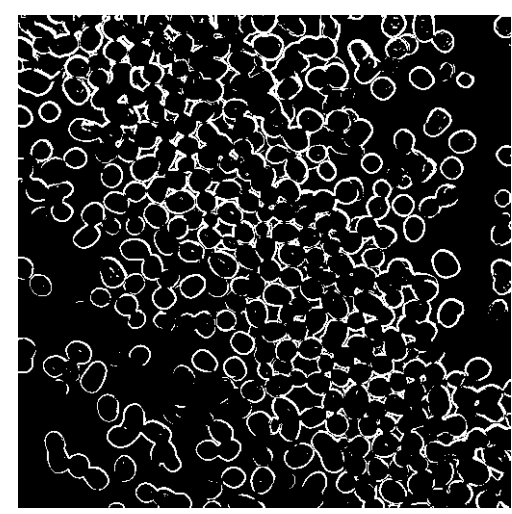

(d) $\delta_{G}(x, y) \mid T_{G}=0.0667$ with SHS

Figure 3.13: Example of gradient approximation and thresholding. The image from Figure 3.4(c) has been used with and without suppressing the specular highlights.

dent passes across the image. In each pass, marked pixels are tested against 3 criteria (slightly different for each) based on their $[3 \times 3]$ 8-connected neighborhood. The separate passes are required to correctly thin line segments while ensuring that any isolated $[2 \times 2]$ patches of marked pixels are retained [33].

Let $R_{1}$ and $R_{2}$ be binary images resulting from the two independent passes. In both images $R_{k}$, for $k \in\{1,2\}$, marked pixels are those flagged for removal in pass $k$. Once both passes are complete, the new thinned image is defined as

$$
I^{\prime}=I-\left(R_{1} \wedge R_{2}\right)
$$

Results of applying this thinning operation to the images shown in Figures 3.13(b) and 
3.13(d) are shown in Figures 3.14(a) and 3.14(b), respectively. Referring specifically to the result on the image with suppressed specularities, Figure 3.14(b), most of the boundary between two particles is lost if they are touching. This was a result of the gradient information itself and will surely add to the error of the estimate of $\mathcal{C}$.

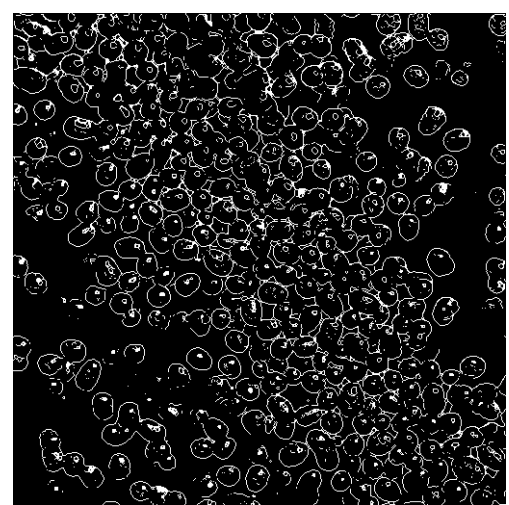

(a) Thinned $\delta_{G}(x, y)$

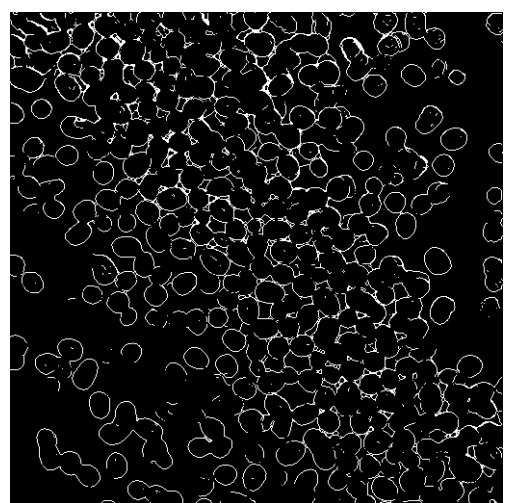

(b) Thinned $\delta_{G}(x, y)$ with SHS

Figure 3.14: Example of edge thinning. The binary images from Figure 3.13 have been used.

\section{Canny Edge Detector}

Another edge detection method is the Canny edge detector. Prior to determining the intensity gradients, the input image is convolved with a Gaussian mask to suppress noise [11]. Originally, the gradient image $G$ was found using finite forward differences in the form

$$
\begin{aligned}
& H_{x}=\left[\begin{array}{cc}
-1 & 1 \\
-1 & 1
\end{array}\right] \text { and } \\
& H_{y}=\left[\begin{array}{cc}
-1 & -1 \\
1 & 1
\end{array}\right],
\end{aligned}
$$

but the more robust finite central difference can be applied in both directions as simply $h_{c}$ and $h_{c}^{T}$ since the initial Gaussian smoothing eliminates the need for the added support of the Prewitt and Sobel operators [19, 7].

Using the directional gradients $\left[G_{x} G_{y}\right]^{T}$ formed by convolving the smoothed image $I_{\sigma}$ with $\left[\begin{array}{ll}H_{x} & H_{y}\end{array}\right]^{T}$, the gradient magnitude image is determined as before and the gradient 
directions are found by

$$
\begin{aligned}
\Theta(x, y) & =\angle G(x, y) \\
& =\tan ^{-1}\left(\frac{G_{y}(x, y)}{G_{x}(x, y)}\right) .
\end{aligned}
$$

The orientations $\Theta$ are used to drive non-maxima suppression in $[3 \times 3]$ neighborhoods along the gradient direction which effectively thins the gradients to single-pixel wide linesegments $[11,7]$.

Two thresholds $T_{\text {high }}$ and $T_{\text {low }}$ are then used to produce the final edge map. All gradient magnitudes larger than $T_{\text {high }}$ are retained as well as the pixels from connected candidate line-segments whose gradient magnitudes lie above $T_{\text {low }}[11]$. This form of hysteresis reduces the problem of broken edge contours common to edge detectors that apply gradient thresholds [11].

\section{Results}

Since each method of finding the edge map depended a great deal on the gradient threshold(s), a series of tests were conducted to find the best value(s) based on minimizing the MSE and the MPE for each method. The edge map was found for a range of gradient thresholds for each method and the subsequent error determined. Figure 3.15 shows the results of the test for the Roberts, Prewitt, and Sobel methods using the MPE metric on the original images. A similar test was completed for the Canny detector, although the high and low thresholds were both found. The test results for each type of image and each type of error metric had a similar shape, appearing as an exponential decay down to a global minima and then rising to a steady state.

Figure 3.16 shows the results of the edge detection methods using the thresholds found to minimize the MPE. None of the methods accurately approximate the particle count in the dense phase, although they appear to do very well in the dilute phase. The problem with the dense phase is likely the result of particles touching and any gradient information between their boundaries being lost as was seen in earlier examples. The closeness with which the dense phase is estimated, when particles are well separated, could imply that the assumption of spherical particles is a good one given the properties of $\mathcal{C}$ discussed earlier.

The MPE was determined for each edge detection method applied to both image types using both sets of thresholds: those that minimized the MSE and those that minimized 


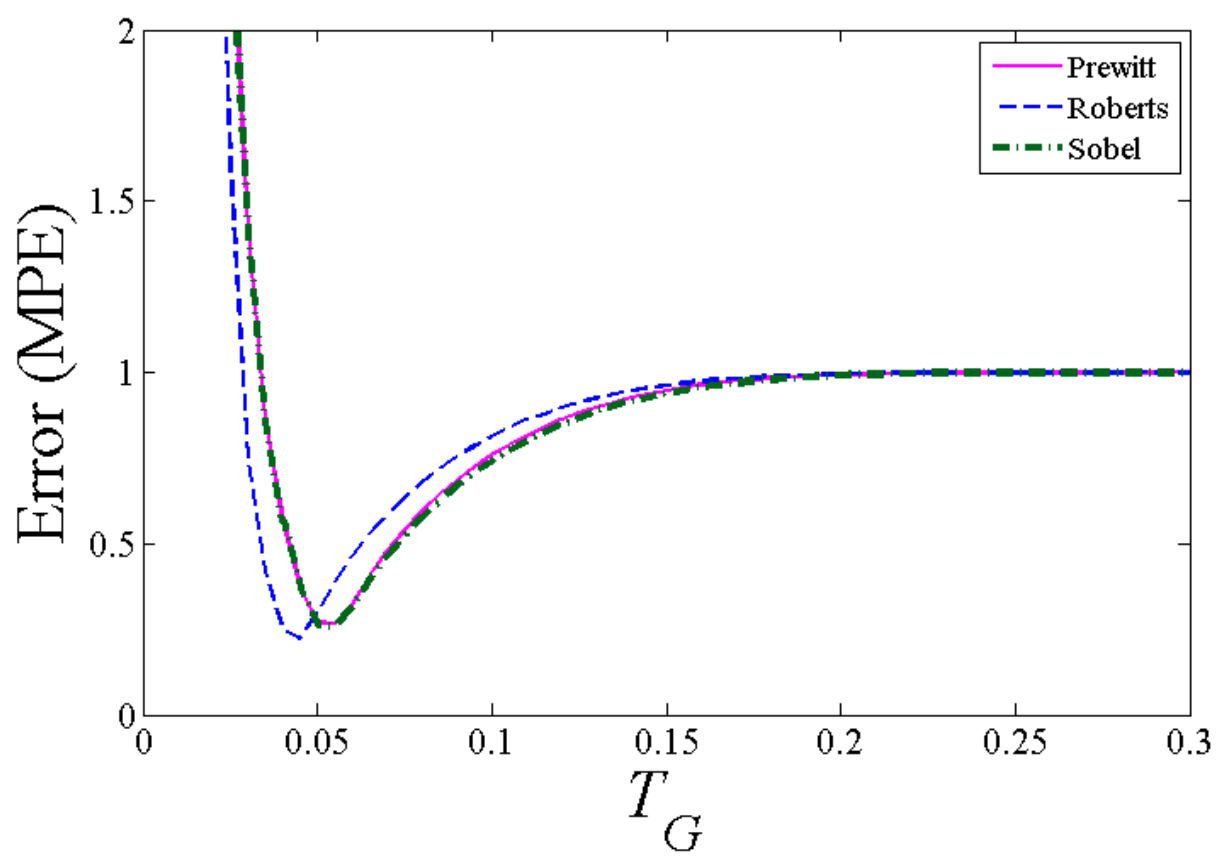

Figure 3.15: MPE error values for estimating the particle count based on various gradient thresholds.

the MPE. The results are shown in Figure 3.17. Interestingly the Roberts detector, the simplest in the group, outperformed the other methods $75 \%$ of the time.

\subsubsection{Learning-based Classification}

Instead of relying on the information of a single pixel alone, as with the intensity and gradient-based techniques already discussed, multiple features from a local neighborhood can be used together. Pattern classification techniques can be grouped into two types. The first, supervised classification, classifies patterns based on training data where the classes are known. The simplest is a one-nearest neighbor decision rule (1-NN) classifier which gives reasonable performance in most applications [29]. The second type, unsupervised classification, clusters groups of points into unlabeled classes in multi-dimensional space [29]. One of the most basic and computationally efficient algorithms is the $k$-means clustering algorithm [29]. The $k$-NN classifier and the $k$-means clustering algorithm, along with a decision rule for determining the class of each cluster, were both applied to the particle images. 


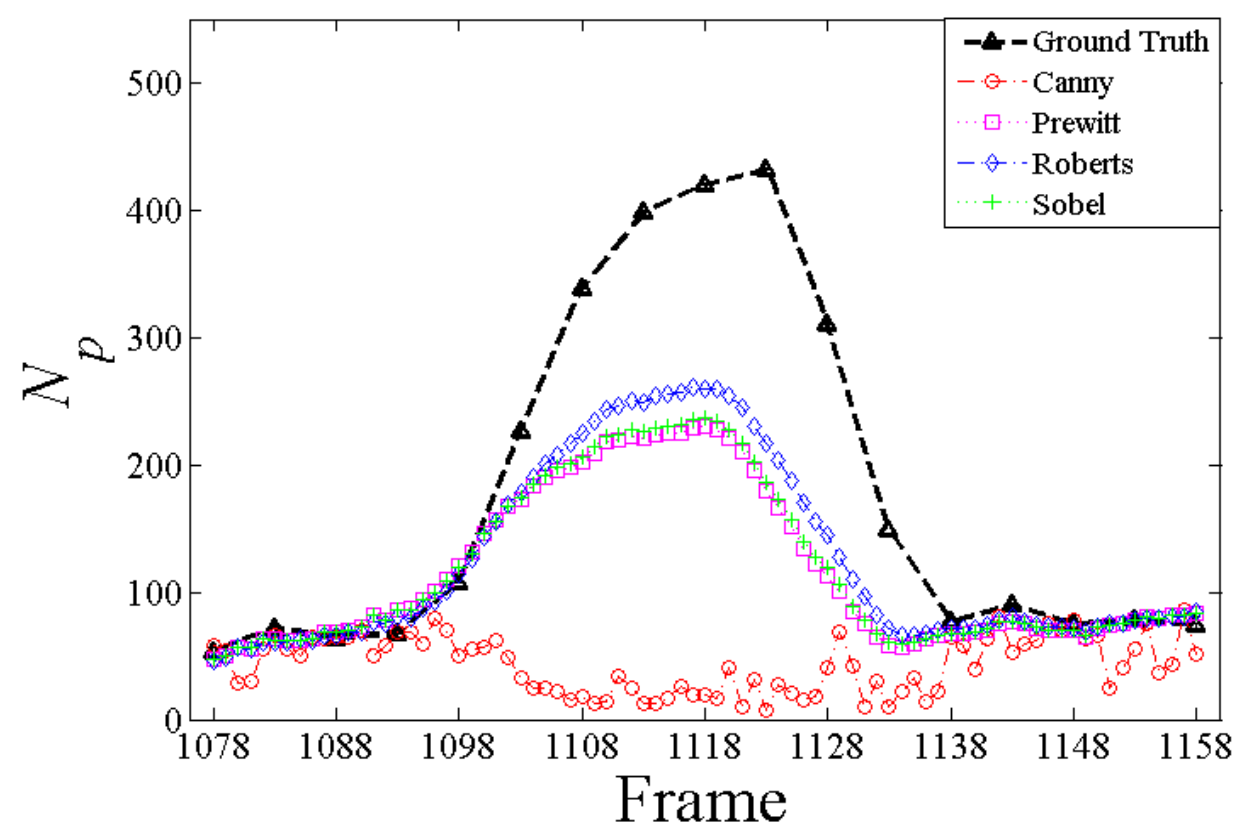

(a) Results on original images

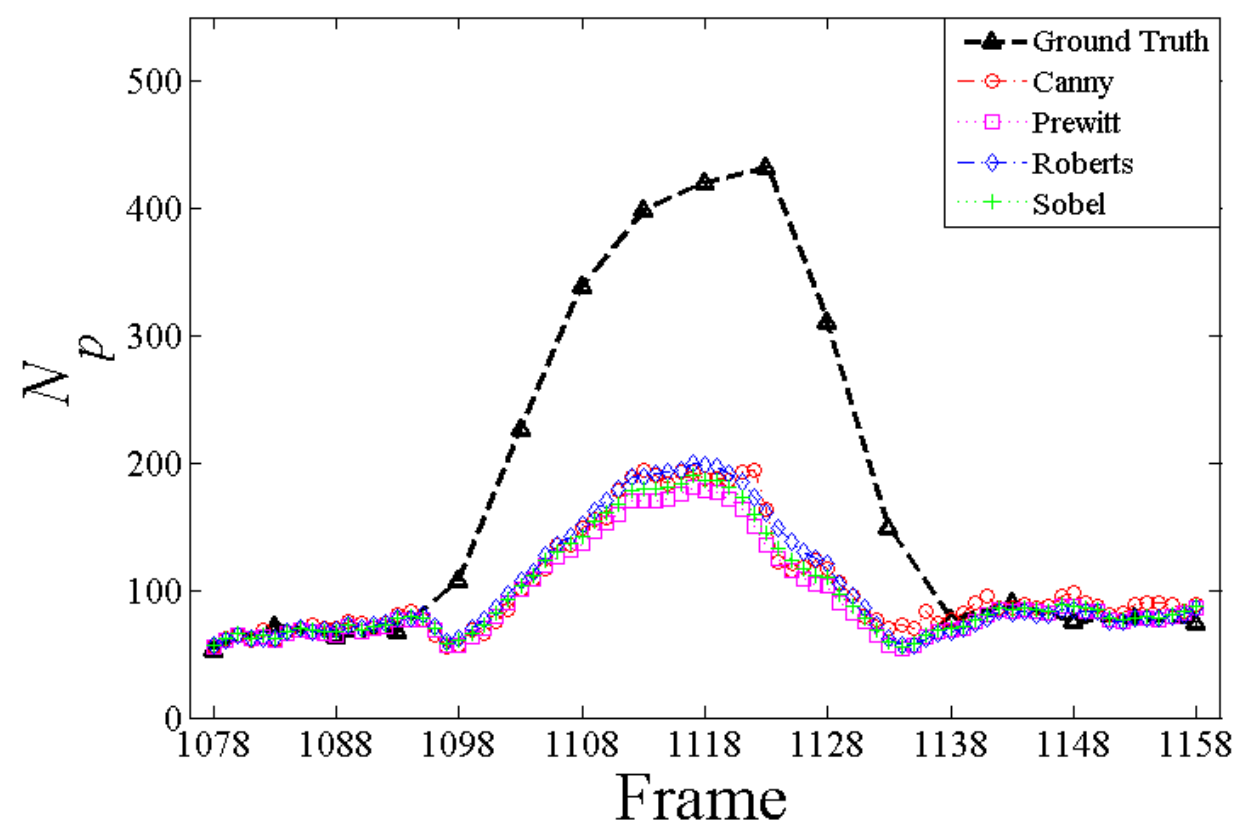

(b) Results on adjusted images

Figure 3.16: Results of estimating the particle count by the edge contours. The thresholds used varied from method to method, but each was found to minimize the MPE for that method. 


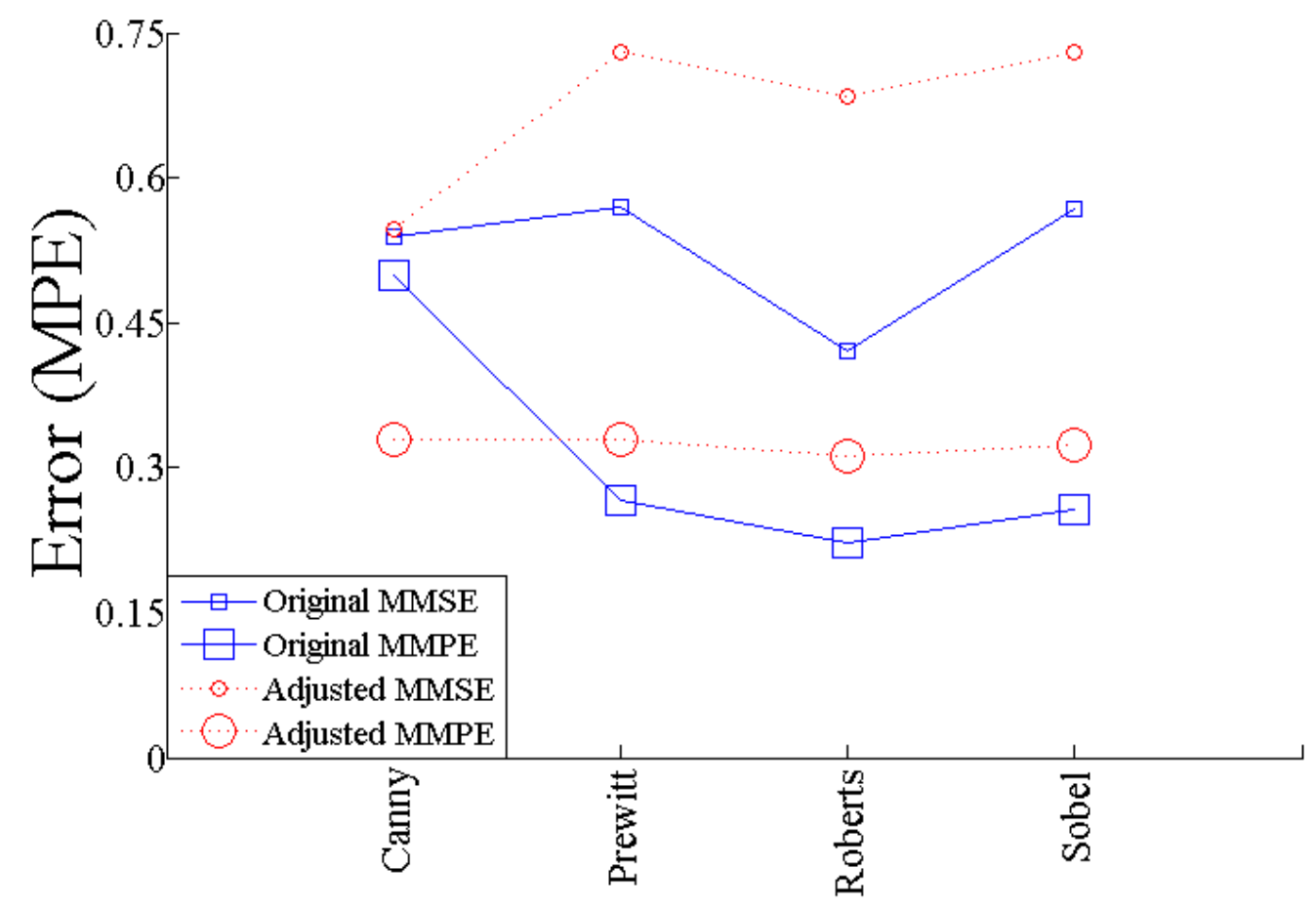

Figure 3.17: Error (MPE) for applying the minimum error thresholds for each method. Both sets of thresholds (minimum MSE and minimum MPE) were tested for each edge detection method on each image type.

Recall that the intensity images can be defined as the combination of $\mathcal{S}_{\mathcal{I}}, \mathcal{S}_{\mathcal{O}}$, and $\mathcal{S}_{\mathcal{V}}$ as in (3.11). These were the three classes expected from the output of both classifiers. The classifications were based on a number of neighborhood features extracted for each pixel. The features and classifier results are described in the following sections.

\section{Features}

To classify a single pixel $p$, eight features were collected from its neighborhood. Let $W(p)$ denote the neighborhood of pixels, or window, centered at $p$ (including $p$ ). The window size needed to be large enough to allow for proper sampling of the intensity distribution, but small enough to fit within the area of a particle. Here an $[11 \times 11]$ window was used. To reduce the bias toward features with large ranges, some of the values are standardized, i.e., placed in the range $\mathcal{R}=[0,1]$. For simplicity, the following notations are used: $|W(p)|$ represents the number of pixels in the neighborhood; $I(W(p))$ represents the intensities of 
the pixels in the neighborhood.

1. Mean $\left(f_{1}\right)$

The mean intensity of the neighborhood $W(p)$. For an image with intensities already in $\mathcal{R}=[0,1]$ this is simply

$$
\mu_{1}=\frac{1}{|W(p)|} \sum I(W(p)) .
$$

2. Standard Deviation $\left(f_{2}\right)$

The square root of the second central moment $\mu_{2}$ given by

$$
\begin{aligned}
\sigma & =\sqrt{\mu_{2}} \\
& =\sqrt{\frac{1}{|W(p)|} \sum\left(I(W(p))-\mu_{1}\right)^{2}} .
\end{aligned}
$$

3. Coefficient of Variation $\left(f_{3}\right)$

Coefficient of variation, as mentioned before, is the standard deviation as a percentage of the mean [23] and given by

$$
\mathrm{CV}=\frac{\sigma}{\mu_{1}} .
$$

4. Skewness $\left(f_{4}\right)$

The third central moment $\mu_{3}$ given by

$$
\mu_{3}=\frac{1}{|W(p)|} \sum\left(I(W(p))-\mu_{1}\right)^{3}
$$

5. Uniformity $\left(f_{5}\right)$

Measure of the intensity uniformity $u$, defined in [20] as

$$
u=\sum_{i=1}^{L} p_{i}^{2} .
$$

In [20], $u$ is described as being maximum when all graylevels are equiprobable, but in fact $u$ will be minimum for equal graylevels and maximum for a single graylevel, i.e., $p_{j}=1$ and $p_{i}=0 \forall i \neq j$. 
In the first case, when $p_{i}=\frac{1}{L} \forall i \in L$

$$
\begin{aligned}
u & =\sum_{i=1}^{L} p^{2} \\
& =\sum_{i=1}^{L}\left(\frac{1}{L}\right)^{2} \\
& =L\left(\frac{1}{L^{2}}\right) \\
u_{\min } & =\frac{1}{L} .
\end{aligned}
$$

If, in the other case, $p_{j}=1$ and all other intensities have a probability of zero, then

$$
\begin{aligned}
u & =p_{j}^{2}+\sum_{i \neq j} p_{i}^{2} \\
& =p_{j}^{2} \\
u_{\max } & =1 .
\end{aligned}
$$

This value is then in the range $\left[\frac{1}{L}, 1\right]$ which is acceptable for this experiment.

\section{Entropy $\left(f_{6}\right)$}

The entropy $H$ of an image is based on the pdf of the intensities as follows

$$
H=-\sum_{i=1}^{L} p_{i} \lg p_{i}
$$

where $\lg \equiv \log _{2} . H$ is at a maximum when all graylevels are equiprobable [58] and at a minimum if the entire image is a single, constant graylevel [31]. Letting $p_{i}=p_{j} \forall i, j$, i.e., $p=\frac{1}{L}$, the maximum entropy is

$$
\begin{aligned}
H_{\max } & =-\sum_{i=1}^{L} p \lg p \\
& =-L(p \lg p) \\
& =-L\left(\frac{1}{L} \lg \frac{1}{L}\right) \\
& =-(\lg 1-\lg L) \\
& =\lg L .
\end{aligned}
$$


Thus, for the images here with $L=256$ the maximum entropy is $H_{\max }=8$. The entropy of the intensity distribution for $W(p)$ was found and then divided by $H_{\max }$ to place the value in $\mathcal{R}$.

\section{Tenengrad $\left(f_{7}\right)$}

The Tenengrad is a focus metric based on the gradient information available in an image. The metric was used as described by Krotkov [31] where the gradient magnitudes are determined using the Sobel operators and then summed. For the local window this becomes

$$
\mathrm{TN}=\sum\|\nabla I(W(p))\|_{2}
$$

The theoretical maximum for $\mathrm{TN}$ as defined above is quite large. Instead of using this value however, the maximum from the training data is used to keep the values of TN in the perspective of the particle images. For the training data with intensities in $\mathcal{R}$, $\mathrm{TN}_{\max }=8.1992$.

The set of features $\left\{f_{i}\right\}_{i=1}^{7}$ extracted from $W(p)$ form the feature vector $F(p)=$ $\left[f_{1}(W(p)) f_{2}(W(p)), \ldots, f_{7}(W(p))\right]$. Each feature vector is then classified either by existing training samples $(k-\mathrm{NN})$ or by its relation to other feature vectors in the image ( $k$-means).

\section{k-Nearest Neighbor}

The one-nearest neighbor (1-NN) classifier is the simplest of a family of $k$-nearest neighbor classifiers. Each operates by measuring the distance (typically Euclidean) between the point of interest and a set of training points whose classes are known [29]. The most prevalent class from the set of the $k$ closest training points is given to the point of interest. Thus, the 1-NN simply classifies a point based on the nearest point in the set of training samples.

The training data was gathered manually by identifying $[11 \times 11]$ neighborhoods on in-focus particles, out-of-focus particles, and areas where the background was empty. To ensure a representative sample was collected, 50 neighborhoods of each class were identified. Using the leave-one-out cross-validation method, the classification error was checked based on the training points. For $n$ points, this method uses $(n-1)$ samples to classify the unselected point and repeats $n$ times [29]. The error estimates based on this technique for 
values of $k \in\{2 j-1\}_{j=1}^{5}$ are shown in Figure 3.18. From these results, $k=3$ was chosen for classifying other particle images. Error values for $k=3$ and $k=9$ were both $8 \%$.

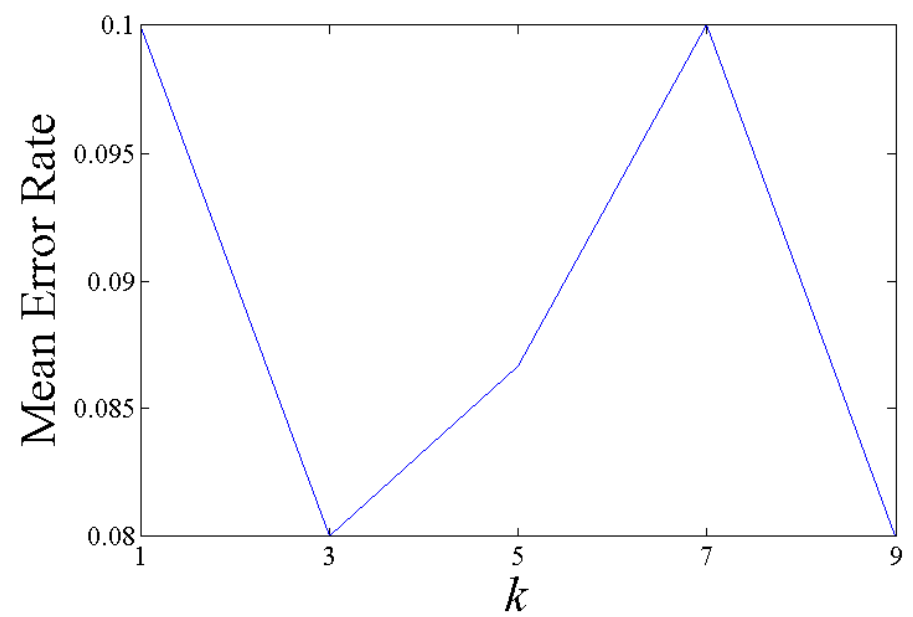

Figure 3.18: Error estimates for $k$-NN classifier based on training data.

The result of applying the classification technique on an example image is shown in Figure 3.19. A number of particles were jointly classified as in-focus and out-of-focus, similar to the intensity thresholding techniques discussed earlier.

The 3-NN classifier was applied to the set of images $\mathcal{I}$ as before using four different sets of features and the results are shown in Figure 3.20. "All Features" is the entire set of 7 features, "FSS" is a set of features found by exhaustive feature subset search meant to give the best classification results on the training data which was $\left\{f_{1}, f_{3}, f_{4}, f_{7}\right\}$, "Moments" is the set of central moments $\left\{f_{1}, f_{2}, f_{4}\right\}$, and "Mean" is simply the mean intensity $f_{1}$. Notice there was little difference between using all 7 features and using only the subset of 4. Given the results of the intensity-based methods, it is not surprising that the mean alone performed decently.

\section{$k$-Means Clustering with Decision Rule}

Many clustering techniques attempt to partition the patterns into groups whose classes are unknown. The $k$-means algorithm is a simple, efficient, and effective partitional clustering algorithm that works by minimizing the distance (typically Euclidean) between the features and a set of $k$ centroids [29]. These class centers can often be initialized by the user or determined at random, but must be iteratively updated to find the optimal 


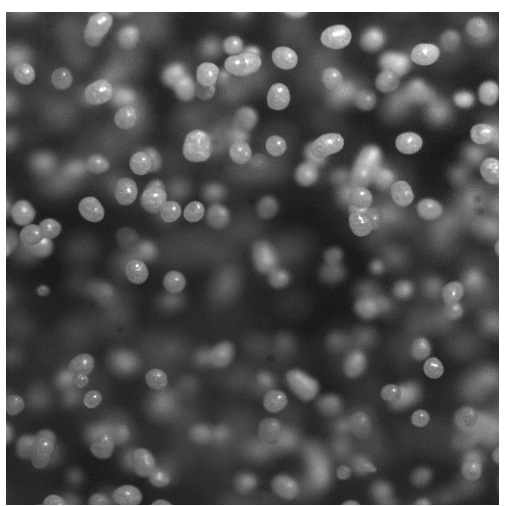

(a) Original Image

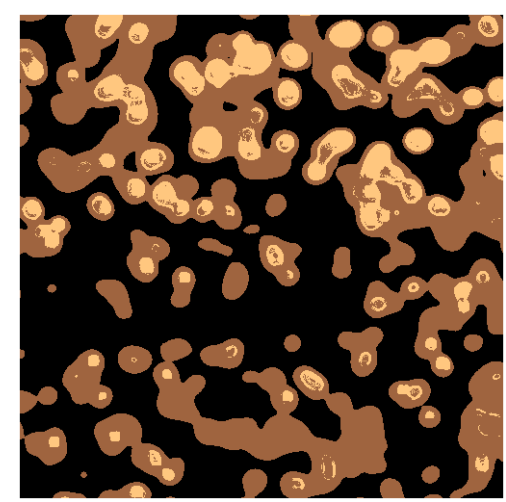

(b) KNN Result

Figure 3.19: Example of $k$-NN classification. The left image is the original and the right is the result of the $k$-NN classification. The light, medium, and dark regions are $\mathcal{S}_{\mathcal{I}}, \mathcal{S}_{\mathcal{O}}$, and $\mathcal{S}_{\mathcal{V}}$, respectively.

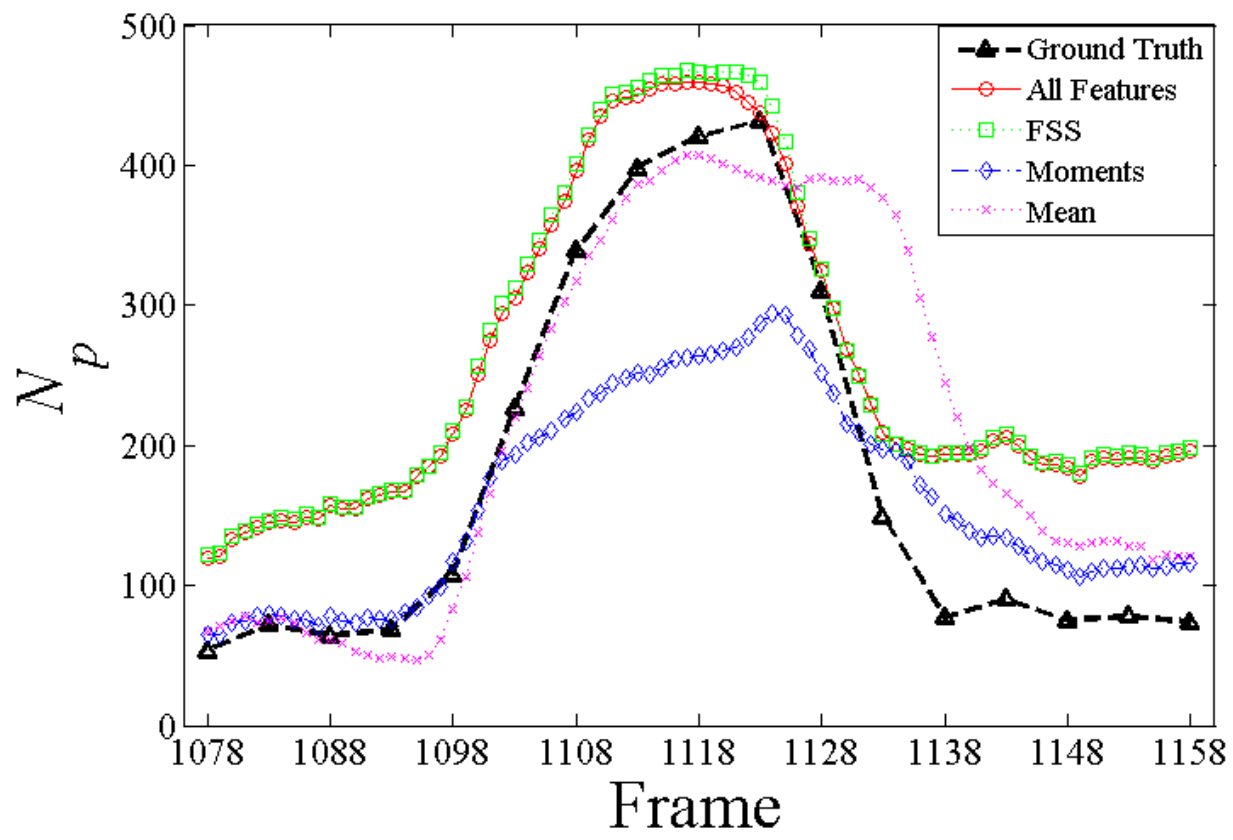

Figure 3.20: Results of $k$-NN classification on the set of test images $\mathcal{I}$. Only the training data was collected on the original images, so only they were tested. 
locations. In each iteration, every feature point is classified by the nearest centroid. The position of each centroid is then updated based on the new pattern classifications. This will continue until the within-cluster variations about their centroid is minimized [29]. As before, three classes are expected from these images, so this algorithm was used with $k=3$.

Once the clusters were found, they had to be classified as either $\mathcal{S}_{\mathcal{I}}, \mathcal{S}_{\mathcal{O}}$, or $\mathcal{S}_{\mathcal{V}}$. Generally, the in-focus particles had the highest intensities and the background was mostly black, having the lowest intensities. A decision rule was then created to classify the cluster with the highest mean intensity as $\mathcal{S}_{\mathcal{I}}$ and the lowest as $\mathcal{S}_{\mathcal{V}}$. The remaining cluster was then classified as $\mathcal{S}_{\mathcal{O}}$.

The clustering algorithm was applied to the training set used by the $k$-NN classifier. The four feature sets described earlier were used in for clustering and each gave more than a $20 \%$ error, which was larger than that of $k$-NN. The failure was primarily in classifying the in-focus particles while there was zero error in classifying the background. Given the importance of the in-focus particles and its inability to find them, the $k$-means clustering method was not tested on the set of images.

\subsection{Direct Identification by Specular Highlights}

Another potential method for directly counting particles was to use the specular highlights from the particle surfaces. While occasionally found on particles slightly removed from the focal plane, these features typically indicated the presence of an in-focus particle. Accurate detection of these highlights could serve as a rich source of information.

Previously, the high-intensity noise $I^{s}$ was found from the specular highlight suppression (SHS) algorithm described in $\S 2.3$. This was again used but with an emphasis on identifying individual reflections. To begin, the noise image $I^{s}$ was thresholded to eliminate weak changes in the intensity due to the median filter from the SHS algorithm. To do so, yet retain all of the highlights, this threshold was set fairly low at $T_{s}=0.1$.

After thresholding the image, a median filter was applied to the result to further reduce noise in the specular regions. The image prior to filtering was binary. By applying the filter with an odd sized window, it was assured that the result would also be binary. All contiguous regions of marked pixels were found and the centroid determined. These centroids served as particle markers. Results of this algorithm for window sizes [3 $\times 3]$, $[5 \times 5]$, and $[7 \times 7]$ on both image types are shown in Figure 3.21. 


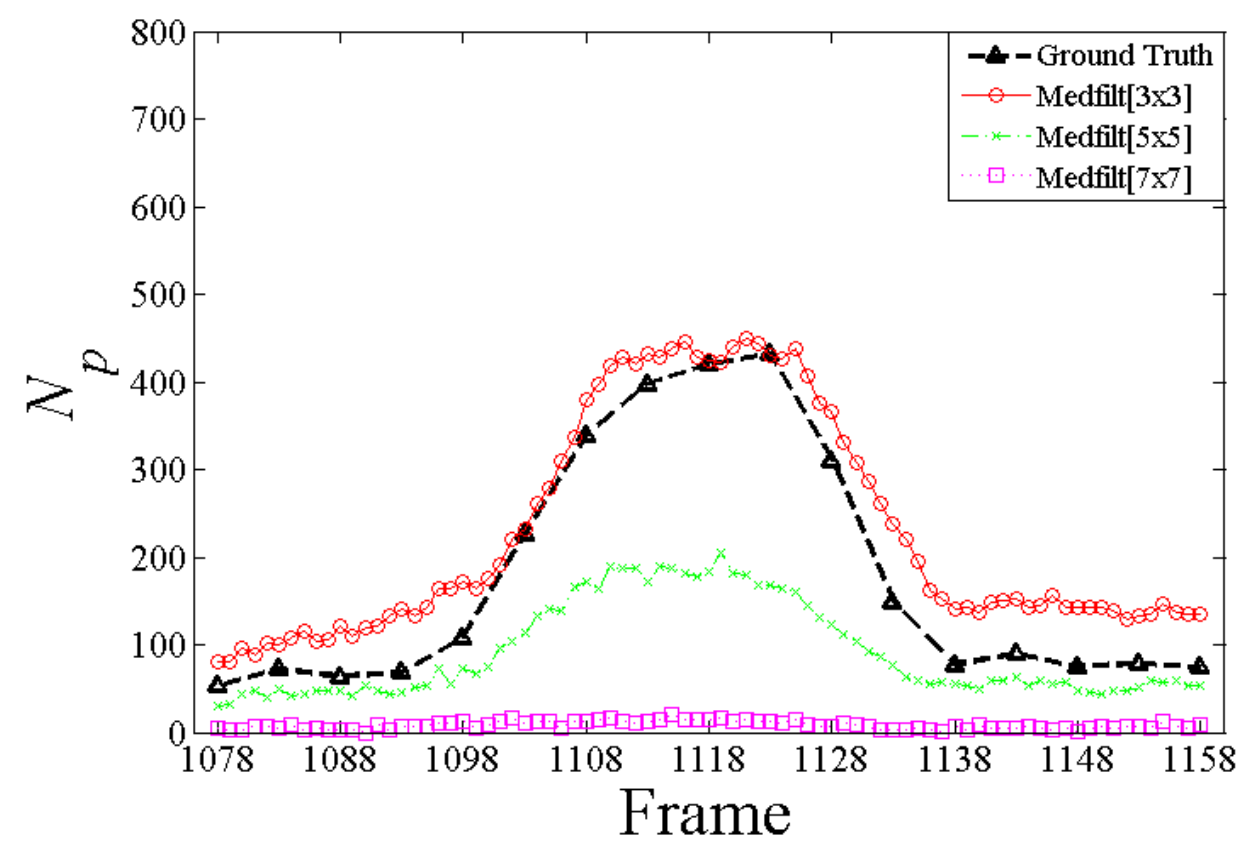

(a) Results on original images

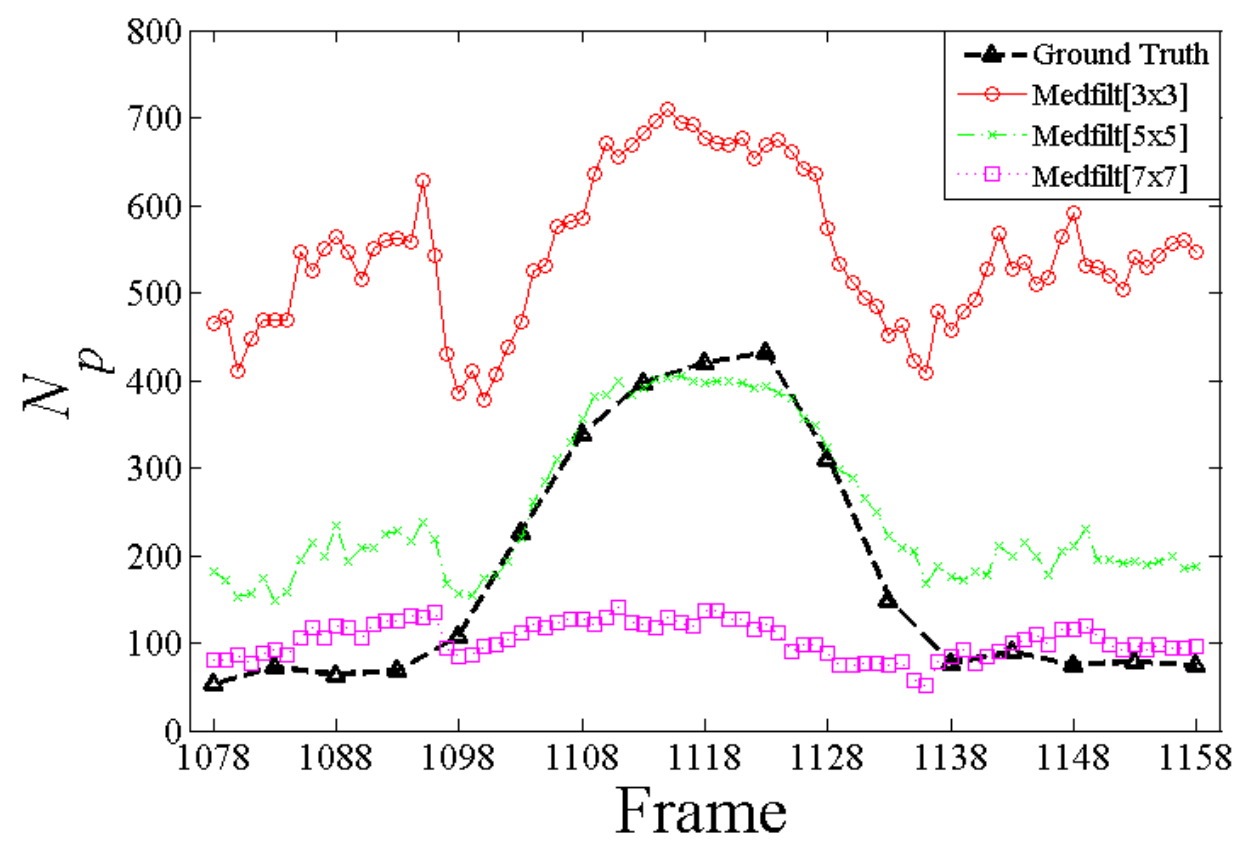

(b) Results on adjusted images

Figure 3.21: Results of estimating the particle count by the number of specular highlights. No account has been made of false positives. 
It was noticed that some false positives were detected along particle boundaries, especially when particles were secluded so that their surface was well contrasted by the dark background. To take these into account the edge map of the output of the SHS algorithm was found using the Canny edge detector. The locations of candidate specularities were then compared to the edge map and any found lying on a contour were thrown out as false positives. To account for rounding issues as well, the edge map was first dilated with a kernel of ones to thicken the contours. Results after eliminating candidate specularities in this fashion are shown in Figures 3.22 and 3.23 for kernels of size $[2 \times 2]$ and [3 $\times 3]$, respectively.

The error of each technique was assessed against the ground truth data for both types of images and shown in Figure 3.24. The label $M_{0}$ is the initial method were no account was made for false positives, $M_{1}$ denotes the $[2 \times 2]$ dilation kernel, and $M_{2}$ the $[3 \times 3]$ kernel. The error was at a minimum for the $[3 \times 3]$ dilation kernel for the original and adjusted images.

\subsection{Estimated Solids Concentration}

Once the total particle area $\mathcal{A}$ or the estimated particle count $\widehat{N}_{p}$ has been determined, a 2-dimensional solids fraction can be estimated. Using the total area $\mathcal{A}$, the solids area fraction $\psi_{p}$ can be estimated by

$$
\begin{aligned}
\widehat{\psi}_{p} & =\frac{\mathcal{A}}{|\mathcal{X}||\mathcal{Y}|} \\
& =\frac{\left|\mathcal{S}_{\mathcal{I}}\right|}{|\mathcal{X}||\mathcal{Y}|}
\end{aligned}
$$

where $|\mathcal{X}|$ and $|\mathcal{Y}|$ denote the number of rows and columns in the image, respectively, and $\left|\mathcal{S}_{\mathcal{I}}\right|$ is the number of pixels classified as in-focus. Using the estimated particle count relies on the mean particle diameter $d_{p}$ to first determine the area $\mathcal{A}$ from (3.6), but then $\psi$ can be determined directly from (3.67).

The void fraction, or voidage, is a 3-dimensional measurement of the empty space dependent on the particle volume. This measure is often denoted as simply $\varepsilon$, although here it will be explicitly labeled $\varepsilon_{v}$. The void fraction and the solids (particle) fraction $\varepsilon_{p}$ are complementary values, i.e., $\varepsilon_{v}+\varepsilon_{p}=1$. In order to determine the void fraction $\varepsilon_{v}$ for use in modeling, the 2-dimensional area $\psi_{p}$ must be transformed into a 3 -dimensional volume 


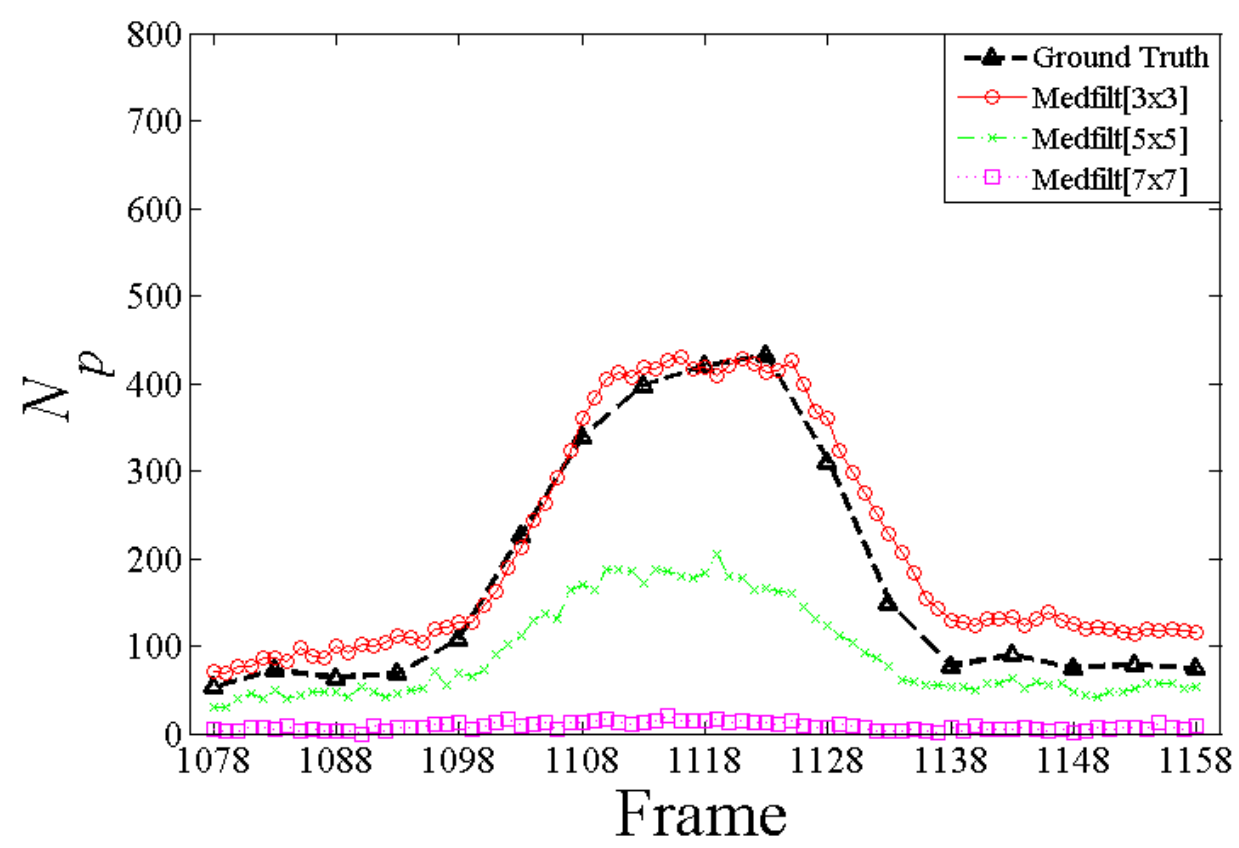

(a) Results on original images

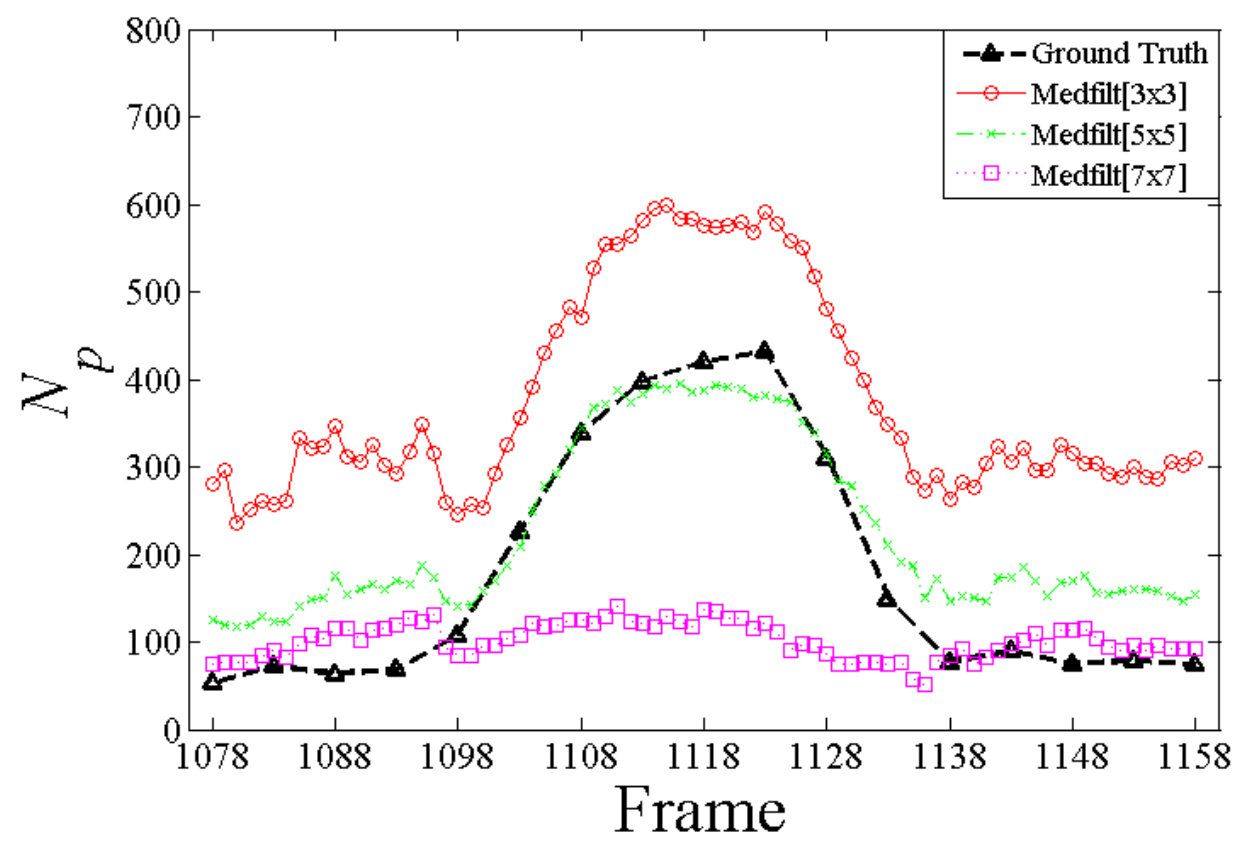

(b) Results on adjusted images

Figure 3.22: Results of estimating the particle count by the number of specular highlights. Edges detected using Canny filter on the $I-I^{s}$ image were dilated by a [2×2] kernel of ones. 


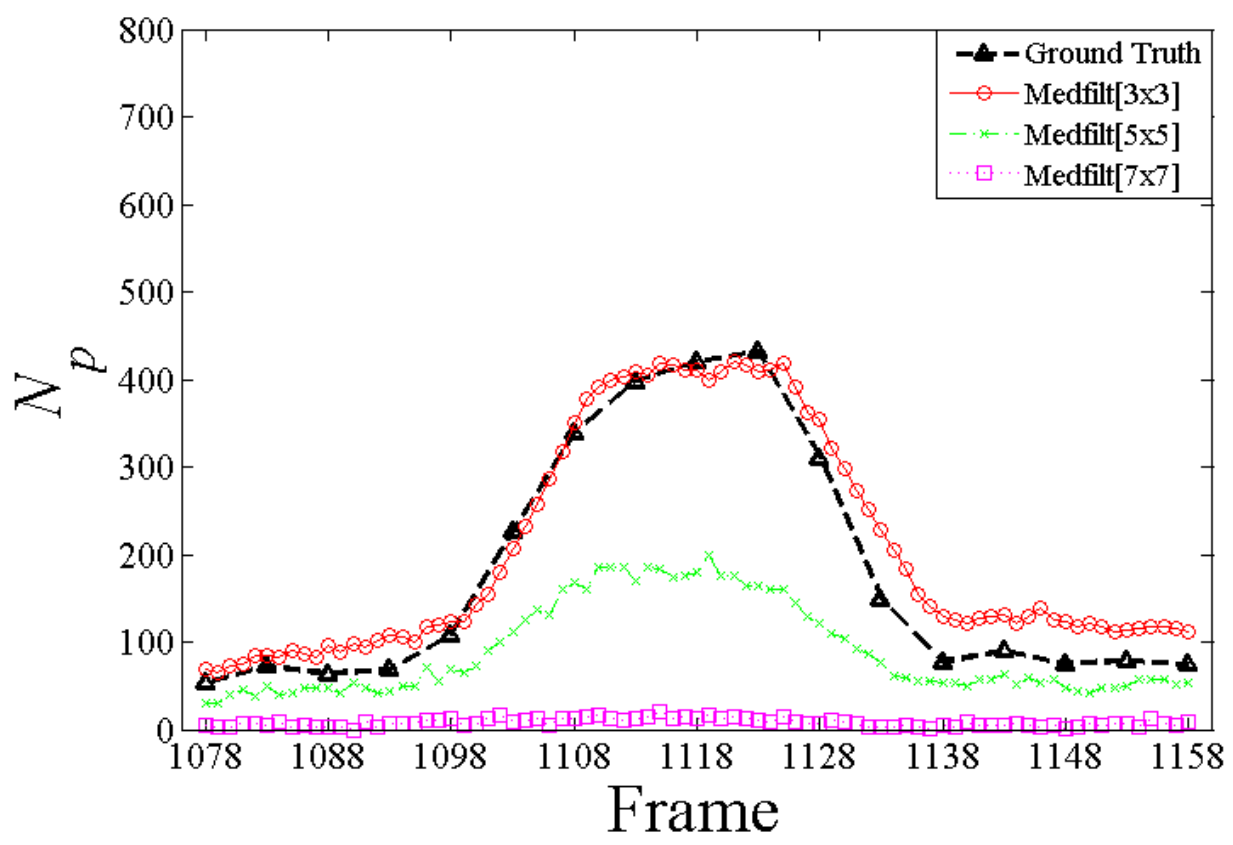

(a) Results on original images

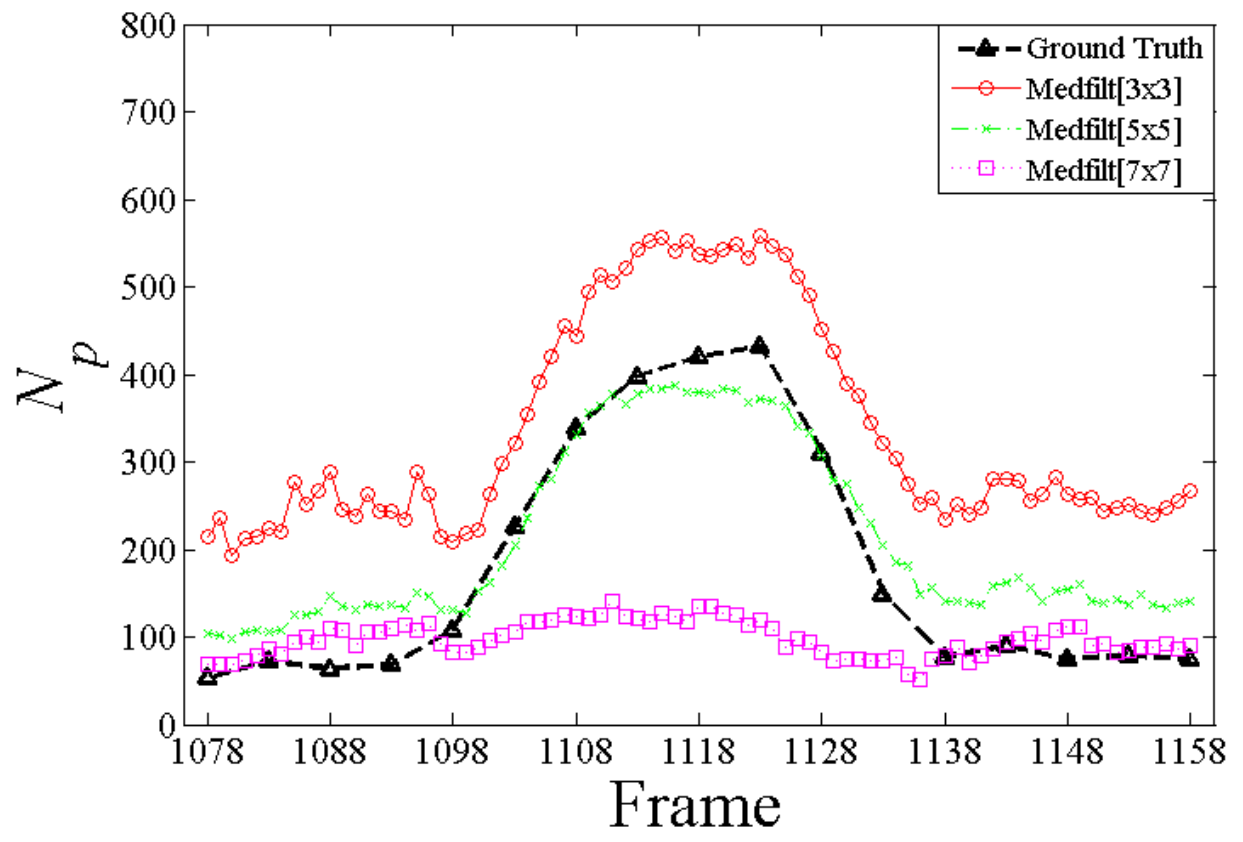

(b) Results on adjusted images

Figure 3.23: Results of estimating the particle count by the number of specular highlights. Edges detected using Canny filter on the $I-I^{s}$ image were dilated by a [3 $\left.\times 3\right]$ kernel of ones. 


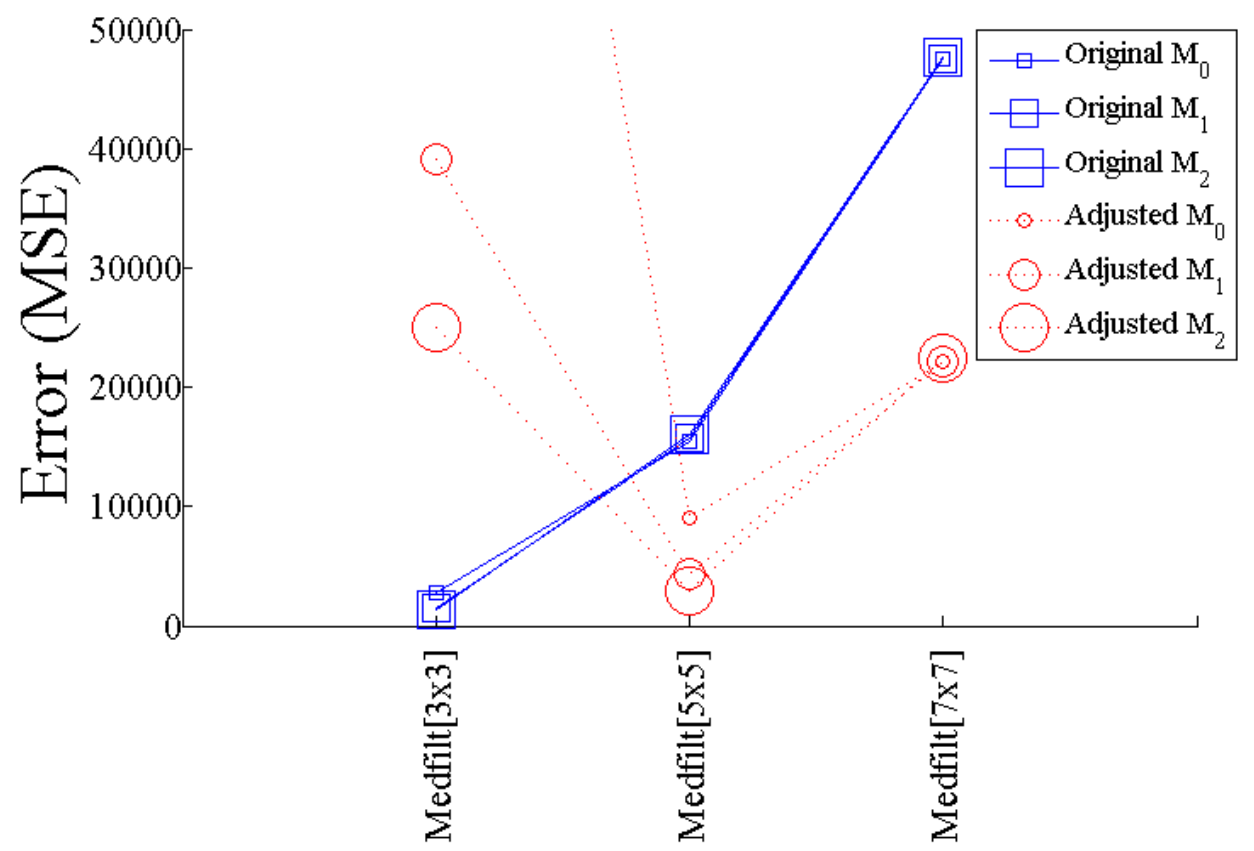

(a) MSE

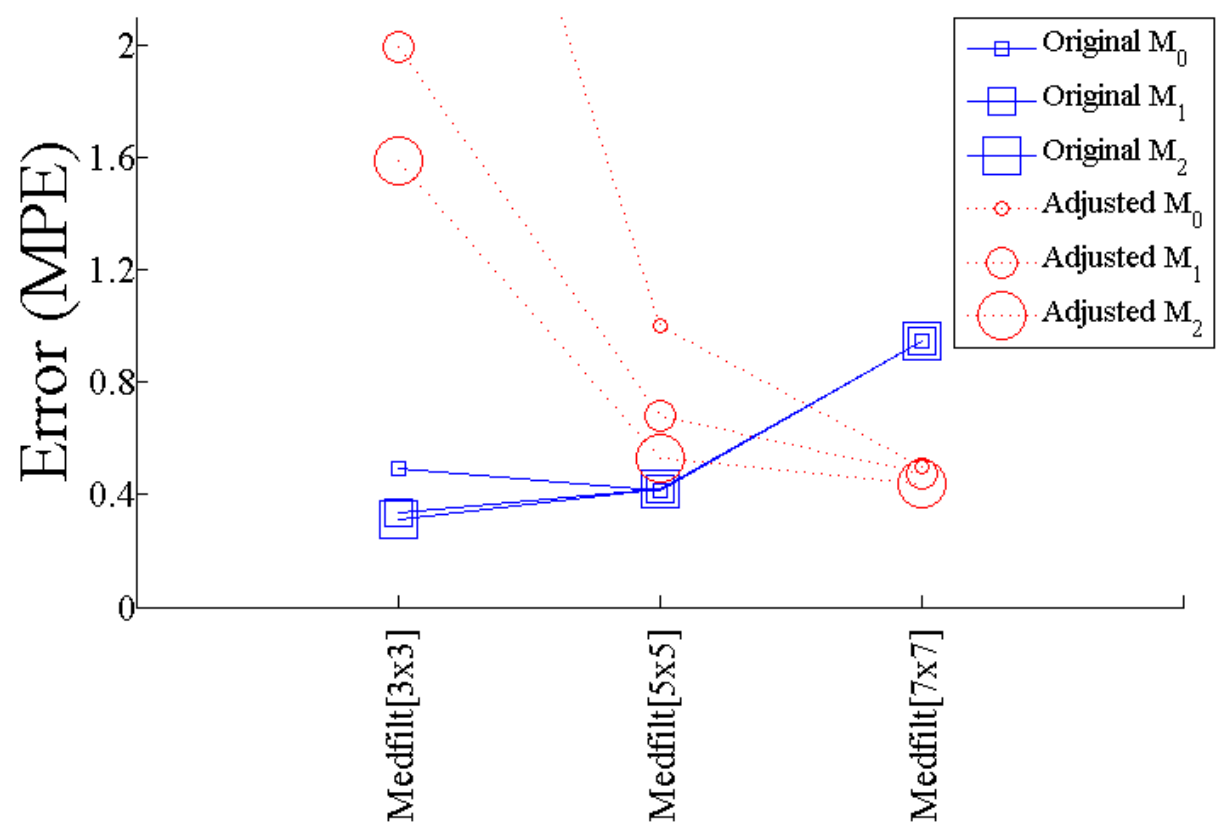

(b) MPE

Figure 3.24: Error values for estimating the particle count by the number of detected specular highlights. The clipped error values were both for "Adjusted $M_{0}$ " with $E_{\mathrm{MSE}} \approx$ 143000 and $E_{\mathrm{MPE}} \approx 3.9$. 
$\varepsilon_{p}$

Various methods have been suggested for converting a void fraction from 2 to 3 dimensions, many of which arise from studies of 2D flow simulations [37]. The most basic method is to assume the particle volume is a single layer thick, i.e., the particle depth is $d_{p}$. It was suggested that this is a poor assumption, citing that the estimated voidage is generally too high [24]. Two very similar equations have been proposed that are based on the relationship between a hexagonal lattice of spheres in 2 and 3 dimensions, assuming inter-particle distances are equal [25, 46].

The transformation derived by Hoomans et al. [25] is given by

$$
\varepsilon_{v}^{\mathrm{H}}=1-\frac{2}{\sqrt{\pi \sqrt{3}}} \psi_{p}^{\frac{3}{2}} .
$$

From Ouyang and $\mathrm{Li}[46]$ the transformation is

$$
\varepsilon_{v}^{\mathrm{O}}=1-\frac{\sqrt{2}}{\sqrt{\pi \sqrt{3}}} \psi_{p}^{\frac{3}{2}}
$$

Notice there is very little difference between the two, although Hoomans argues that (3.70) gives estimates that are too high [24]. Li, a contemporary of Hoomans, explained that the depth assumed by (3.69) is $2 \times 3^{-0.75} d_{p}$ or $0.8774 d_{p}$ [37].

The void fraction $\varepsilon_{v}$ determined for the baseline data using (3.69) is shown in Figure 3.25. For the typical dilute phase ( $N_{p} \leq 90$, the median value), the void fraction is, on average, approximately $96 \%$. For the maximum particle density, the voidage dropped to approximately $45 \%$.

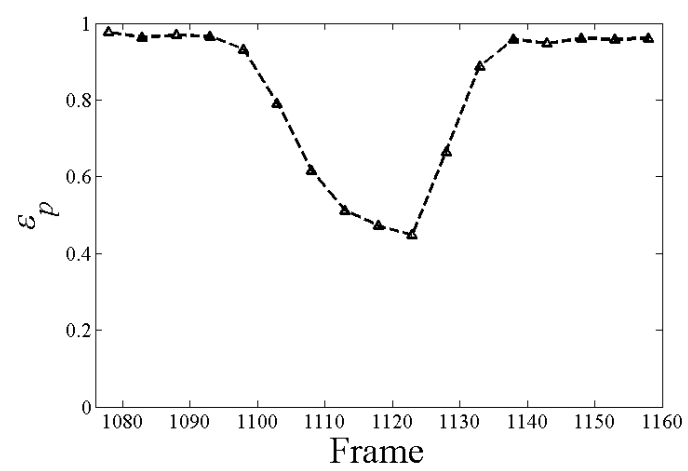

Figure 3.25: The ground truth (baseline) data converted to a void fraction in 3 dimensions. 


\subsection{Summary}

A number of techniques were evaluated based on their ability to estimate the number of particles present in a set of images. By analyzing the MPE error alone it was determined that the Roberts edge filter produced the best results. The method of counting specularities, however, has the added benefit of detecting individual particles. Any of the classification methods evaluated would need to be combined with another technique to determine the locations of individual particles. 
This page is intentionally blank. 


\section{Chapter 4}

\section{Particle Tracking}

\subsection{Optical Flow}

Image motion is the $2 \mathrm{D}$ projection of $3 \mathrm{D}$ object motion on the image plane [4]. The approximation of this 2D motion field is typically referred to as optical flow [3]. The basic assumption in this measurement is that the brightness or intensity of objects remains (approximately) constant while undergoing motion [28, 4]. Considering the image plane as a surface, denote the brightness of each point $(x, y)$ at time $t$ as $I(x, y, t)$. The assumption can then be written as

$$
I(x, y, t)=I(x+\Delta x, y+\Delta y, t+\Delta t) .
$$

A Taylor series expansion of the right-hand side gives

$$
I(x, y, t)=I(x, y, t)+\Delta x \frac{\partial I}{\partial x}+\Delta y \frac{\partial I}{\partial y}+\Delta t \frac{\partial I}{\partial t}+O^{2}
$$

where $O^{2}$ contains the second and higher order terms. Assuming the terms in $O^{2}$ are negligible, rearranging (4.2) gives

$$
\frac{\Delta x}{\Delta t} \frac{\partial I}{\partial x}+\frac{\Delta y}{\Delta t} \frac{\partial I}{\partial y}+\frac{\partial I}{\partial t}=0
$$

Let $I_{x}$ and $I_{y}$ be the spatial gradients, $I_{t}$ the temporal gradient ${ }^{1}$, and $u$ and $v$ the

\footnotetext{
${ }^{1}$ The temporal derivative is commonly approximated by finite backward differences, utilizing just two frames [4].
} 
components of the image velocity in the $x$ and $y$ directions, respectively. This simplifies the previous equation to

$$
\begin{aligned}
I_{x}(x, y, t) u+I_{y}(x, y, t) v+I_{t}(x, y, t) & =0, \\
\nabla I(x, y, t) \cdot \mathbf{v}+I_{t}(x, y, t) & =0
\end{aligned}
$$

where $\mathbf{v}=[u v]^{T}$ and $\nabla$ is the gradient operator. This is known as the optical flow constraint equation and is linear with respect to the unknowns $u$ and $v$ [28].

In order to determine the two unknowns $u$ and $v$, additional constraints are required [3]. The classical Horn and Schunck model [28] uses a global smoothness constraint on the velocity, citing the difficulty in recovering the entire velocity field if each point on the surface can move independently. This is an inadequate constraint for the images in this study given the random motion of the particles. Also, computing the optical flow for the entire surface would be of considerable computational cost. A more applicable constraint is a model for locally constant motion within small neighborhoods, i.e., smaller than a particle. This can be implemented by separating each image into a number of equal-sized blocks which are analyzed independently or driven by specific features in the image but either will result in a velocity field with a lower density than the global model and likely a lower error [3].

\subsubsection{Lucas-Kanade Feature Tracker}

One method for evaluating the local optical flow is to solve a weighted least-squares of the linear constraint (4.5) while holding the displacements $u$ and $v$ constant [3]. The technique, first introduced by Lucas and Kanade [38, 39], minimizes the energy functional

$$
E=\sum_{(x, y) \in \Omega} W^{2}(x, y)\left(\nabla I(x, y, t) \cdot \mathbf{v}+I_{t}(x, y, t)\right)^{2}
$$

where $\Omega$ is the spatial neighborhood and $W(x, y)$ is a window function that weights the pixels in the neighborhood, giving more importance to those near the center [3]. 
Differentiating the functional with respect to the two components of $\mathbf{v}$ gives

$$
\begin{aligned}
& \frac{\partial E}{\partial u}=\sum_{(x, y) \in \Omega} 2 W^{2}\left(\nabla I(x, y, t) \cdot \mathbf{v}+I_{t}(x, y, t)\right) I_{x}(x, y, t), \\
& \frac{\partial E}{\partial v}=\sum_{(x, y) \in \Omega} 2 W^{2}\left(\nabla I(x, y, t) \cdot \mathbf{v}+I_{t}(x, y, t)\right) I_{y}(x, y, t)
\end{aligned}
$$

where the energy will be minimized when $\nabla E=0$. Setting each to zero and rearranging gives

$$
\begin{aligned}
\sum_{\Omega} W^{2}\left(I_{x}^{2} u+I_{x} I_{y} v\right)+\sum_{\Omega} W^{2} I_{x} I_{t} & =0, \\
\sum_{\Omega} W^{2}\left(I_{x} I_{y} u+I_{y}^{2} v\right)+\sum_{\Omega} W^{2} I_{y} I_{t} & =0, \\
{\left[\begin{array}{cc}
\sum W^{2} I_{x}^{2} & \sum W^{2} I_{x} I_{y} \\
\sum W^{2} I_{x} I_{y} & \sum W^{2} I_{y}^{2}
\end{array}\right]\left[\begin{array}{l}
u \\
v
\end{array}\right]+\left[\begin{array}{c}
\sum W^{2} I_{x} \\
\sum W^{2} I_{y}
\end{array}\right] I_{t} } & =0 .
\end{aligned}
$$

The solution of the least-squares minimization over the neighborhood $\Omega$ is then

$$
\mathbf{v}=A^{-1} b
$$

where $\mathbf{v}=[u v]^{T}$ and

$$
\begin{aligned}
A & =\left[\begin{array}{cc}
\sum W^{2} I_{x}^{2} & \sum W^{2} I_{x} I_{y} \\
\sum W^{2} I_{x} I_{y} & \sum W^{2} I_{y}^{2}
\end{array}\right], \\
b & =-\left[\begin{array}{c}
\sum W^{2} I_{x} \\
\sum W^{2} I_{y}
\end{array}\right] I_{t} .
\end{aligned}
$$

\subsubsection{Image Pyramid Implementation}

Hierarchical, coarse-to-fine methods can be used to detect a larger range of possible displacements without severely affecting the computational efficiency [4]. An image pyramid (Figure 4.1) is constructed such that the base level $L_{0}$ is the original, high-resolution image and each level $L_{k+1}$ is a filtered and subsampled version of the image from level $L_{k}$ for $k \geq 0$ [1]. For optical flow, initial estimates of the displacements are formulated on the coarsest level and refined on each of the subsequent pyramid levels, each with a higher 
resolution image.

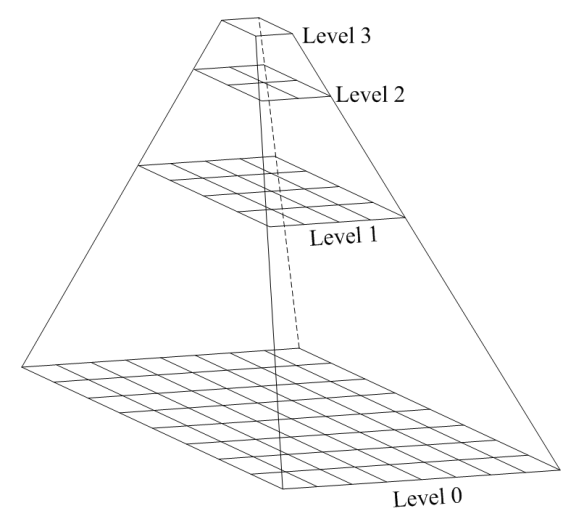

Figure 4.1: Basic construction of an image pyramid. Level 0 represents the original, highresolution image; each subsequent step up is a filtered and subsampled version.

A hierarchical implementation of the Lucas-Kanade optical flow was originally suggested in the seminal work of Lucas and Kanade [38, 39]. Restricting the computation of optical flow to useful image features and a method to identify these was later described by Tomasi and Kanade [59]. A Lucas-Kanade feature tracker was described in great detail by Bouguet [6] without the inclusion of feature identification. The feature tracker described in [6] was implemented here to track particle features determined both manually and automatically.

One might be tempted to use the particle boundaries as features for tracking, however, as was seen in the previous chapter, as the concentration of particles increases, the amount of information along the particle boundaries decreases. Specifically, boundary information is lost when particles are in physical contact or appear to overlap with another particle moving in the background. An example of applying the Lucas-Kanade feature tracker to a particle boundary is shown in Figure 4.2. The boundary points in Figure 4.2(a), shown as bright lines, were supplied manually; those seen in Figure 4.2(b) are the positions estimated by the Lucas-Kanade feature tracker. Many of the boundary points were incorrectly tracked due to the out-of-focus particle moving in the background. Occasionally an entire particle boundary was correctly tracked across a few frames, but this was limited to very dilute concentrations with very few background particles. 


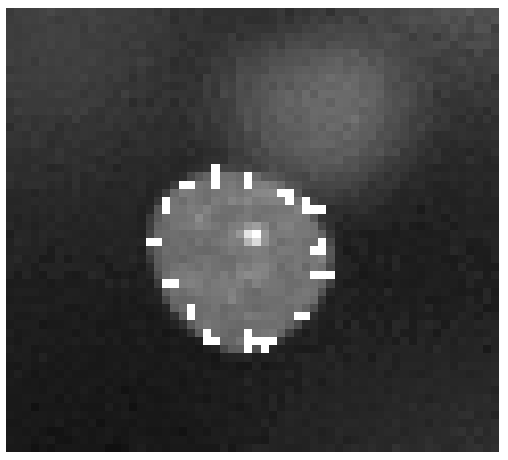

(a) Frame $I_{8}$

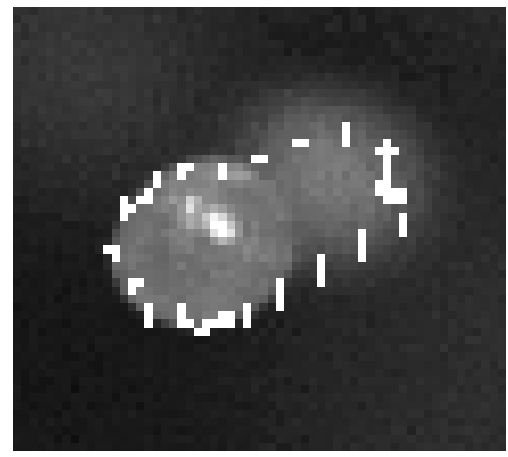

(b) Frame $I_{9}$

Figure 4.2: Example of tracking the boundary of a single particle. The boundary was tracked from from $I_{8}$ to $I_{9}$ using the Lucas-Kanade feature tracker.

\subsection{Tracking Specular Highlights}

A source of information found in this study to be surprisingly reliable is the specular reflections visible on the surface of the particles. Tracking these highlights is made easier by the absence of occluding particles in the images. The highlights were seen to consistently appear for particles on the focal plane and an automatic method for detecting these highlights has already been defined.

Given two successive images $I_{k}$ and $I_{k+1}$, the positions of the specular highlights were first determined from the method described in $\S 3.4$ for each image, giving sets of coordinates $S_{k}=\left\{\left[x_{i}^{k} y_{i}^{k}\right]^{T}\right\}_{i=1}^{n_{k}}=\left\{\mathbf{s}_{i}^{k}\right\}_{i=1}^{n_{k}}$ and $S_{k+1}=\left\{\mathbf{s}_{i}^{k+1}\right\}_{i=1}^{n_{k+1}}$ for $I_{k}$ and $I_{k+1}$, respectively. From the process of finding the highlights, the high-intensity noise images $I_{k}^{s}$ and $I_{k+1}^{s}$ were retained from (2.20). The points in $S_{k}$ were then tracked to $I_{k+1}$ using the noise images. These noise images $I^{s}$ predominantly correspond to the specular highlights, as seen in Figure 4.3. Utilizing the high-intensity noise alone significantly reduces the number of features in the image and enables to the tracker to operate more effectively.

While much simpler than the original images, the hierarchical structure of the tracking algorithm can create difficulties in correctly tracking highlights in the higher levels of the image pyramid. While the tracking window has been constrained to be smaller than a particle, in coarse levels of the pyramid, multiple highlights can be present within a single window. This allows multiple motions to exist within the local neighborhood which is poorly handled by the local model being employed [4].

To counter this, a simple algorithm was developed to eliminate the improperly 


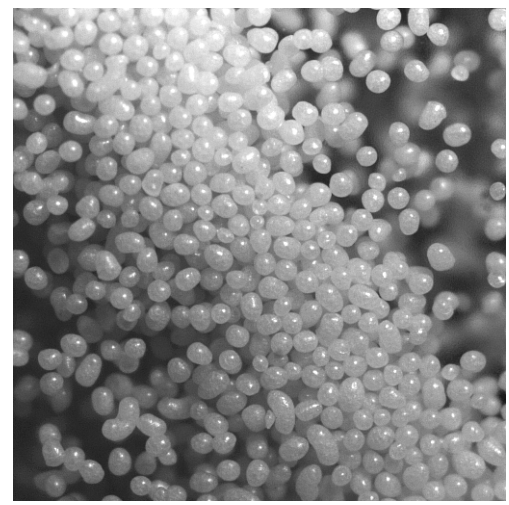

(a) Original: $I(x, y)$

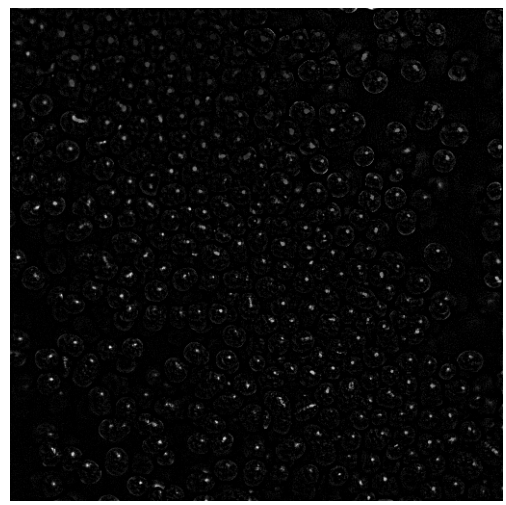

(b) Noise: $I^{s}(x, y)$

Figure 4.3: An original image and its associated high-intensity noise as determined by the SHS algorithm described in $\S 2.3$. The noise image $I^{s}$ has been transformed using the contrast stretch operator for clarity.

tracked highlights. The estimated positions in the succeeding frame $\widehat{S}_{k+1}$, as determined by the tracking algorithm, are compared to the known positions of highlights $S_{k+1}$. The algorithm matches all estimated and true positions, starting with the closest, whose distance from each other remains within a threshold $T_{d}$. The threshold was kept small $\left(T_{d}=4\right)$ to limit the number of false matches. The algorithm only requires a set of known positions and a set of estimated positions and is detailed in Algorithm 1. The output of the algorithm is a vector containing the indices of the matched points. Any estimated positions $\hat{\mathbf{s}}_{r}^{k+1} \in \widehat{S}_{k+1}$ where $\operatorname{Match}[r]=0$ is unmatched and any positions $\mathbf{s}_{r}^{k+1} \in S_{k+1}$ such that $r$ is not found within Match is considered a new particle.

An example of applying the feature tracker to a specular highlight is shown in Figure 4.4 where the same particle from Figure 4.2 has been tracked. In the figure, the large squares mark the position of the specular highlight in the current frame, while the circle denotes its past position. Unlike when tracking the boundary, the background particle did not affect the ability of the tracker to find the correct particle.

\subsection{Results}

The Lucas-Kanade feature tracker has been applied to the set of images $\mathcal{I}=$ $\left\{I_{1078+5 i}\right\}_{i=0}^{16}$, described in $\S 3.2$, to evaluate its effectiveness. For each image in $\mathcal{I}$, the specular highlights were found and tracked to the succeeding image in the sequence of 


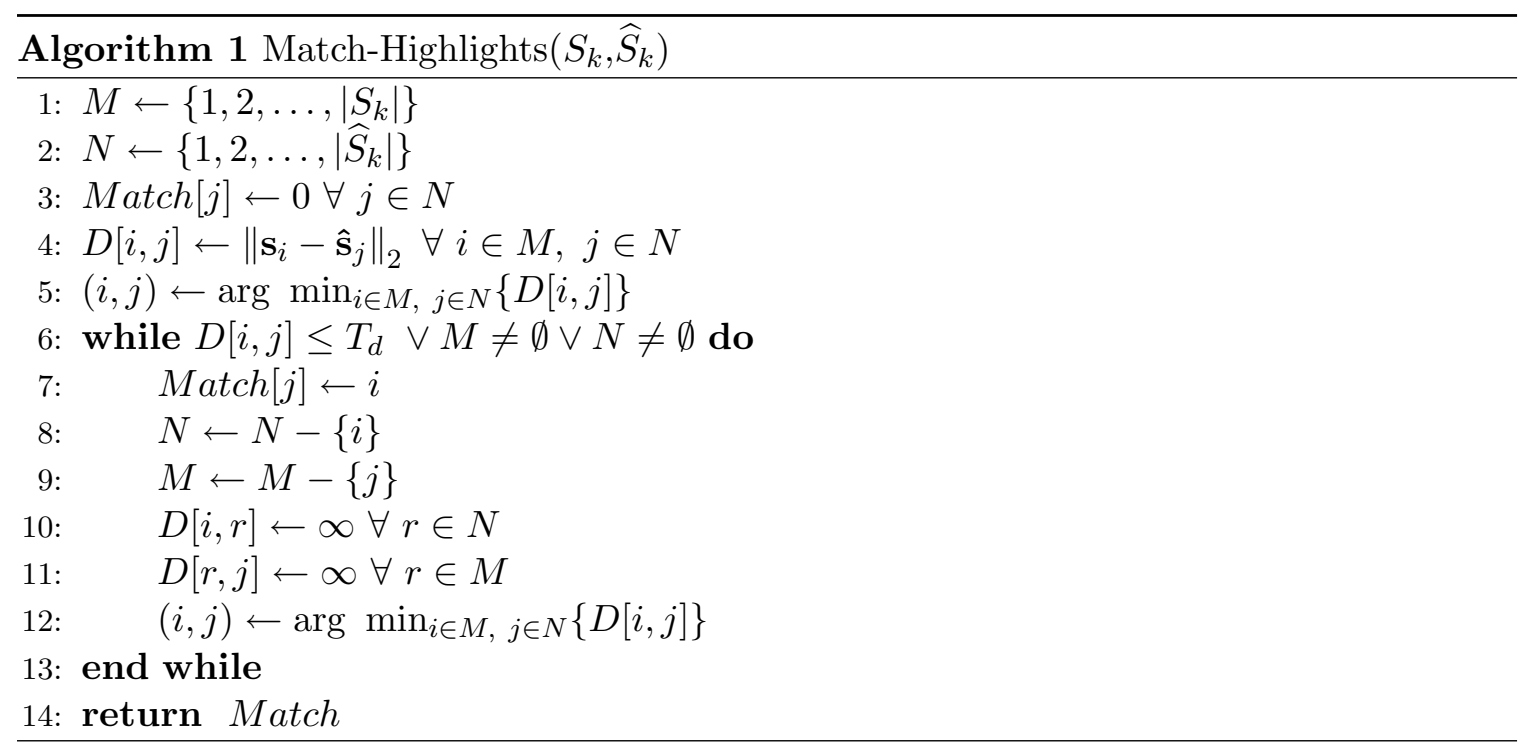

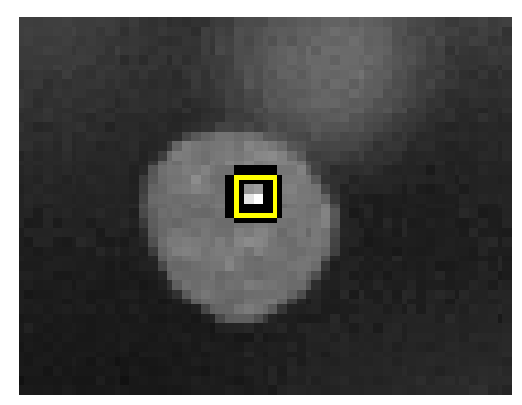

(a) $I_{8}$

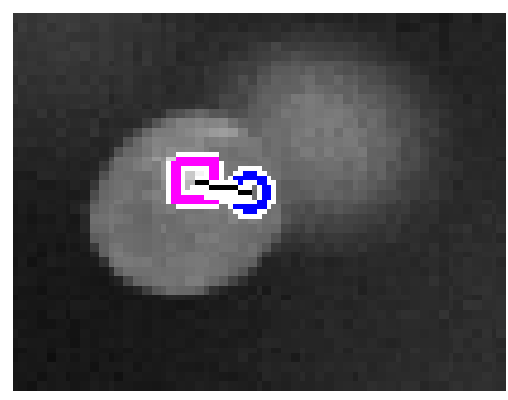

(b) $I_{9}$

Figure 4.4: Example of tracking the specular highlight of a particle. The square marks the highlights position in the current image and the circle marks its preceding location.

images $\left\{I_{1078+i}\right\}_{i=0}^{80}$. Since no other means was available to evaluate the trajectories, each was checked by hand to determine its correctness.

An example output is shown in Figure 4.5 for the pair of images $I_{1083}$ and $I_{1084}$. The circles connected to squares mark the trajectories accepted by the tracker; triangles connected to X's mark those rejected (unmatched). In the example given, cropped to show only $1 / 8$ of the images for clarity, only one accepted trajectory is incorrect (the far left trajectory in Figure 4.5(b)). However, there are 7 rejected trajectories: 3 were due to missing highlights in $I_{1084} ; 3$ were due to failures in the estimated positions, caught by the tracker; and 1 was due to the particle moving out of the imaged area. This example shows the different types of trajectories, both accepted and rejected, that have been evaluated for 
all images in $\mathcal{I}$.

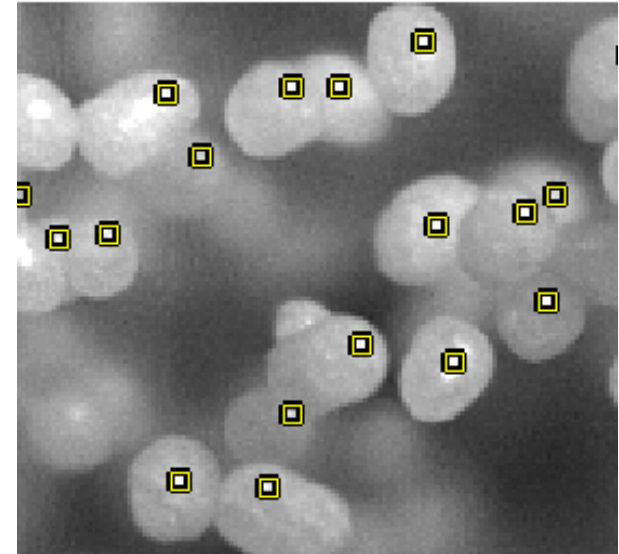

(a) $I_{1083}$

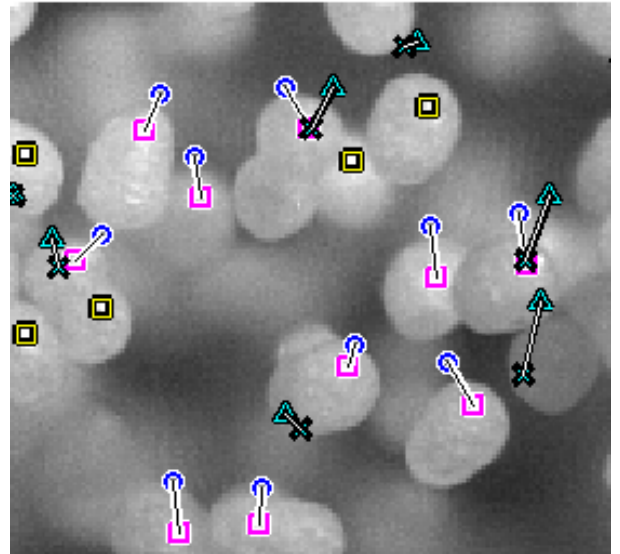

(b) $I_{1084}$

Figure 4.5: Example of tracking the specular highlights. The squares mark the highlight positions in the current image and the circles mark their preceding locations. The X's show unmatched estimated positions; triangles show the previous locations of those unmatched highlights. The images have been cropped to show only about $1 / 8$ of their total area; this has been done for clarity.

The results of the evaluation are shown in Figure 4.6 as the relative frequency of each event given the total number of detected highlights. "Accept: True" and "Accept: False" account for the trajectories output by the tracking system that were correct and incorrect, respectively. The measures prefaced by "Reject" account for those trajectories that were dismissed by the tracking system as invalid. "Missing Highlight" refers to a failure due to a matching highlight not existing in the second image; "Tracking Failure" refers to a failure borne from the tracking algorithm itself; and "Exit" refers to particles that moved out of the imaging window.

Initially the results show a large percentage of valid trajectories output by the tracker, but as the concentration increases between frames 1098 and 1013 (see Figure 3.3) the amount of valid trajectories quickly decreases. It was expected that, as the concentration decreases back to the dilute phase between frames 1123 and 1138, the valid trajectories would recover. This, however, did not occur due to the high density of background particles in that portion of the sequence. These background particles create multiple motions around the neighborhood of specular highlights, reducing the effectiveness of the local motion model. 


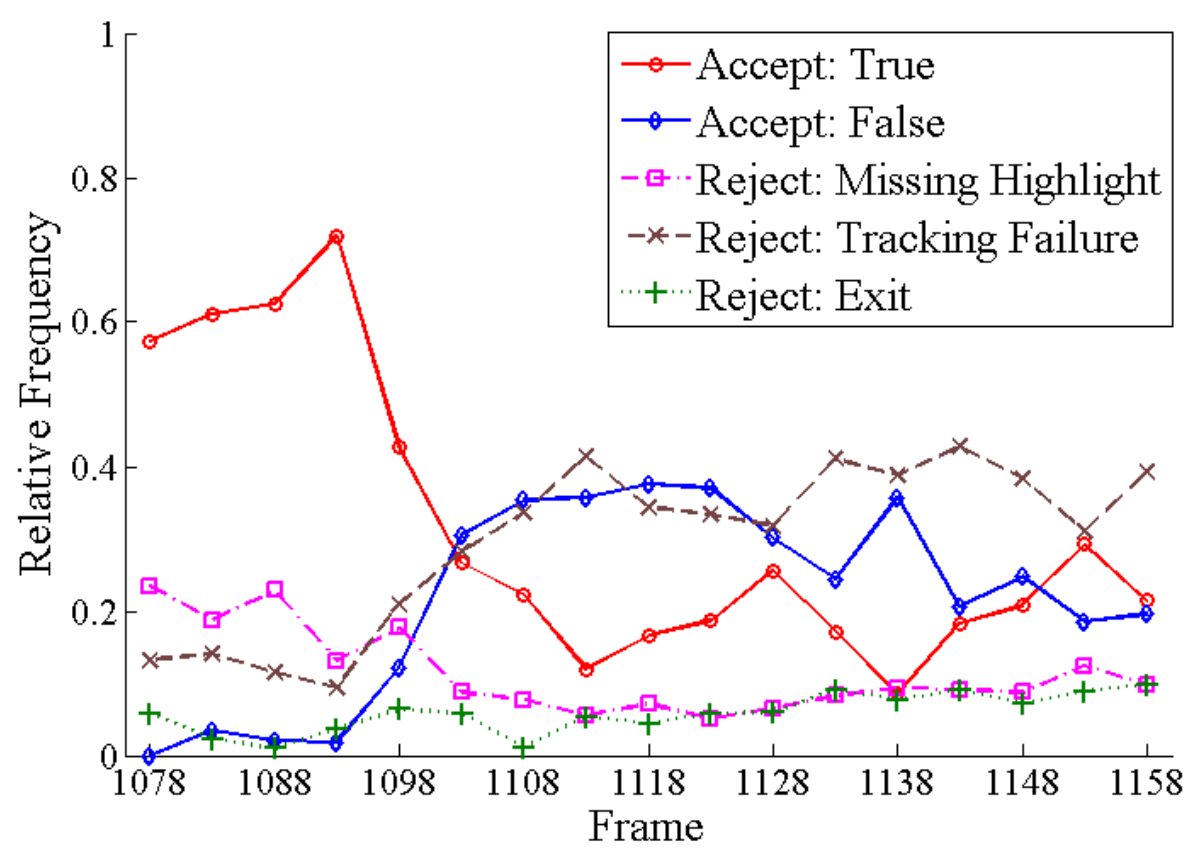

Figure 4.6: Results of tracking the specular highlights in the sequence $\mathcal{I}$. "Accept" events account for the trajectories in the output of the tracker whereas "Reject" events are those that were detected and eliminated prior to output.

\subsection{Estimated Particle Velocities}

Once the particle displacements have been determined for a pair of frames, its conversion to a measure in meters per second is straightforward. For the images in this study, 512 pixels is approximately equivalent to $16 \mathrm{~mm}$. The frame rate is known, but can vary depending on the image sequence. Typically, the images given as examples were captured at 2000 frames per second. For a displacement $d$ in pixels from one frame to the next, this translates to

$$
\frac{d \text { pixels }}{1 \text { frame }} \cdot \frac{16 \mathrm{~mm}}{512 \text { pixels }} \cdot \frac{1 \mathrm{~m}}{1000 \mathrm{~mm}} \cdot \frac{2000 \text { frames }}{1 \mathrm{~s}}=\frac{1}{16} d \mathrm{~m} / \mathrm{s}
$$

\subsection{Summary}

While showing its potential in determining concentrations and 2-dimensional velocities, specularities cannot be used to determine particle depths since they are not actually on the particle surface [10]. Also, their shape may change as the particle moves and rotates, 
or they may disappear altogether. These add complications to the process and can induce errors in the tracking algorithm. The algorithm is able to detect many of the failures, but a number of trajectories are still falsely accepted. 


\section{Chapter 5}

\section{Future Work}

\subsection{Image Preprocessing}

A potentially useful area of additional research for this project would be to develop a technique to directly suppress the out-of-focus background particles. Using the specular highlights and the high-intensity noise images to track the particles eliminated the majority of these, but suppressing the background particles first may help reduce the number of falsely detected highlights. This would serve to improve the estimated concentration as well as, potentially, the effectiveness of the tracker.

\subsection{Particle Detection}

In detecting the particles from the specular highlights, two major problems exist: (1) highlights can be detected on out-of-focus particles; and (2) large particles can have multiple highlights. Both problems overestimate the number of particles on the focal plane and can create complications for the tracking algorithm.

One possibility of reducing the problem of false highlights is to couple the highlight detection method with a pixel-wise classification scheme, possibly one of those described in §3.3. The local neighborhood of each candidate highlight could be used to determine whether the "detected" particle was actually on the focal plane.

For the second problem, a distance-based scheme could be employed to determine if highlights were closer than a single particle diameter. This of course requires knowledge of the mean particle diameter and could accidentally destroy highlights for very small particles. 
Boundary information could also be tried, although this has been shown to be unreliable.

\subsection{Particle Tracking}

A significant area of interest is in improving the tracking algorithm. As mentioned before, new or improved image preprocessing or particle detection methods could improve the effectiveness of the tracker, although improvements can be made to the tracker itself.

One of the major drawbacks of using a local optical flow model is its behavior when faced with multiple motions in a small neighborhood. This becomes problematic with higher densities, although many particles are still successfully tracked. As the concentration increases, the flow field tends to get smoother, with the motion of particles becoming more uniform. With the concentration high, it may be possible to track a group of highlights, though care must be taken given the volatility of the fluid bed. One possible modification would be to assess the concentration of regions in an image and either direct the searching algorithm using prior information of the trajectories or use a global optical flow model which would help to regularize the predicted trajectories. 


\section{References}

[1] E. H. Adelson, C. H. Anderson, J. R. Bergen, P. J. Burt, and J. M. Ogden. Pyramid methods in image processing. RCA Engineer, 29(6):33-41, 1984.

[2] R. J. Adrian. Particle-imaging techniques for experimental fluid-mechanics. Annual Review Of Fluid Mechanics, 23:261-304, 1991.

[3] J. L. Barron, D. J. Fleet, and S. S. Beauchemin. Performance of optical flow techniques. International Journal of Computer Vision, 12(1):43-77, 1994.

[4] S. S. Beauchemin and J. L. Barron. The computation of optical flow. ACM Computing Surveys, 27(3):433-466, 1995.

[5] J. R. Bergen, P. Anandan, K. J. Hanna, and R. Hingorani. Hierarchical model-based motion estimation. In 2nd European Conference on Computer Vision, pages 237-252, London, UK, 1992. Springer-Verlag.

[6] J.-Y. Bouguet. Pyramidal implementation of the Lucas-Kanade feature tracker: Description of the algorithm. Technical report, Intel Corporation, Microprocessor Research Labs, 2000.

[7] A. C. Bovik. Handbook of Image and Video Processing. Academic Press, Inc., Orlando, FL, USA, 2nd edition, 2005.

[8] S.-T. Bow. Pattern Recognition and Image Preprocessing. Marcel Dekker, Inc., New York, NY, USA, 2002.

[9] A. Bredebusch, H. Burkhardt, U. Lackermeier, and J. Werther. Application of digital image processing methods for the analysis of flow structures in circulating fluidized beds. In 8th International Symposium on Flow Visualization, 1998.

[10] G. Brelstaff and A. Blake. Geometry from specularities. In 2nd International Conference on Computer Vision, pages 297-302, Dec 1988.

[11] J. Canny. A computational approach to edge detection. IEEE Transactions on Pattern Analysis and Machine Intelligence, PAMI-8(6):679-698, Nov. 1986.

[12] V. Caselles, J. L. Lisani, J. M. Morel, and G. Sapiro. Shape preserving local histogram modification. IEEE Transactions on Image Processing, 8(2):220-230, 1999. 
[13] T. Cebeci, J. P. Shao, F. Kafyeke, and E. Laurendeau. Computational Fluid Dynamics for Engineers. Horizons Publishing Inc, New York, 2005.

[14] R. C. Chen and L. S. Fan. Particle image velocimetry for characterizing the flow structure in 3-dimensional gas-liquid-solid fluidized-beds. Chemical Engineering Science, 47(13-14):3615-3622, 1992.

[15] C. T. Crowe, M. Sommerfeld, and Y. Tsuji. Multiphase Flows with Droplets and Particles. CRC Press, Boca Raton, Fla, 1998.

[16] R. Dalejones and T. Tjahjadi. A study and modification of the local histogram equalization algorithm. Pattern Recognition, 26(9):1373-1381, 1993.

[17] R. O. Duda, P. E. Hart, and D. G. Stork. Pattern Classification. Wiley, New York, 2nd edition, 2001.

[18] M. Feistauer. Mathematical Methods in Fluid Dynamics. Wiley, New York, 1993.

[19] M. M. Fleck. Some defects in finite-difference edge finders. IEEE Transactions on Pattern Analysis and Machine Intelligence, 14(3):337-345, 1992.

[20] R. C. Gonzalez, R. E. Woods, and S. L. Eddins. Digital Image Processing using MAT$L A B$. Pearson/Prentice Hall, Upper Saddle River, NJ, 2004.

[21] J. Hatano, N. Kido, and H. Takeuchi. Microscope visualization of solid particles in circulating fluidized beds. Powder Technology, 78(2):115-119, 1994.

[22] L. Hesselink. Digital image-processing in flow visualization. Annual Review of Fluid Mechanics, 20:421-485, 1988.

[23] R. V. Hogg and J. Ledolter. Applied Statistics for Engineers and Physical Scientists. Macmillan, New York, 2nd edition, 1992.

[24] B. P. B. Hoomans. Granular dynamics of gas-solid two-phase flows. PhD dissertation, University of Twente, 2000.

[25] B. P. B. Hoomans, J. A. M. Kuipers, W. J. Briels, and W. P. M. van Swaaij. Discrete particle simulation of bubble and slug formation in a two-dimensional gas-fluidised bed: A hard-sphere approach. Chemical Engineering Science, 51(1):99-118, 1996.

[26] M. Horio. Cluster and agglomerate formation in fluidized suspensions. In 2nd International Conference on Multiphase Flow, Apr 1995.

[27] M. Horio and H. Kuroki. Three-dimensional flow visualization of dilutely dispersed solids in bubbling and circulating fluidized beds. Chemical Engineering Science, 49(15):2413-2421, 1994.

[28] B. K. P. Horn and B. G. Schunck. Determining optical flow. Artificial Intelligence, 17(1-3):185-203, 1981. 
[29] A. K. Jain, R. P. W. Duin, and J. C. Mao. Statistical pattern recognition: A review. IEEE Transactions on Pattern Analysis and Machine Intelligence, 22(1):4-37, 2000.

[30] B. R. Jennings and K. Parslow. Particle size measurement: the equivalent spherical diameter. Proceedings of the Royal Society of London, Series A-Mathematical and Physical Sciences, 419(1856):137-149, 1988.

[31] E. Krotkov. Focusing. International Journal of Computer Vision, 1(3):223-237, 1987.

[32] U. Lackermeier, C. Rudnick, J. Werther, A. Bredebusch, and H. Burkhardt. Visualization of flow structures inside a circulating fluidized bed by means of laser sheet and image processing. Powder Technology, 114(1-3):71-83, 2001.

[33] L. Lam, S.-W. Lee, and C. Y. Suen. Thinning methodologies-a comprehensive survey. IEEE Transactions on Pattern Analysis and Machine Intelligence, 14(9):869-885, Sept. 1992.

[34] A. Leon-Garcia. Probability and Random Processes For Electrical Engineering. Addison-Wesley, Reading, MA, USA, 2nd edition, 1994.

[35] H. Li, Y. Xia, Y. Tung, and M. Kwauk. Micro-visualization of clusters in a fast fluidized bed. Powder Technology, 66(3):231-235, 1991.

[36] H. Li, Q. Zhu, H. Liu, and Y. Zhou. The cluster size distribution and motion behavior in a fast fluidized bed. Powder Technology, 84:241-246, 1995.

[37] J. Li. Euler-Lagrange simulation of flow structure formation and evolution in dense gas-solid flows. PhD dissertation, University of Twente, 2003.

[38] B. D. Lucas and T. Kanade. An iterative image registration technique with an application to stereo vision. In DARPA Image Understanding Workshop, pages 121-130, April 1981.

[39] B. D. Lucas and T. Kanade. An iterative image registration technique with an application to stereo vision. In 7th International Joint Conference on Artificial Intelligence, pages 674-679, August 1981.

[40] S. Matsuda, H. Hatano, H. Takeuchi, A. T. Pyatenko, and K. Tsuchiya. Motion of individual solid particles in a circulating fluidized bed riser. In M. Kwauk and J. Li, editors, 5th International Conference on Circulating Fluidized Beds, pages 176-181. Science Press, 1996.

[41] J. S. Mei, E. R. Monazam, and L.J. Shadle. Flow regime study of a light material in an industrial scale cold flow circulating fluidized bed. Journal of Energy Resources Technology, 128(2):129-134, 2006.

[42] L. Moldavsky, A. Goldshtein, K. Shuster, M. Fichman, D. Pnueli, M. Shapiro, and C. Gutfinger. Confined fluidized bed - model and experiments. International Journal of Multiphase Flow, 31(8):957-967, 2005. 
[43] M. Oren and S. K. Nayar. A theory of specular surface geometry. In IEEE International Conference on Computer Vision, volume 0, page 740, Los Alamitos, CA, USA, 1995. IEEE Computer Society.

[44] M. Osadchy, D. Jacobs, and R. Ramamoorthi. Using specularities for recognition. In IEEE International Conference on Computer Vision, volume 2, page 1512, Los Alamitos, CA, USA, 2003. IEEE Computer Society.

[45] N. Otsu. Threshold selection method from gray-level histograms. IEEE Transactions on Systems, Man, and Cybernetics, 9(1):62-66, 1979.

[46] J. Ouyang and J. H. Li. Particle-motion-resolved discrete model for simulating gas-solid fluidization. Chemical Engineering Science, 54(13-14):2077-2083, 1999.

[47] J. Park, Y. Huang, R. Turton, P. Famouri, and E. J. Boyle. The control of bed height and solids circulation rate in the standpipe of a cold flow circulating fluidized bed. Powder Technology, 150(3):176-184, 2005.

[48] M. Rhodes, H. Mineo, and T. Hirama. Particle motion at the wall of a circulating fluidized bed. Powder Technology, 70:207-214, 1992.

[49] J. F. Richardson and J. H. Harker. Chemical Engineering: Particle Technology and Separation Processes, volume 2 of Coulson and Richardson's Chemical Engineering Series. Butterworth-Heineman, Woburn, Mass., 5th edition, 2002.

[50] C. Rudnick and J. Werther. The discrimination of cluster characteristics from fiberoptical probe signals in circulating fluidized beds. In L. S. Fan and T. M. Knowlton, editors, Fluidization IX, pages 573-580. Engineering Foundation, 1998.

[51] B. A. Saadevandi and R. Turton. The application of computer-based imaging to the measurements of particle velocity and voidage profiles in a fluidized bed. Powder Technology, 98(2):183-189, 1998.

[52] D. G. Seol, T. Bhaumik, C. Bergmann, and S. A. Socolofsky. Particle image velocimetry measurements of the mean flow characteristics in a bubble plume. Journal of Engineering Mechanics, 133(6):665-676, 2007.

[53] L. J. Shadle, J. Spenik, A. Sarra, and J. Ontko. Factorial tests on process operating conditions and bed fines on the circulating fluid bed performance. Industrial $\&$ Engineering Chemistry Research, 43(15):4166-4173, 2004.

[54] H. Shi. Experimental research of flow structure in a gas-solid circulating fluidized bed riser by PIV. Journal of Hydrodynamics, Series B, 19(6):712-719, 2007.

[55] J. E. Solem, H. Aanaes, and A. Heyden. A variational analysis of shape from specularities using sparse data. In Proceedings of the 2nd International Symposium on $3 D$ Data Processing, Visualization and Transmission, pages 26-33, Sept. 2004. 
[56] H. Takeuchi and T. Hirama. Flow visualization in the riser of a circulating fluidized bed. In P. Basu, M. Horio, and M. Hasatani, editors, Circulating Fluidized Bed Technology III, pages 177-182, 1991.

[57] H. Takeuchi, A. T. Pyatenko, and H. Hatano. Gross behavior of parabolic strands in a riser. In L. S. Fan and T. M. Knowlton, editors, Fluidization IX, pages 177-182. Engineering Foundation, 1998.

[58] V. T. Tom and G. J. Wolfe. Adaptive histogram equalization and its applications. Society of Photo-Optical Instrumentation Engineers, 359:204-209, 1982.

[59] C. Tomasi and T. Kanade. Detection and tracking of point features. Technical report cmu-cs-91-132, Carnegie Mellon University, April 1991.

[60] Z. Wang and A. C. Bovik. Mean squared error: Love it or leave it? - a new look at signal fidelity measures. IEEE Signal Processing Magazine, 26(1):98-117, Jan. 2009.

[61] J. F. Wendt, J. D. Anderson, and Von Karman Institute for Fluid Dynamics. Computational Fluid Dynamics: An Introduction. Springer, New York, 2nd edition, 1996.

[62] J. Werther. Measurement techniques in fluidized beds. Powder Technology, 102(1):15$36,1999$.

[63] J. Werther, B. Hage, and C. Rudnick. A comparison of laser doppler and single-fibre reflection probes for the measurement of the velocity of solids in a gas-solid circulating fluidized bed. Chemical Engineering and Processing, 35:381-391, 1996. 